\title{
Charge radii in covariant density functional theory: a global view
}

\author{
U. C. Perera, ${ }^{1}$ A. V. Afanasjev, ${ }^{1}$ and P. Ring ${ }^{2}$ \\ ${ }^{1}$ Department of Physics and Astronomy, Mississippi State University, MS 39762 \\ ${ }^{2}$ Fakultät für Physik, Technische Universität München, D-85748 Garching, Germany
}

(Dated: November 10, 2021)

\begin{abstract}
A systematic global investigation of differential charge radii has been performed within the CDFT framework for the first time. Theoretical results obtained with conventional covariant energy density functionals and the separable pairing interaction of Ref. [1] are compared with experimental differential charge radii in the regions of the nuclear chart in which available experimental data crosses the neutron shell closures at $N=28,50,82$ and 126. The analysis of absolute differential radii of different isotopic chains and their relative properties indicate clearly that such properties are reasonably well described in model calculations in the cases when the mean-field approximation is justified. However, while the observed clusterization of differential charge radii of different isotopic chains is well described above the $N=50$ and $N=126$ shell closures, it is more difficult to reproduce it above the $N=28$ and $N=82$ shell closures because of possible deficiencies in the underlying single-particle structure. The impact of the latter has been evaluated for spherical shapes and it was shown that the relative energies of the single-particle states and the patterns of their occupation with increasing neutron number have an appreciable impact on the evolution of the $\delta\left\langle r^{2}\right\rangle^{N, N^{\prime}}$ values. These factors also limit the predictive power of model calculations in the regions of high densities of the single-particle states of different origin. It is shown that the kinks in the charge radii at neutron shell closures are due to the underlying single-particle structure and due to weakening or collapse of pairing at these closures. The regions of the nuclear chart in which the correlations beyond mean-field are expected to have an impact on charge radii are indicated; the analysis shows that the assignment of a calculated excited prolate minimum to the experimental ground state allows to understand the trends of the evolution of differential charge radii with neutron number in many cases of shape coexistence even at the mean-field level. It is usually assumed that pairing is a dominant contributor to odd-even staggering (OES) in charge radii. Our analysis paints a more complicated picture. It suggests a new mechanism in which the fragmentation of the single-particle content of the ground state in odd-mass nuclei due to particle-vibration coupling provides a significant contribution to OES in charge radii.
\end{abstract}

\section{INTRODUCTION}

Together with nuclear masses, the charge radii are among the most fundamental properties of atomic nuclei. The essential information on the saturation density of symmetric nuclear matter is imprinted into them. They also depend on the properties of nuclear forces and nuclear many-body dynamics.

Significant experimental efforts have been dedicated over the decades for the measurement of charge radii $[2,3]$. While the changes of the charge radii within the isotopic chain are measured with high precision using laser spectroscopy, the situation with the measurements of absolute values of root-mean-square $(\mathrm{rms})$ charge radii $r_{c h}$ is less satisfactory because of lower precision of their determination in muonic spectra and electronic scattering experiments and the impossibility of such experiments in radioactive elements [2,3]. For example, for nuclei with proton number $Z>83$, (uranium is the exception), there are no experimental data for the absolute nuclear charge radii [2]. Theoretical calculations within different density functional theories (DFTs) provide a quite accurate global description of experimental charge radii presented in the compilation of Ref. [2]: the rms deviations of calculated $r_{c h}$ from experimental ones are at the level of $\approx 0.03 \mathrm{fm}[4]$. Considering that the average experimental $\mathrm{rms}$ charge radius in the nuclear chart is around $4.8 \mathrm{fm}$ (see, for example, Fig. 23 in Ref. [4] and Figs. 2-4 in Ref. [2]), this amounts to high average precision of $0.625 \%$ in the prediction of charge radii. However, this information has to be taken with a grain of salt because of the issues mentioned above with the measurements of absolute values of rms charge radii and some reliance on interpolation/extrapolation procedures in the compilation of Ref. [2].

Thus, the differential mean-square (ms) charge radii (see Eq. (6) below for definition), measured with high precision within the isotopic chains, become an important quantity. The evolution of the charge radii within the isotopic chain with increasing neutron number is defined by the pull on the proton states generated by neutrons gradually added to the nuclear system. This is, in reality, a quite complicated and, in some cases, contraintuitive process. Here the strong nuclear symmetry energy acts to increase the overlap between all the proton states and the overall nuclear density. This effect is expected to be enhanced when the overlap between the wave functions is maximal. The most investigated case here is the kink in charge radii at $N=126$ and the evolution of charge radii above $N=126$ in the $\mathrm{Pb}$ isotopic chain. The pattern of these effects critically depends on the occupation of the $2 g_{9 / 2}$ and $1 i_{11 / 2}$ orbitals, on their relative energies, and on how close they are in energy 
$[5,6]$. Both in relativistic and non-relativistic density functional theories (DFT), the single-particle rms neutron radius of the $2 g_{9 / 2}$ orbital is larger than that of the $1 i_{11 / 2}$ orbital (by $\approx 0.6 \mathrm{fm}$ [see Table II below] and $\approx 1.0$ fm [see Ref. [5]], respectively). Intuitively (for example, by using liquid drop model concepts), the occupation of the neutron $2 g_{9 / 2}$ orbital would bring a larger proton radius as compared with the occupation of the $1 i_{11 / 2}$ orbital. However, the DFT calculations showed an opposite trend $[5,6]$ the deep microscopic origin of which has been found only in Ref. [6]. It is traced back to a nodal structure $^{1}$ of these two orbitals $\left(n=1\right.$ for $1 i_{11 / 2}$ and $n=2$ for $2 g_{9 / 2}$, where $n$ stands for principal quantum number; see also Fig. 6.2 in Ref. [9] for a pattern of respective wavefunctions). The principal quantum number of the neutron $1 i_{11 / 2}$ orbital is the same as for the majority of occupied proton orbitals (including deeply bound ones). This leads to a large overlap of their wave functions and thus provides a large pull of these neutron states on proton orbitals via the symmetry energy and allows the reproduction of the kink in charge radii at $N=126$.

A significant amount of experimental data on charge radii has been collected over the years: the review [2] provides a compilation of such data measured by early $2011^{2}$. In recent years an explosion of high-quality measurements of charge radii is observed (see, for example, review [3]). They cover, for example, in recent years the $\mathrm{K}$ [10], Ca [11, 12], Cu [13], Cd [14], Sn [15, 16], Hg [17], Bi [18], At [19], Ac [20] and No [21] isotopic chains. These experimental studies are supplemented mostly by a theoretical analysis within non-relativistic Skyrme or Fayans DFTs (see overview below) and occasionally by the analysis within Gogny DFT (the Ca isotopes in Ref. [11]), the CDFT (the $\mathrm{Pb}$ and $\mathrm{Hg}$ isotopes in Ref. [17] and No isotopes in Ref. [22]) or non-relativistic ab-initio calculations (see Refs. $[10,11]$ ).

They reveal several interesting features. The most familiar are the kinks in charge radii at neutron numbers corresponding to shell closures and the odd-even staggering (OES) in charge radii. In charge radii, a shell closure is observed as a sudden increase in the rate of the change of charge radius of the isotopes just beyond magic shell closure; this leads to the kinks in charge radii which are well known at $N=28,50,82$ and $126[2,11,15,23]$. In addition, the analysis of experimental data presented

\footnotetext{
${ }^{1}$ Note that the nodal structure of the wavefunctions plays an important role not only in the evolution of charge radii of spherical nuclei. In extremely deformed nuclei, it also defines the necessary conditions for $\alpha$ clusterization in very light nuclei, and its suppression with the increase of mass number [7]. Moreover, the nodal structure of the deformed wavefunctions allows us to understand the coexistence of ellipsoidal mean-field-type structures and nuclear molecules at similar excitation energies and the features of particle-hole excitations connecting these two types of structures $[7,8]$.

2 The experimental data shown in the present paper is based on this compilation supplemented when available with more recent data.
}

in Ref. [23] reveals a puzzling feature related to similar slopes of differential charge radii $\delta\left\langle r^{2}\right\rangle$ as a function of neutron number above the neutron shell closures for different isotopic chains (see Fig. 5 in Ref. [23] for evenand odd- $Z$ isotopic chains and Figs. 12(a), 17(a), 19(a) and 25 (a) below for only even- $Z$ isotope chains). On the contrary, all these isotopic chains have different slopes of differential charge radii for neutron numbers below the shell closures. To our knowledge, this observed feature, which exists in the $\mathrm{Ca}, \mathrm{Sr}, \mathrm{Sn}$, and $\mathrm{Pb}$ regions, has not been addressed in a systematic theoretical analysis so far.

Different theoretical approaches have been used with different degrees of success to describe the evolution of charge radii in various isotopic chains. The initial focus of such studies was the kink in differential charge radii of the $\mathrm{Pb}$ isotopes at $N=126$ but later studies covered also other isotopic chains. The calculations in non-relativistic density functional theories (NR-DFTs) based on conventional energy density functionals (EDFs) were unable to reproduce the kink in the $\mathrm{Pb}$ isotopes $[15,24]$. On the contrary, this kink is quite successfully reproduced in covariant DFT (CDFT) for all employed covariant energy density functionals (CEDFs) $[17,25,26]$. As discussed above the relative energies of the $1 i_{11 / 2}$ and $2 g_{9 / 2}$ neutron orbitals play a crucial role in this difference between NR-DFT and CDFT results in the $\mathrm{Pb}$ isotopes. Two possible ways of resolving the problem emerged in the NR-DFTs. The first one includes a modification of spinorbit interaction either by its fitting to CDFT results [5] or by introducing a density dependence in the spinorbit interaction [27]. The second approach, introduced by Fayans et al. [28-30] includes an extension of nonrelativistic functionals by adding gradient terms into the surface part and the pairing interaction. Although this approach has been reasonably successful (especially after fitting the Fayans functionals to differential charge radii data in Ref. [31]) in the description of the evolution of charge radii [11, 13-15, 29-31], the microscopic origin of these gradient terms is not clear. It was stated that the pairing functional of the Fayans model is supposed to effectively account for the coupling to surface vibrations [31]. However, this contradicts to the observation that such a coupling is quite small for the ground states in even-even nuclei in the $\mathrm{Sn}$ and $\mathrm{Pb}$ regions (see discussion in Sec. IX below) so that the charge radii of such nuclei are not expected to be strongly modified by it.

Alternative DFT approaches are based either on non-relativistic finite range Gogny functionals, a nonrelativistic Yukawa interaction or on CEDFs. The Gogny DFT studies of differential charge radii are less frequent than those based either on the Skyrme or Fayans EDFs. For example, the isotopic evolution of charge radii in even-even and even-odd $\mathrm{Sr}, \mathrm{Zr}$, and Mo isotopes with $N=47-68$ and the impact of triaxiality on charge radii of even-even Mo isotopes with $N=62,64$, and 66 have been investigated in Ref. [32]. The differential charge radii of the ${ }^{52,48} \mathrm{Ca}$ isotopes were studied at and beyond mean-field level with the Gogny functional 
D1S in Ref. [11]. The non-relativistic HFB approach with a finite-range Yukawa interaction [33] and densitydependent spin-orbit interaction has been successfully applied for the description of differential charge radii in spherical nuclei of the $\mathrm{Ca}, \mathrm{Ni}, \mathrm{Sn}$ and $\mathrm{Pb}$ isotopic chains $[27,34,35]$.

The studies of differential charge radii are also rare within the $\mathrm{CDFT}^{3}$. The first-ever [among any type of DFT] successful description of the kink in charge radii of the lead isotopes has been achieved in Ref. [25] for CDFT with the NL-SH and NL1 functionals. This work was followed by a number of the studies of differential charge radii in spherical even-even nuclei in the $\mathrm{Ca}, \mathrm{Sn}$, and $\mathrm{Pb}$ isotopic chains in Refs. [5, 38, 39]. The odd-even staggering and the kink in charge radii of the $\mathrm{Pb}$ and $\mathrm{Hg}$ isotopes has been successfully described recently in Ref. [17] using the DD-ME2 CEDF. An ansatz for charge radii in CDFT has been suggested in Ref. [40]; it adds the phenomenological term $a_{0} / \sqrt{A}\left|\sum_{k>0}^{n} u_{k} v_{k}-\sum_{k>0}^{p} u_{k} v_{k}\right|$ to the definition of charge radii without affecting the definitions of other physical observables. Although it can describe the charge radii and their OES in selected isotopic chains, its physical meaning is not clear and it does not appear in conventional DFTs.

The goal of the present paper is to perform detailed studies of differential charge radii within the CDFT framework in order to understand specific facts, such as the underlying single-particle structure and the role of pairing, affecting the theoretical description of the evolution of charge radii with the neutron number, the presence and magnitude of the kinks and OES in the isotopic chains. Note that we employ conventional functionals which do not use any new fit parameters nor the above mentioned modifications. The aim is to understand to which extent they can provide a satisfactory description of differential charge radii in the regions of the nuclear chart in which available experimental data crosses shell closures at $N=28,50,82$ and 126 . One should note that conventional relativistic functionals provide, amongst many other nuclear properties, a reasonable description of rotational bands, which are sensitive to pairing, not only in even-even but also in odd- $A$ nuclei (see Refs. [41, 42]). Thus, they provide access to OES in the moments of inertia, the physical mechanism of which [blocking in odd- $A$ nuclei] is similar to the one partially responsible for OES in charge radii and in binding energies. At present, it is not clear whether the inclusion of gradient terms into the pairing functional will preserve

\footnotetext{
3 This is despite the fact that global studies of charge radii of eveneven nuclei located between the two-proton and two-neutron drip lines with the assessment of systematic theoretical uncertainties have been performed in Refs. $[4,36]$ and tabulated values of charge radii are publicly available in the supplemental material for Ref. [4] (for the DD-PC1 functional) and at MassExplorer [37] (for the DD-PC1, DD-ME2, DD-ME $\delta$ and NL3* CEDFs).
}

this feature. An additional goal of the present paper is to search for alternative physical mechanisms affecting differential charge radii and OES.

The paper is organized as follows. A brief outline of the theory is given in Sec. II. Charge radii and related indicators are discussed in Sec. III. The charge radii of the $\mathrm{Pb}$ isotopes are used in Sec. IV as a testing ground to evaluate the importance of the underlying single-particle structure and pairing in the evolution of differential charge radii between the two-proton and twoneutron drip lines, in the appearance of the kinks at shell closures and for the comparison of the results of calculations with and without pairing. Charge radii, their evolution with neutron number, the sources of the discrepancies between theory and experiment, absolute and relative properties of differential charge radii in different isotopic chains in the $\mathrm{Pb}, \mathrm{Sn} / \mathrm{Gd}, \mathrm{Sr}$ and $\mathrm{Ca}$ regions are discussed in detail in Secs. V, VI, VII and VIII, respectively. Note that the discussion in these sections is restricted to even-even nuclei. Sec. IX is dedicated to the analysis of odd-even staggering in charge radii and its origin. A new mechanism of OES, relying on the fragmentation of the single-particle states in odd- $N$ nuclei due to particle-vibration coupling, is suggested for the first time in this section. Finally, Sec. X summarizes the results of our paper.

\section{THEORETICAL FRAMEWORK}

In the present paper, the relativistic HartreeBogoliubov (RHB) approach is used in the calculations. The formalism of this approach is discussed in detail in Refs. $[4,43]$. The calculations are performed with computer codes that preserve either spherical or axial symmetry. The former code has been considerably modified to allow for fully self-consistent calculations of the ground and excited states in odd- $A$ spherical nuclei; in was applied for the first time in Ref. [17]. The axial code employed in Ref. [4] has been used here for calculations which include deformation.

To assess the dependence of the results on the underlying single-particle structure, several state-of-the-art covariant energy density functionals (CEDFs) such as NL3* [44], DD-PC1 [45], DD-ME2 [46], DD-ME $\delta$ [47] and PCPK1 [48] are used in the present paper. They represent the major classes of CEDFs and their global performance in describing ground state properties such as masses and charge radii of even-even nuclei has been tested in Refs. $[4,36,49]$. Note that many of the results on charge radii and the deformations of the ground states employed in the present analysis are taken from Refs. [4, 37]. This allows us to test the predictive power of the models with respect to the description of differential charge radii.

The separable pairing interaction of finite range, introduced as a simplification of the Gogny pairing by Tian et al in Ref. [1], is used in the present paper. Its matrix 
elements in $r$-space have the form

$$
\begin{aligned}
& V\left(\boldsymbol{r}_{1}, \boldsymbol{r}_{2}, \boldsymbol{r}_{1}^{\prime}, \boldsymbol{r}_{2}^{\prime}\right)= \\
& \quad=-f G \delta\left(\boldsymbol{R}-\boldsymbol{R}^{\prime}\right) P(r) P\left(r^{\prime}\right) \frac{1}{2}\left(1-P^{\sigma}\right)
\end{aligned}
$$

with $\boldsymbol{R}=\left(\boldsymbol{r}_{1}+\boldsymbol{r}_{2}\right) / 2$ and $\boldsymbol{r}=\boldsymbol{r}_{1}-\boldsymbol{r}_{2}$ being the center of mass and relative coordinates. The form factor $P(r)$ is of Gaussian shape

$$
P(r)=\frac{1}{\left(4 \pi a^{2}\right)^{3 / 2}} e^{-r^{2} / 4 a^{2}}
$$

The parameters $G=728 \mathrm{MeV} \mathrm{fm}{ }^{3}$ and $a=0.644 \mathrm{fm}$ of this interaction, which are the same for protons and neutrons, have been derived by a mapping of the ${ }^{1} \mathrm{~S}_{0}$ pairing gap of infinite nuclear matter to that of the Gogny force D1S [1] under the condition that $f=1.0$. The particle number dependence of the scaling factor $f$ of the pairing force is taken from Ref. [4].

The proton quadrupole deformation $\beta_{2}$ is defined from proton quadrupole moment $Q_{20}$ as

$$
\beta_{2}=\frac{\sqrt{5 \pi} Q_{20}}{3 Z R_{0}^{2}}
$$

where

$$
Q_{20}=\int d^{3} r \rho(\boldsymbol{r})\left(2 z^{2}-r_{\perp}^{2}\right)
$$

with $r_{\perp}^{2}=x^{2}+y^{2}$. Here $R_{0}=1.2 A^{1 / 3}$ and $\rho(\boldsymbol{r})$ stands for proton density. Eq. (3) is used also in the extraction of experimental $\beta_{2}$ deformation parameters from measured data [50]. This justifies its application despite the fact that this simple linear expression ignores the contributions of higher power/multipolarity deformations to the proton quadrupole moment. Including higher powers of $\beta_{2}$, as in Ref. [51], yields values of $\beta_{2}$ that are $\approx 10 \%$ lower.

\section{CHARGE RADII AND RELATED INDICATORS}

The charge radii were calculated from the corresponding point proton radii as

$$
r_{c h}=\sqrt{<r^{2}>_{p}+0.64} \mathrm{fm}
$$

where $<r^{2}>_{p}$ stands for proton mean square point radius and the factor 0.64 accounts for the finite-size effects of the proton. Here we have neglected the small contributions to the charge radius originating from the electric neutron form factor and the electromagnetic spin-orbit coupling $[52,53]$ as well as the corrections due to the center of mass motion. Note that the functional DDPC1 [45] has been adjusted only to nuclear binding energies.

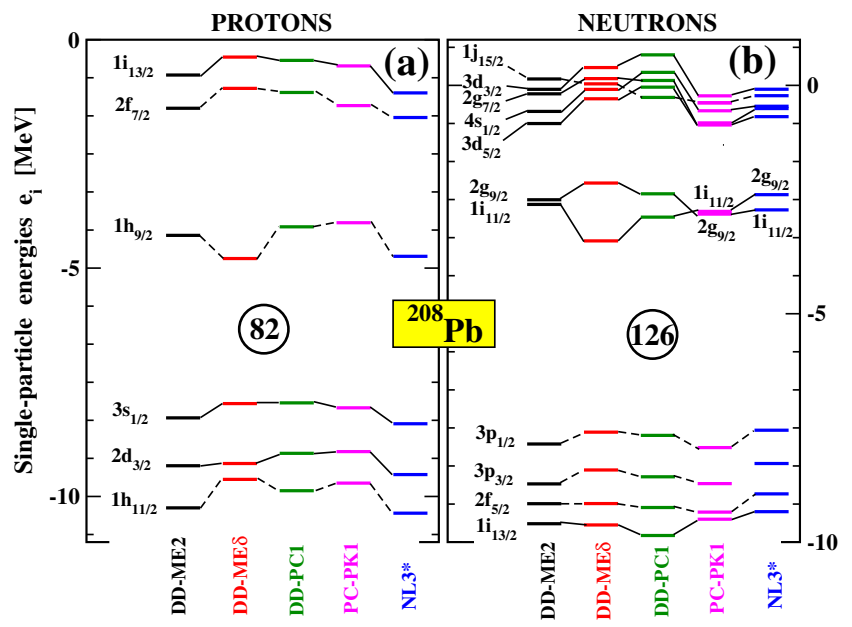

FIG. 1. Neutron and proton single-particle states at spherical shape in ${ }^{208} \mathrm{~Pb}$ obtained in the calculations without pairing with the indicated CEDFs. Solid and dashed connecting lines are used for positive- and negative-parity states. Spherical gaps are indicated.

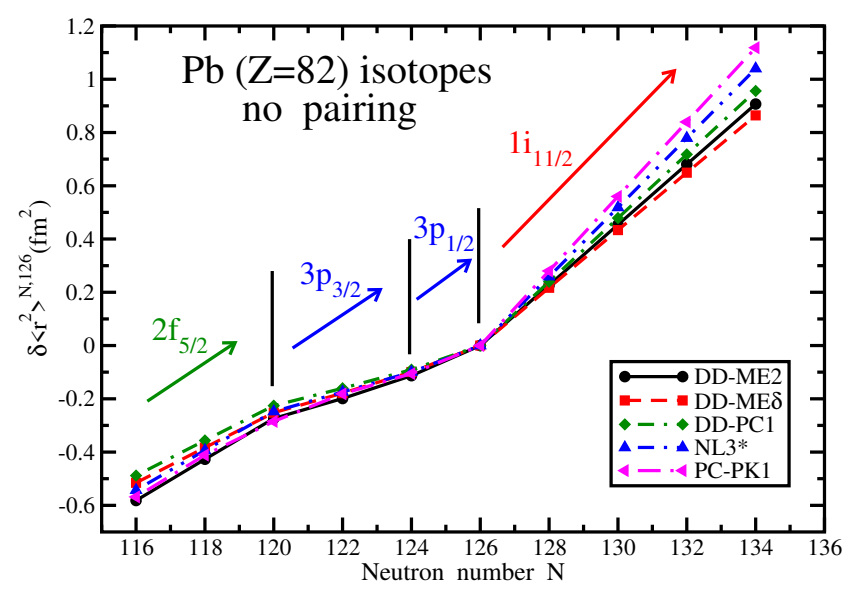

FIG. 2. The $\delta\left\langle r^{2}\right\rangle^{N, 126}$ values of the $\mathrm{Pb}$ isotopes relatively to ${ }^{208} \mathrm{~Pb}$ obtained in the calculations without pairing with the indicated CEDFs. Vertical black lines indicate the neutron numbers at which the slope of the $\delta\left\langle r^{2}\right\rangle^{N, N^{\prime}}$ curves change. The spherical subshell labels indicate the orbitals which are populated with increasing neutron number between these vertical lines (see text for details). Note that the presentation is restricted to the range of the nuclei in which experimental ground states of the $\mathrm{Pb}$ isotopes are spherical [17].

In addition, two differential indicators are commonly used to facilitate the quantitative comparison of the experimental results with those from theoretical calculations. One of them is the differential mean-square charge 
$\operatorname{radius}^{4}$

$$
\begin{aligned}
\delta\left\langle r^{2}\right\rangle_{p}^{N, N^{\prime}}= & \left\langle r^{2}\right\rangle_{p}(N)-\left\langle r^{2}\right\rangle_{p}\left(N^{\prime}\right)= \\
& =r_{c h}^{2}(N)-r_{c h}^{2}\left(N^{\prime}\right)
\end{aligned}
$$

where $N^{\prime}$ is the neutron number of the reference nucleus. Another is the three-point indicator

$$
\begin{aligned}
\Delta\left\langle r^{2}\right\rangle^{(3)}(N) & = \\
& =\frac{1}{2}\left[\left\langle r^{2}(N-1)\right\rangle+\left\langle r^{2}(N+1)\right\rangle-2\left\langle r^{2}(N)\right\rangle\right]= \\
& =\frac{1}{2}\left[r_{c h}^{2}(N-1)+r_{c h}^{2}(N+1)-2 r_{c h}^{2}(N)\right]
\end{aligned}
$$

which quantifies OES in charge radii.

In addition, the neutron skin thickness is commonly defined as the difference of proton and neutron root-meansquare (rms) radii

$$
r_{\text {skin }}=<r_{n}^{2}>^{1 / 2}-<r_{p}^{2}>^{1 / 2} .
$$

The neutron skin thickness is an important indicator of isovector properties.

\section{THE PB ISOTOPES: FROM UNPAIRED TO PAIRED RESULTS}

For a better understanding of the physical features which affect the description of charge radii it is very illustrative to start from the analysis of the results of the calculations performed without pairing but with different CEDFs representing the major classes of the CDFT models. They provide comparable global descriptions of the ground state properties $[4,49,54]$ but reveal visible differences in the single-particle properties (see, for example, Fig. 1). The addition of pairing will reveal how it affects the detailed properties of differential charge radii.

The experimental absolute value of the charge radius of the nucleus ${ }^{208} \mathrm{~Pb}$ is well described by the employed functionals (see Table I), but there exist some uncertainties in the prediction of the neutron skin in the model calculations and in its experimental measurements. One can see that non-PREX ${ }^{5}$ experiments provide neutron skins

\footnotetext{
4 This quantity is frequently written as a function of mass number $A$. However, we prefer to define it as a function of neutron number $N$ since this allows to see the behavior of the $\delta\left\langle r^{2}\right\rangle_{p}^{N, N^{\prime}}$ curves at neutron shell closures.

${ }^{5}$ Different types of non-PREX experiments are discussed in Refs. $[55,56]$ and references quoted therein. Note that the experimental data on the neutron skin are extracted in a modeldependent way in all these experiments. For instance, the neutron skin thicknesses $r_{\text {skin }}=0.161 \pm 0.042 \mathrm{fm}$ [57] and $r_{\text {skin }}=0.190 \pm 0.028 \mathrm{fm}[58]$ obtained from the energy of the antianalog giant dipole resonance rely on relativistic proton-neutron quasiparticle random-phase approximation calculations based on the RHB model. Another example is the value of the neutron skin thickness of $r_{\text {skin }}=0.15 \pm 0.03$ (stat) ${ }_{-0.03}^{+0.01}$ (sys) fm extracted
}

TABLE I. The charge radius $r_{c h}$ and the neutron skin $r_{s k i n}$ of the nucleus ${ }^{208} \mathrm{~Pb}$ obtained in calculations with the indicated functionals. The experimental value of $r_{c h}$ is taken from Ref. [2]. Two experimental values are provided for $r_{\text {skin }}$ : one (approximate, labelled as non-PREX) obtained from the experiments which do not employ parity violating electron scattering on nuclei (PREX) (see discussion in Sect. X of Ref. [4] for more details) and another (labelled as PREX-II) from the PREX-II experiment [61].

\begin{tabular}{l|c|c}
\hline \hline CEDF & $r_{c h}[\mathrm{fm}]$ & $r_{\text {skin }}[\mathrm{fm}]$ \\
\hline DDME2 & 5.518 & 0.193 \\
DDME $\delta$ & 5.509 & 0.186 \\
DD-PC1 & 5.513 & 0.202 \\
NL3* & 5.509 & 0.288 \\
PCPK1 & 5.519 & 0.257 \\
exper. & $5.5012 \pm 0.0013$ & $\approx 0.19[$ non-PREX] \\
& & $0.283 \pm 0.071[\mathrm{PREX}-\mathrm{II}]$ \\
\hline \hline
\end{tabular}

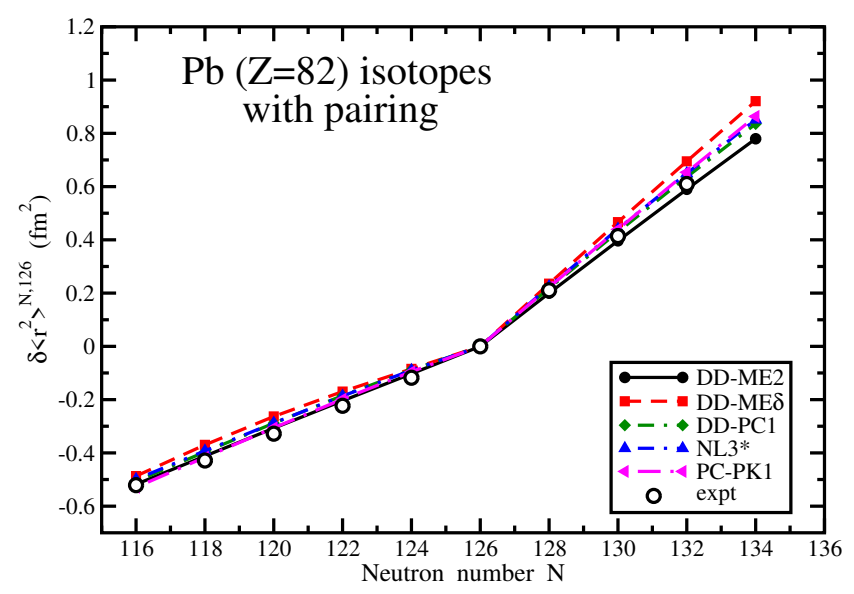

FIG. 3. The same as Fig. 2 but for the results of calculations with pairing included. The experimental data are taken from Ref. [17].

which are by $\approx 0.09 \mathrm{fm}$ smaller than the one obtained in the PREX-II experiment.

The $\delta\left\langle r^{2}\right\rangle^{N, 126}$ values of the $\mathrm{Pb}$ isotopes obtained with various CEDFs in calculations without pairing are shown in Fig. 2. One can see that the slope of this function (namely, the derivative $\delta\left\langle r^{2}\right\rangle^{N, 126} / \delta N$ ) changes at $N=120, N=124$ and $N=126$ in all functionals. The changes in the slope of $\delta\left\langle r^{2}\right\rangle^{N, 126}$ as a function of $N$ are traced back to the changes in the occupation of different spherical neutron subshells. The sequence of the

from coherent pion photoproduction cross sections [59]. In this case the extraction of information on the nucleon density distribution depends on the comparison of the measured $\left(\gamma, \pi^{0}\right)$ cross sections with model calculations. On the contrary, the electroweak probe (PREX types of experiment) has the advantage over experiments using hadronic probes that it allows a nearly model-independent extraction of the neutron radius that is independent of most strong interaction uncertainties [60]. 


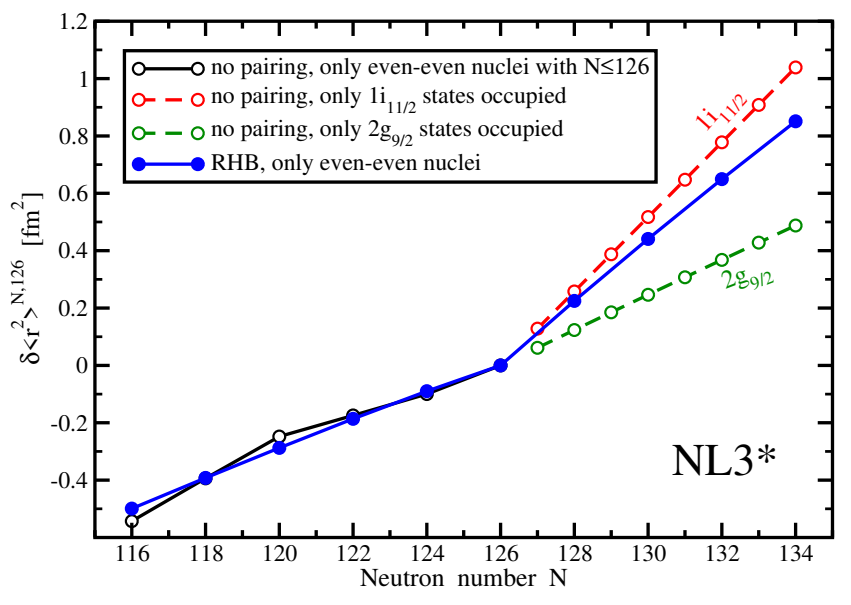

FIG. 4. The $\delta\left\langle r^{2}\right\rangle^{N, 126}$ values of $\mathrm{Pb}$ isotopes relatively to ${ }^{208} \mathrm{~Pb}$ obtained in calculations without pairing with the NL3* CEDF (see text for details). The results of RHB calculations with pairing for even-even nuclei are presented for comparison.

occupation of different spherical subshells with increasing neutron number is the same for all functionals (see Figs. 2 and 1). The $\nu 2 f_{5 / 2}$ subshell is occupied for neutron numbers $N=115-120^{6}$. For higher neutron numbers $N=121-124$, the $\nu 3 p_{3 / 2}$ subshell gradually fills up with increasing neutron number. This change of the occupation from the $\nu 2 f_{5 / 2}$ to the $\nu 3 p_{3 / 2}$ subshell leads to the change of the slope of the $\delta\left\langle r^{2}\right\rangle^{N, 126}$ function at $N=120$ because these two subshells have different density distributions and thus different neutron radii. The next change of the slope of this function takes place at $N=124$ at the transition from the occupation of the $\nu 3 p_{3 / 2}$ to the $\nu 3 p_{1 / 2}$ subshell. Since these two subshells are spin-orbit partners, they have the same orbital quantum number and, as a consequence, very similar spatial distributions of the density. Minor differences in the latter are caused by the fact that the $\nu 3 p_{1 / 2}$ subshell is located somewhat higher in energy than the $\nu 3 p_{3 / 2}$ one [see Fig. 1(b)] and thus in the region of a somewhat larger radius of the nucleonic potential.

The inclusion of pairing modifies the results visibly as compared to those obtained in the calculations without pairing (compare Fig. 3 with Fig. 2). First, the changes of the slopes of the $\delta\left\langle r^{2}\right\rangle^{N, 126}$ curves at $N=120$ and $N=124$, which are present in the calculations without pairing, are almost washed out when pairing is taken into account. This is because pairing modifies the occupation of different subshells (see, for example, Fig. 5) and

\footnotetext{
${ }^{6}$ In the calculations without pairing, the occupation of either an odd neutron (in odd- $A$ nuclei) or a pair of neutrons (in even-even nuclei) from the same spherical subshell leads to the same slope of the $\delta\left\langle r^{2}\right\rangle^{N, 126}$ function. Thus, for simplicity we consider only even-even nuclei in this part of the discussion.
}

thus the evolution of the $\delta\left\langle r^{2}\right\rangle^{N, 126}$ values with neutron number becomes more gradual at these particle numbers. Second, the kink in the $\delta\left\langle r^{2}\right\rangle^{N, 126}$ values at $N=126$ still exists because of the large shell closure at this particle number which leads to the collapse of pairing. If (hypothetically) pairing would survive at $N=126$, the kink would be less pronounced. Third, the spreads and absolute magnitudes in the predictions of the $\delta\left\langle r^{2}\right\rangle^{116,126}$ and $\delta\left\langle r^{2}\right\rangle^{134,126}$ values (the values taken at the extremes of the plots presented in Figs. 2 and 3 ) are reduced when the pairing is taken into account. These values are located in the ranges from $-0.58 \mathrm{fm}^{2}$ to $-0.48 \mathrm{fm}^{2}$ (from $-0.51 \mathrm{fm}^{2}$ to $-0.49 \mathrm{fm}^{2}$ ) and from $0.86 \mathrm{fm}^{2}$ to $1.12 \mathrm{fm}^{2}$ (from $0.78 \mathrm{fm}^{2}$ to $0.92 \mathrm{fm}^{2}$ ) in the calculations without (with) pairing, respectively. Fourth, the relative order of the results obtained with different functionals in Figs. 2 and 3 at given neutron number is different in the calculations with and without pairing. This is best illustrated by considering the case of $N=134$ and the sequence of functionals ordered according to the increase of calculated $\delta\left\langle r^{2}\right\rangle^{N, 126}$ values. The sequences of functionals are DD-ME $\delta$, DD-ME2, DD-PC1, NL3* and PC-PK1 in the calculations without pairing (see Fig. 4) and DD-ME2, DD-PC1, NL3*, PC-PK1 and DD-ME $\delta$ in the calculations with pairing (see Fig. 3).

To better understand these features we analyze the results of the calculations presented in Figs. 4 and 5 . The calculations without pairing clearly show that the occupation of the $\nu 1 i_{11 / 2}$ subshell for $N>126$ is needed for the formation of the experimentally observed kink at $N=126$ and that the occupation of the $\nu 2 g_{9 / 2}$ subshell above $\mathrm{N}=126$ does not lead to the formation of a kink at $N=126$ (see Fig. 4). The inclusion of pairing leads to a partial occupation of these two subshells (see Fig. 5 ) and thus to $\delta\left\langle r^{2}\right\rangle^{N, 126}$ values located in between of those obtained in the calculations without pairing for the occupation of these two subshells (see Fig. 4).

Fig. 4 also illustrates that the sequential occupation of a given subshell (either $\nu 1 i_{11 / 2}$ or $\nu 2 g_{9 / 2}$ ) above $N=126$ in odd- $A$ and even-even nuclei leads to $\delta\left\langle r^{2}\right\rangle^{N, 126}$ values that form a straight line as a function of neutron number. Thus, with this occupation pattern, the OES of charge radii cannot be formed in the calculations without pairing. However, as discussed in detail in Ref. [17] the scattering of the occupation of the orbitals in these subshells will lead to the formation of an OES in the charge radii which has a magnitude comparable to experiment.

Fig. 5 allows to better understand the role of pairing and the impact of the underlying single-particle structure on the magnitude of the kink in the charge radii at $N=126$. This figure focuses on the occupation pattern and the relative energies of the neutron $2 g_{9 / 2}$ and $1 i_{11 / 2}$ orbitals located above $N=126$. Other states (such as $3 d_{5 / 2}, 4 s_{1 / 2}, 2 g_{7 / 2}$ etc) do not play a significant role in the creation of the kink since they are separated by a large energy gap from the pair of the states under study (see Fig. 1(b)) and their occupation in the presence of 
pairing is small. The occupation probability $v^{2} /(2 j+1)$ of the respective subshell is defined in such a way that it is equal to 1 or 0 when a given subshell of multiplicity $2 j+1$ is either fully occupied or empty. This occupation probability grows almost linearly with increasing neutron number [see Fig. 5(a)].

The largest energy gap between the neutron $2 g_{9 / 2}$ and $1 i_{11 / 2}$ subshells exists in the DD-ME $\delta$ functional for all neutron numbers of interest (see Fig. 5(b)). As a consequence, for a given neutron number the occupation of the lowest (highest) in energy $1 i_{11 / 2}\left(2 g_{9 / 2}\right)$ subshell is large (small) but they gradually raise with increasing neutron number (see Fig. 5(a)). This significant preference in the occupation of the $1 i_{11 / 2}$ subshell leads to the largest $\delta\left\langle r^{2}\right\rangle^{N, 126} / \delta N$ values for $N>126$ isotopes among the considered functionals which exceeds the experimental data (see Fig. 3). In all other functionals, the gap between the $2 g_{9 / 2}$ and $1 i_{11 / 2}$ subshells is smaller (see Fig. $5(\mathrm{~b}))$ but still the occupation of the $1 i_{11 / 2}$ subshell is favored $^{7}$. Thus, as compared with the DD-ME $\delta$ functional the difference in the occupation of these orbitals becomes smaller [see Fig. 5(a)]. This leads to a reduction of the $\delta\left\langle r^{2}\right\rangle^{N, 126} / \delta N$ values for $N>126$ nuclei which now become close to experiment (see Fig. 3).

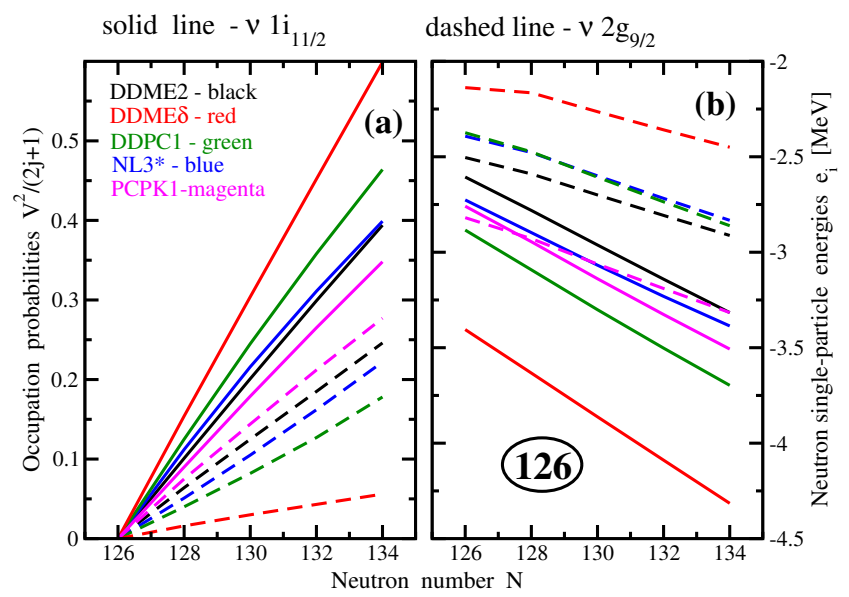

FIG. 5. (a) The evolution of occupation probabilities $v^{2} /(2 j+$ 1) of the neutron $2 g_{9 / 2}$ and $1 i_{11 / 2}$ orbitals as a function of neutron number in the $N>126$ nuclei. (b) The evolution of the energies of these single-particle states as a function of neutron number.

There are large similarities between the results obtained with the different CEDFs presented in Fig. 3. This is the consequence of the fact that in all functionals (i) different single-particle subshells are well separated in energy below $N=126$ (see Fig. 1) and (ii) the sequence

\footnotetext{
7 It is only in the PC-PK1 functional that the $2 g_{9 / 2}$ subshell is lower in energy than the $1 i_{11 / 2}$ one for $N=126$ [see Fig. 1(b)]. However, already at $N=128$ the $1 i_{11 / 2}$ subshell dives below $2 g_{9 / 2}$ [see Fig. 5(b)].
}

of the single-particle subshells occupied with increasing neutron number is the same (see Table III). In order to see whether a similar situation persists for higher neutron numbers, we performed calculations with and without pairing for all even-even $\mathrm{Pb}$ isotopes located between the two-proton and the two-neutron drip lines (see Fig. 6). Note that we restrict the calculations to spherical shapes to see the sources (not affected by the shape changes) of major differences between the functionals. This is a somewhat hypothetical scenario since the calculations with deformation included indicate the presence of deformation in the ground states of the $\mathrm{Pb}$ isotopes located in the middle of the region between the magic neutron closures (see Fig. 19 of Ref. [4]). The neutron single-particle rms radii of the single-particle orbitals are shown in Table II. One can see very large neutron rms radii of the $3 d_{5 / 2}, 2 g_{7 / 2}, 3 d_{3 / 2}$ and, especially, of the $4 s_{1 / 2}$ subshells. However, as discussed in the introduction and in Ref. [6] the real impact of the occupation of these orbitals on the charge radii will be defined by the pull they exert on proton densities.

TABLE II. Neutron single-particle rms radii $r_{n e u}^{s p}=\sqrt{\left\langle r^{2}\right\rangle^{s p}}$ of the indicated single-particle orbitals obtained in ${ }^{208} \mathrm{~Pb}$ in calculations without pairing with the CEDF DD-ME2. The order [from top to bottom] of the orbitals is the same as in Fig. 1(b). The radii of the first two orbitals located above the $N=126$ spherical shell gap are shown in bold.

\begin{tabular}{|c|c|}
\hline s-p orbital & $r_{\text {neu }}^{s p}[\mathrm{fm}]$ \\
\hline 1 & 2 \\
\hline $3 d_{3 / 2}$ & 8.9411 \\
$2 g_{7 / 2}$ & 7.4880 \\
$4 s_{1 / 2}$ & 9.3128 \\
$3 d_{5 / 2}$ & 8.2905 \\
$2 g_{9 / 2}$ & $\mathbf{7 . 0 2 2 7}$ \\
$1 i_{11 / 2}$ & $\mathbf{6 . 4 1 3 1}$ \\
$3 p_{1 / 2}$ & 6.4775 \\
$3 p_{3 / 2}$ & 6.3856 \\
$2 f_{5 / 2}$ & 6.2215 \\
$1 i_{13 / 2}$ & 6.4108 \\
\hline
\end{tabular}

We start from the analysis of calculations performed without pairing (see upper curves in Fig. 6). The significant (comparable to that seen at $N=126$ ) changes of the slope of the $\delta\left\langle r^{2}\right\rangle^{N, 126}$ curves are observed at $N=138$ : at this neutron number the $1 i_{11 / 2}$ subshell is completely filled and the $2 g_{9 / 2}$ subshell is filled at higher neutron number (up to $N=148$ ). The slopes of the $\delta\left\langle r^{2}\right\rangle^{N, 126}$ curves for different CEDFs as a function of the neutron number are similar in the neutron range $N=126-148$, and these curves do not cross. However, the situation starts to change above $N=148$ because of the different sequences of the occupation of the single-particle subshells (see Table III) caused by the fact that five different subshells, clustered into an energy window which is slightly larger than $1 \mathrm{MeV}$ (see top of Fig. 1), have somewhat different relative energies for the different functionals. The NL3* and PC-PK1 functionals have the same 


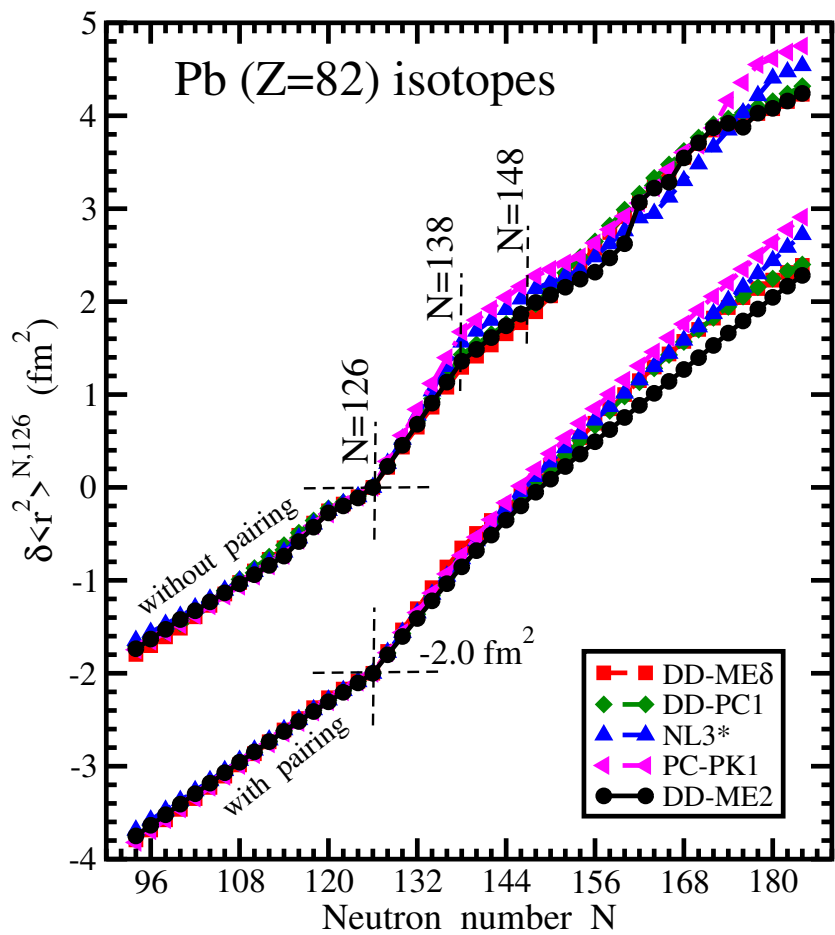

FIG. 6. The same as Figs. 2 and 3, but for all even-even $\mathrm{Pb}$ isotopes located between the two-proton and two-neutron drip lines. Note that the results of the calculations with pairing are shifted down by $-2.0 \mathrm{fm}^{2}$ in order to compare the results of the calculations with and without pairing on the same panel.

sequences of filling of spherical subshells with increasing neutron number (see column 1 in Table III). As a consequence, the $\delta\left\langle r^{2}\right\rangle^{N, 126}$ curves for these two functionals have comparable evolutions as a function of neutron number with minor changes of the slope at neutron numbers at which the transition from filling of one subshell to another one takes place. A similar situation also exists in the pair of functionals DD-PC1 and DD-ME $\delta$ (see Fig. 6) for which the sequences of the occupation of the single-particle subshells with increasing neutron number are the same (see column 2 in Table III). Note that the $\delta\left\langle r^{2}\right\rangle^{N, 126}$ values calculated with these two functionals are extremely close to each other.

The situation is more complex in the case of the DDME2 functional. Calculations with this functional reveal a complicated interplay of the occupation of different spherical subshells with increasing neutron number. The neutron $3 d_{5 / 2}$ and the $4 s_{1 / 2}$ subshells are gradually occupied for $N=149-156$ (see Table III). However, in the neutron number range $N=157-172$ a complicated interplay of the occupation of the $2 g_{7 / 2}$ and $1 j_{15 / 2}$ subshells and deoccupation of the $3 d_{5 / 2}$ and $4 s_{1 / 2}$ subshells with increasing neutron number take place [this region is labeled as "comp." in Table III]. It leads to substantial irregularities in the $\delta\left\langle r^{2}\right\rangle^{N, 126}$ curve at these particle numbers (see Fig. 6). At $N=172$ the $2 g_{7 / 2}$ and $1 j_{15 / 2}$ subshells are fully occupied, and the repeti-
TABLE III. The sequence of the spherical subshells occupied in the $\mathrm{Pb}$ isotopes with increasing neutron number in the calculations without pairing. Note that these sequences are the same for the pairs of the NL3* and PC-PK1 as well as DD$\mathrm{PC} 1$ and DD-ME $\delta$ functionals. The numbers in the brackets $\left[N_{1}-N_{2}\right]$ provide the range of neutron numbers between $N_{1}$ and $N_{2}$ for which the occupation of a given subshell takes place. The sequences of the subshells and neutron number ranges are shown in the columns 2 and 3 only starting from the point at which the difference with the column 1 emerges.

\begin{tabular}{|cc|cc|cc|}
\hline \hline \multicolumn{2}{|c|}{ NL3*/PC-PK1 } & DD-PC1/DD-ME $\delta$ & \multicolumn{2}{c|}{ DD-ME2 } \\
\hline & 1 & 2 & & 3 \\
\hline $1 i_{13 / 2}$ & {$[101-114]$} & & & & \\
$2 f_{5 / 2}$ & {$[115-120]$} & & & & \\
$3 p_{3 / 2}$ & {$[121-124]$} & & & & \\
$3 p_{1 / 2}$ & {$[125-126]$} & & & & \\
$1 i_{11 / 2}$ & {$[127-138]$} & & & & \\
$2 g_{9 / 2}$ & {$[139-148]$} & & & \\
$3 d_{5 / 2}$ & {$[149-154]$} & $1 j_{15 / 2}$ & {$[149-164]$} & $3 d_{5 / 2}$ & {$[149-154]$} \\
$2 g_{7 / 2}$ & {$[155-162]$} & $2 g_{7 / 2}$ & {$[165-172]$} & $4 s_{1 / 2}$ & {$[155-156]$} \\
$4 s_{1 / 2}$ & {$[163-164]$} & $3 d_{5 / 2}$ & {$[173-178]$} & comp. & {$[157-172]$} \\
$1 j_{15 / 2}$ & {$[165-180]$} & $4 s_{1 / 2}$ & {$[179-180]$} & $3 d_{5 / 2}$ & {$[173-178]$} \\
$3 d_{3 / 2}$ & {$[181-184]$} & $3 d_{3 / 2}$ & {$[181-184]$} & $4 s_{1 / 2}$ & {$[179-180]$} \\
& & & & $3 d_{3 / 2}$ & {$[181-184]$} \\
\hline
\end{tabular}

tive occupation of the $3 d_{5 / 2}$ and $4 s_{1 / 2}$ subshells as well as the occupation of the $3 d_{3 / 2}$ subshell takes place at higher neutron numbers.

The inclusion of pairing leads to a redistribution of the occupation of the single-particle states located in the vicinity of the neutron Fermi level and basically removes all the changes in the slopes of the $\delta\left\langle r^{2}\right\rangle^{N, 126}$ curves seen in the calculations without pairing for $N>126$ (compare the results of the calculations with and without pairing shown in Fig. 6). However, important differences between the functionals still exist. This is best illustrated by comparing the results obtained with the $\mathrm{DD}^{*}$ functionals. For these functionals $\delta\left\langle r^{2}\right\rangle^{184,126} \approx 4.3 \mathrm{fm}^{2}$ both in the calculations with and without pairing (see Fig. 6). At $N=184$, neutron pairing collapses (see, for example, Fig. 2 in Ref. [62]) but proton pairing is present due to the reduced size of the $Z=82$ shell closure as compared with the one for the $\mathrm{N}=126$ isotope. It is weakest for the DD-ME2 functional and strongest for the DD$\mathrm{ME} \delta$ one. This feature explains the slightly larger spread $\Delta\left(\delta\left\langle r^{2}\right\rangle^{184,126}\right)=0.117 \mathrm{fm}^{2}$ of the $\delta\left\langle r^{2}\right\rangle^{184,126}$ values obtained with the $\mathrm{DD}^{*}$ functionals in the calculations with pairing as compared without pairing.

Thus, the results of the $\mathrm{DD}^{*}$ functionals are the same at $N=126$ (by normalization) and almost the same at $N=184$. However, the difference between the DD-ME2 and DD-PC1/DD-ME $\delta$ functionals ${ }^{8}$ is increasing on moving away from the shell closures, and it is maximized at

\footnotetext{
${ }^{8}$ Note that the $\delta\left\langle r^{2}\right\rangle^{N, 126}$ curves obtained with the CEDFs DD$\mathrm{PC} 1$ and DD-ME $\delta$ are almost the same for all neutron numbers.
} 
$N=170$ where it reaches $0.302 \mathrm{fm}^{2}$ (see bottom curves of Fig. 6). This is due to different sequences of the filling of spherical subshells in these two groups of the functionals (see Table III).

Thus, the relative energies of the single-particle states and the patterns of their occupation with increasing neutron number are still important even in the calculations with pairing. They can lead to different predictions in different functionals and to discrepancies with experiment. It is important to remember that the group of the singleparticle subshells discussed above is located in a very narrow energy range $(\approx 1.0 \mathrm{MeV}$, see Fig. 1$)$. Thus, the correct description of the sequence of the occupation of the single-particle states with increasing neutron number requires an enormous accuracy (within approximately $200 \mathrm{keV}$ ) for the description of the energies of the single-particle states. Such an accuracy is unachievable in the present generation of energy density functionals (both covariant and non-relativistic ones). This is because the structure of the experimental ground states in odd- $A$ nuclei is reproduced globally only in approximately $40 \%$ of the nuclei in the DFTs, and there are substantial differences between experimental and calculated single-particle spectra [63-65]. The inclusion of particlevibrational coupling increases the accuracy of the description of the single-particle configurations in odd- $A$ nuclei but such studies are, so far, limited to spherical nuclei (see Refs. [66-68]).

The results of the calculations with PC-PK1 and NL3* functionals show the same trends in the $\delta\left\langle r^{2}\right\rangle^{N, 126}$ curves (see Fig. 6) reflecting the same sequence of the occupations of the spherical subshells (see Table III). At $N=184$, the $\delta\left\langle r^{2}\right\rangle^{184,126}$ values obtained with these two functionals are higher than those obtained with the DD* ones. This is most likely the consequence of the different isovector properties of the compared functionals (see Ref. [36]).

\section{CHARGE RADII IN ISOTOPIC CHAINS OF THE PB REGION}

The absolute values of experimental and calculated charge radii of the $\mathrm{Pt}, \mathrm{Hg}, \mathrm{Pb}, \mathrm{Po}$, and $\mathrm{Rn}$ isotopes are compared in Fig. 7, while a similar comparison for differential charge radii is presented in Fig. 12 below. Note that in these and in other figures, which cover the nuclei over the specific region, we consider only experimental even- $Z$ and calculated even-even nuclei. This is done in order to focus on general global features and avoid the discussion of odd- $A$ nuclei and related OES in charge radii which will be separately considered in Sec. IX below.

This is the consequence of (i) the same sequences of the filling of spherical subshells with increasing neutron number (see Table III) and (ii) similar isovector properties of these two functionals (see Table III in Ref. [36]).
The calculated quadrupole deformations of the lowest in energy solutions are presented in Fig. 8.

The relative charge radii of the $\mathrm{Pt}, \mathrm{Hg}$ and $\mathrm{Pb}$ isotopes are reasonably well reproduced in the region of neutron numbers around $N \approx 120$ (see Fig. 7 ). The increase of proton number above $Z=82$ leads to a gradual increase of charge radii in model calculations with all employed CEDFs (see Figs. 7(b), (c), (d), (e) and (f)). In contrast, there is a substantial gap in experimental charge radii of the $Z=82$ and $Z=84$ isotopes (see Fig. 7(a)) which is larger than that predicted in the calculations. The increase of charge radii in going on from the Po to $\mathrm{Rn}$ isotopes is somewhat smaller in experiment as compared with theoretical results. Note that at this point it is not clear whether these differences are due to the deficiencies of the model predictions or experimental evaluations of absolute values of charge radii (see discussion in the Introduction).

The evolution of experimental charge radii in the $R n$ isotopes are rather well reproduced in model calculations (see Fig. 7). Note that only the isotopes in the vicinity of the neutron shell closure at $N=126$ are spherical in the lowest in energy solutions (see Fig. 8). Some moderate deformation $|\beta \approx 0.10|$ appears in the calculations for experimentally known nuclei at $N=116-120$, but their potential energy curves (PEC) are very soft (see Fig. 1 in the supplemental material) so they are expected to be transitional. The shift from spherical to transitional nuclei does not trigger visible changes in charge radii in experiment since beyond mean-field effects are expected to smooth out this transition.

The evolution of charge radii in the Po isotopes is reproduced rather well from the isotope with the highest neutron number accessible in experiment down to $N=120$ in DD-ME $\delta, N=118$ in DD-ME2, NL3* and DD-PC1 and $N=116$ in PCPK1 CEDFs (see Fig. 7). At lower neutron numbers, the experimental charge radii gradually bend up so that at $N=108$ experimental $r_{c h}$ exceed the value defined from the trend of charge radii defined at $N=118-126$ by $\approx 0.04 \mathrm{fm}$. However this process is more abrupt in the calculations, since the calculated radii for $N=108-116$ exceed the above mentioned trend by $\approx 0.04 \mathrm{fm}$. In the calculations, this abrupt shift in calculated charge radii at $N \approx 118$ is caused by the transition from spherical to oblate shapes with $\beta_{2} \approx-0.18$ (see Fig. 8). These facts suggest that the $r_{c h}$ values of the $N=108$ and $N \approx 118$ [depends on functional] isotopes are rather well reproduced in the calculations, but the mean-field calculations fail to reproduce the gradual transition in $r_{c h}$ seen between $N=106$ and $N \approx 118$ isotopes. This gradual transition is most likely due to beyond mean-field effects since the nuclei in this neutron number range have soft PECs (see Fig. 2 in the supplemental material). In addition, the triaxiality could play a role in this gradual transition.

The charge radii of the $\mathrm{Pb}$ isotopes in the $N=$ $116-132$ range are well described in the model calculations of Sec. IV. Here we focus on more neutron-poor 


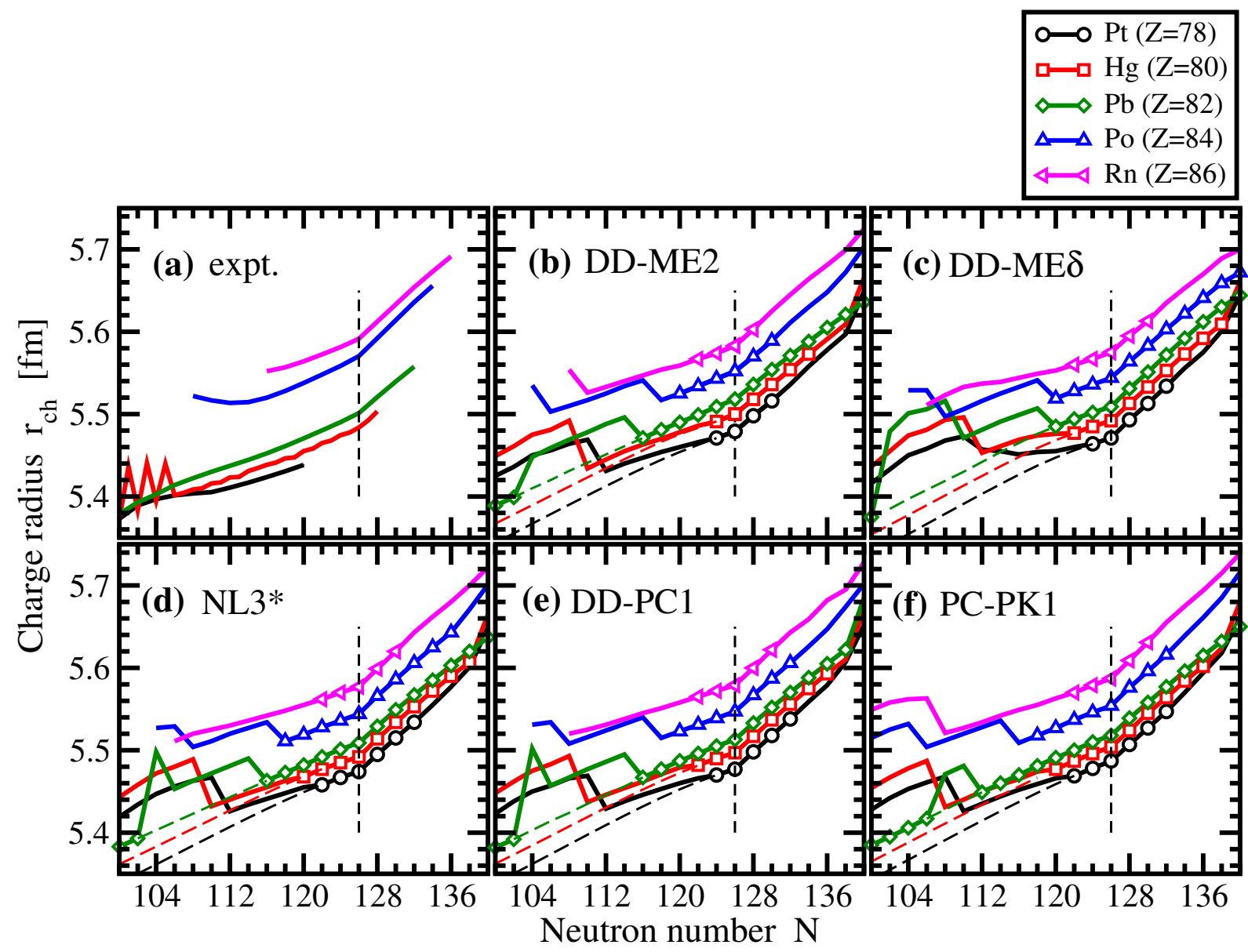

FIG. 7. The charge radii $r_{c h}$ of the $\mathrm{Pt}(Z=78), \mathrm{Hg}(Z=80), \mathrm{Pb}(Z=82), \mathrm{Po}(Z=84)$ and $\mathrm{Rn}(Z=86)$ isotopes as a function of neutron number. In panel (a), experimental data are shown only for even-even nuclei. The only exception is the Hg isotopes for which experimental data for odd- $N$ isotopes is included in order to illustrate a typical magnitude of OES in charge radii induced by shape coexistence (the $N=101-106$ region, see Sec. IX A for a detailed discussion) or by other effects when neighboring even and odd- $N$ isotopes have comparable shapes (the rest of the $\mathrm{Hg}$ curve). In panels (b)-(f), thick solid lines show the $r_{c h}$ values obtained in the calculations for the lowest in energy solutions in each isotopes. Open symbols show the isotopes for which these solutions are either spherical or quasi-spherical $\left(\left|\beta_{2}\right| \leq 0.05\right)$. This "line-symbol" convention is used in all figures below. Thin dashed lines show the charge radii of spherical solutions in neutron-poor $\mathrm{Pt}, \mathrm{Hg}$ and $\mathrm{Pb}$ isotopes. Vertical black dashed line indicates $N=126$.

$\mathrm{Pb}$ isotopes in the range of $N=100-116$. Experimental charge radii in this neutron range continue the trend seen at $N=116-126$ [see Fig. $7($ a)]. This suggests that the shapes of the nuclei in the measured states are either spherical or near-spherical. Indeed, if we consider spherical solutions in these nuclei (see green dashed lines in panels (b)-(f) of Fig. 7), then the experimental data are rather well reproduced. However, the calculations predict either oblate or prolate shapes for the ground states of the $N=104-114$ isotopes in CEDFs DD-ME2, NL3* and DD-PC1, of the $N=102-118$ isotopes in DD-ME $\delta$ and of the $N=108-110$ isotopes in PC-PK1 (see Fig. 8). Despite that, spherical minima, located either close in energy to the ground states or at some excitation energy, exist in all isotopes in all functionals with the exception of DD-ME $\delta$ (see Fig. 9). Note that PECs of these nuclei are rather soft in quadrupole deformation (see Fig. 9
). Thus, the correlations beyond mean-field can play an important role in these nuclei.

According to the droplet model (DM) [69] the following relation

$$
\left\langle r^{2}\right\rangle_{D M}=\left\langle r^{2}\right\rangle_{D M}^{\text {spher }}\left(1+\frac{5}{4 \pi} \beta_{2}^{2}\right)
$$

exists between the predictions of charge radii $\left\langle r^{2}\right\rangle_{D M}$ and $\left\langle r^{2}\right\rangle_{D M}^{\text {spher }}$ at quadrupole deformation $\beta_{2}$ and at spherical shape, respectively. This relation is frequently used in the experimental analysis of the data for the extraction of quadrupole deformations. This equation tells us that the charge radii form a parabolic function of $\beta_{2}$ with the minimum at spherical shape; this function is symmetric with respect to a sign change of the deformation. Realistic calculations presented in Fig. 10 confirm this parabolic-like dependence of charge radii on the quadrupole deforma- 


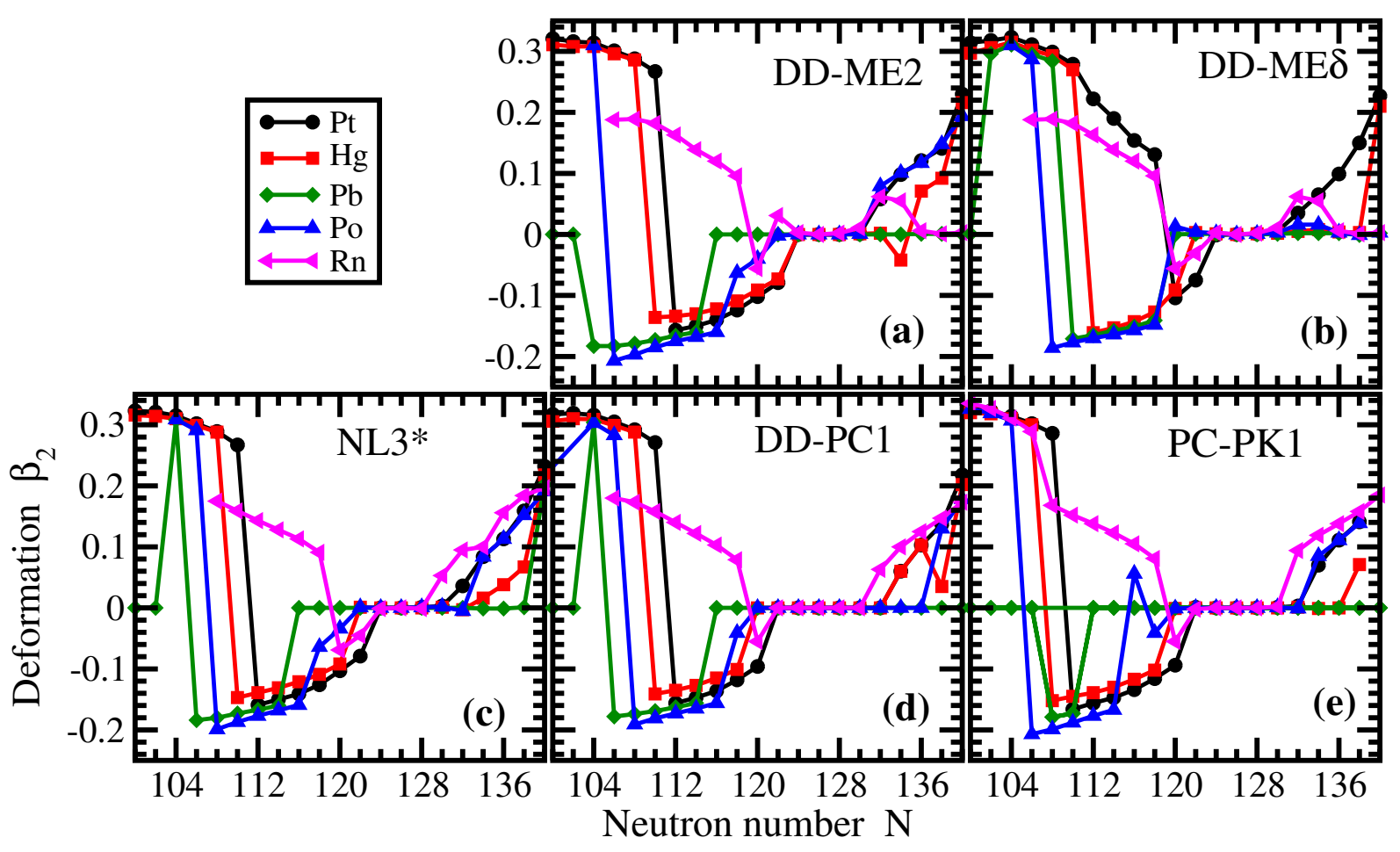

FIG. 8. Quadrupole deformations $\beta_{2}$ of the lowest in energy solutions of the $\mathrm{Pt}(Z=78), \mathrm{Hg}(Z=80), \mathrm{Pb}(Z=82)$, $\mathrm{Po}$ $(Z=84)$ and $\operatorname{Rn}(Z=86)$ isotopes obtained in the calculations with the indicated functionals.

tion. However, it is somewhat asymmetric with respect of the change of the sign of the deformation ${ }^{9}$. This difference between Eq. (9) and the results of Fig. 10 are most likely due to neglecting higher-order deformations (such as $\beta_{4}$ etc.) in Eq. (9). Note that the functional dependence of the charge radii on deformation $\beta_{2}$ almost does not depend on the functional (see Fig. 9). This fact is quite useful in the selection of the most probable scenario when comparing the experimental situation in the $\mathrm{Pb}$ region with the results of the calculations that provide several local closely lying minima.

Detailed investigations within the CDFT framework of charge radii in $\mathrm{Hg}$ isotopes with neutron numbers $N=$ $121-128$ are presented in Refs. [17, 71]. Good agreement between theory and experiment is obtained. The charge radii are also well described for neutron numbers $N=$ 110 - 120 in calculations with DD-ME2, NL3* and DDPC1, for $N=112-120$ with DD-ME $\delta$, and for $N=$ $108-120$ in PC-PK1 (see Fig. 7). However, they also suggest that these nuclei are oblate with $\beta_{2} \approx-0.15$ in their ground states (see Fig. 8 and Fig. 11) which leads to a slight increase of $r_{c h}$ as compared with the ones for the spherical solution (compare dashed red lines with solid

\footnotetext{
9 Note that Ref. [70] provides even higher order expansion of charge radii in terms of multipole deformations within the droplet model. However, the asymmetry of $\left\langle r^{2}\right\rangle_{D M}$ as a function of $\beta_{2}$ is opposite to that seen in the RHB calculations in Fig. 10 because of cubic term in $\beta_{2}$.
}

red lines with red squares in panels (b)-(f) of Fig. 7). A significant odd-even staggering in the $\mathrm{Hg}$ charge radii exists for $N=100-106$ [see Fig. 7(a)] the origin of which is discussed in Sec. IX A.

Except for calculations with the DD-ME $\delta$ functional, the evolution of experimental data in the Pt isotopes is rather well reproduced for the $N=112-120$ nuclei. The results of the calculations with DD-ME2, NL3*, DD-PC1 and PC-PK1 suggest that the ground states of these nuclei have weakly deformed oblate shapes with $\beta_{2} \approx-0.13$ (see Fig. 3 in the supplemental material). However, the competing prolate minimum exists in all these isotopes. With decreasing neutron number, this prolate minimum with $\beta_{2} \approx 0.3$ becomes the lowest in energy at $N=110$ (see Fig. 8 and Fig. 3 in the supplemental material). This leads to a sharp increase in charge radii which overshoots the experimental data by roughly $0.04 \mathrm{fm}$ (see Fig. 7). However, if we would associate excited oblate states with deformation $\beta_{2} \approx-0.2$ in the $N=100-110$ nuclei with the observed ground states, then the experimental data on charge radii would be much better described since they are characterized by lower charge radii (see discussion of Fig. 10 above). The change of the slope of experimental charge radii at $N \approx 106$ is possibly an indicator of such a transition from oblate shapes with nearly constant $\beta_{2} \approx-0.2$ for $N=100-106$ to oblate shapes where the deformation decreases in absolute value with increasing neutron number above $N=106$ (see Fig. 8 and Fig. 3 in the supplemental material). 


\section{$\mathrm{Pb}(\mathrm{Z}=82)$}

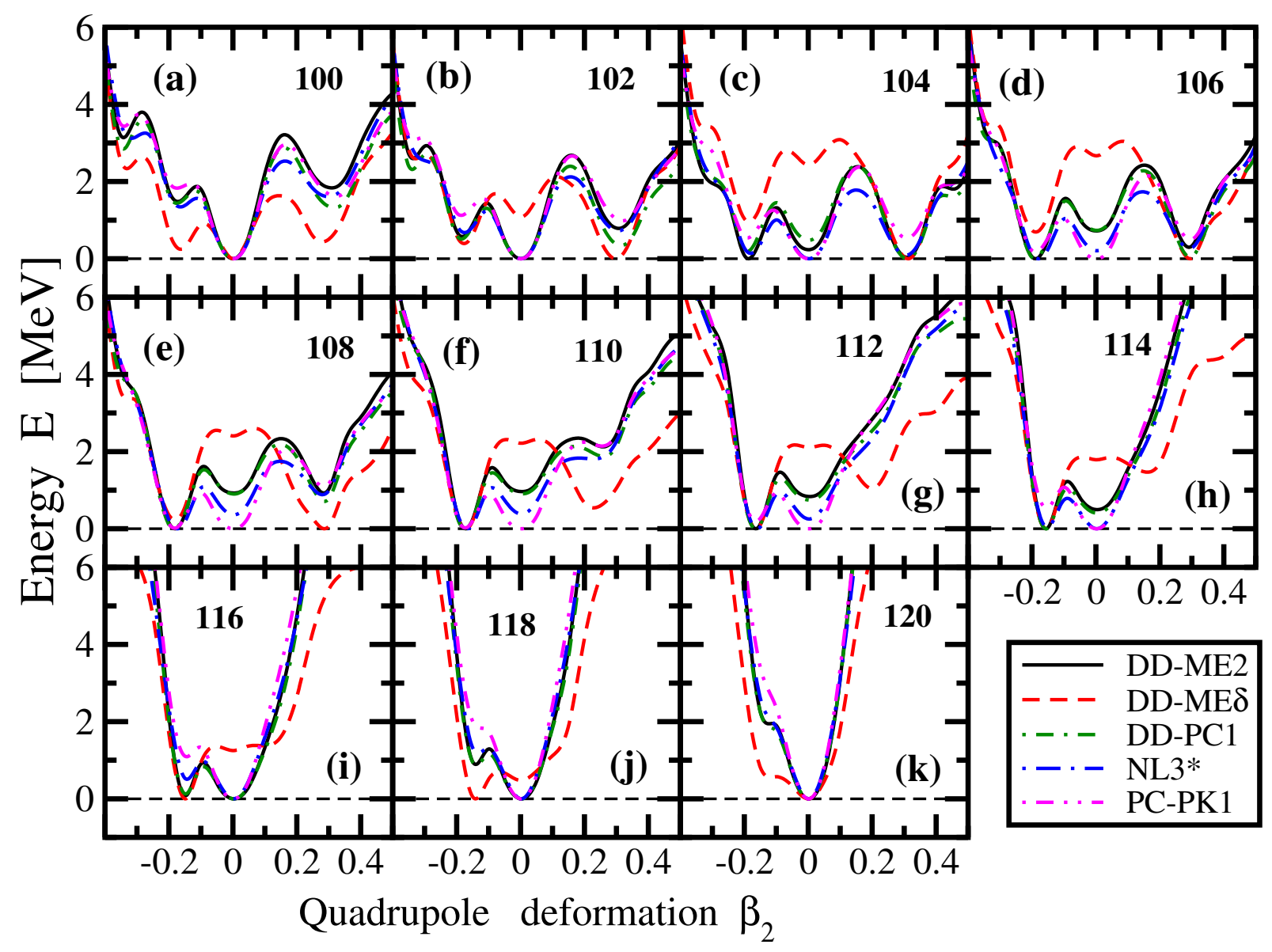

FIG. 9. Potential energy curves (PECs) of the Pb isotopes with neutron numbers $N=100-120$ as a function of quadrupole deformation $\beta_{2}$ obtained in the RHB calculations with the indicated functionals. Note that their energies are normalized to zero at the global minimum.

Fig. 12 shows the evolution of the $\delta\left\langle r^{2}\right\rangle^{N, 126}$ values in the $\mathrm{Pt}, \mathrm{Hg}, \mathrm{Pb}, \mathrm{Po}$, and $\mathrm{Rn}$ isotopes. These curves are similar [clustered] for different isotopic chains for $N=112-136$, but their slopes change at $N=126$. Below $N=112$ this feature is disturbed in the Po isotopes due to the gradual transition to prolate shapes, but still, it is present for the isotopic chains of $\mathrm{Pb}, \mathrm{Hg}$ (excluding odd nuclei), and $\mathrm{Pt}$. This clustering of the $\delta\left\langle r^{2}\right\rangle^{N, 126}$ values for different isotopic chains is well reproduced in all functionals above $N=126$. The situation is somewhat different below $N=126$. The DD-ME2, NL3*, DD$\mathrm{PC} 1$ and PC-PK1 functionals reproduce this clustering of the $\delta\left\langle r^{2}\right\rangle^{N, 126}$ values down to $N \approx 116$ and for the $\mathrm{Pt}$, $\mathrm{Hg}$, and $\mathrm{Rn}$ isotopic chains even to lower neutron numbers. However, as discussed above in the low- $N$ region the calculations do not reproduce correctly the lowest in energy minimum leading to the discrepancies between theory and experiment. Note that among the functionals under consideration DD-ME $\delta$ provides the worst description of experimental data in the nuclei with $N<126$.

\section{CHARGE RADII IN ISOTOPIC CHAINS OF THE SN/GD REGION}

The calculated and experimental differential charge radii for the $\mathrm{Sn}$ isotopes are compared in Fig. 13. The experimental $\delta\left\langle r^{2}\right\rangle^{N, 74}$ curve is well described in the $N=58-74$ range in model calculations with the best reproduction provided by the NL3*, DD-ME $\delta$ and DDPC1 functionals. However, the slope of the experimental $\delta\left\langle r^{2}\right\rangle^{N, 74}$ curve is somewhat overestimated in the $N=74-82$ range by all functionals. The experimental data shows a kink at $N=82$, the magnitude of which is underestimated by the NL3*, PC-PK1, DD-ME2 and DD-PC1 CEDFs. Only the DD-ME $\delta$ functional reproduces it reasonably well with the slope of the experimental $\delta\left\langle r^{2}\right\rangle^{N, 82}$ curve for $N>82$ described almost perfectly (see inset in Fig. 13).

The origin of this feature can be traced back to the occupation pattern of the neutron $1 h_{9 / 2}$ and $2 f_{7 / 2}$ subshells located above the $N=82$ shell closure (see Fig. 


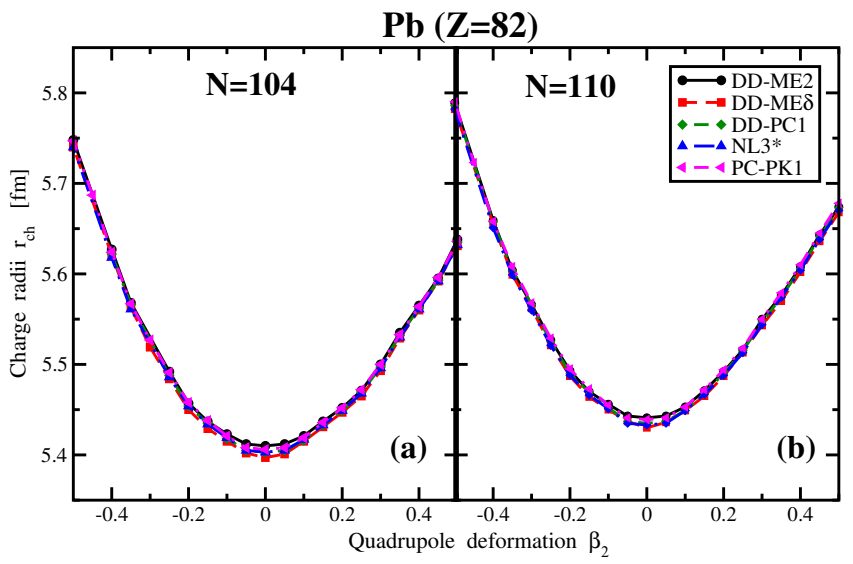

FIG. 10. The variations of charge radii $r_{c h}$ in ${ }^{186} \mathrm{~Pb}$ and ${ }^{192} \mathrm{~Pb}$ nuclei as a function of the quadrupole deformation obtained in RHB calculations with the indicated functionals.

14(a)). The calculations without pairing show that the occupation of the $2 f_{7 / 2}$ orbital, which is the lowest in energy subshell above $N=82$ (see Fig. 14(a)), does not create a kink at $N=82$ (see inset in Fig. 13). A similar situation exists for the occupations of other orbitals such as the $n=2$ orbital $2 f_{5 / 2}$ and the $n=3$ orbitals $3 p_{3 / 2}$, and the $3 p_{1 / 2}$ orbitals located above $N=82$. It is only the occupation of the $n=11 h_{9 / 2}$ orbital which drives the $N=84$ isotope to a visibly larger charge radius and creates a kink at $N=82$ (see inset in Fig. 13). This situation is very similar to the one seen in the $\mathrm{Pb}$ isotopes in which only the occupation of the $n=11 i_{11 / 2}$ subshell above $N=126$ creates a kink in charge radii at this particle number (see Sec. IV).

Pairing leads to a redistribution of the occupation of different single-particle orbitals in the $N=84$ isotope of $\mathrm{Sn}$ (see Fig. 15). However, it is only for the DD-ME $\delta$ functional that the $1 h_{9 / 2}$ orbital is strongly occupied in the RHB calculations [stronger than the $2 f_{7 / 2}$ one] because of the closeness of the $2 f_{7 / 2}$ and $1 h_{9 / 2}$ orbitals in energy (see Fig. 14(b)). And this balance of the occupation of these orbitals leads to an almost perfect reproduction of the slope of differential charge in the $N>82$ $\mathrm{Sn}$ isotopes (see inset in Fig. 13). In contrast, in other functionals the $1 h_{9 / 2}$ orbital is higher in energy by approximately $2 \mathrm{MeV}$ than the $2 f_{7 / 2}$ one (see Fig. $14(\mathrm{~b})$ ) and, as a result, its occupation probability is by a factor three or four [dependent on CEDF] smaller than that for $2 f_{7 / 2}$ (see Fig. 15). As a consequence, the slope of differential charge radii in the $N>82$ isotopes as well as the magnitude of the kink at $N=82$ is underestimated by these functionals (see inset in Fig. 13).

The experimental and calculated differential charge radii in the $\mathrm{Cd}, \mathrm{Sn}, \mathrm{Te}, \mathrm{Xe}, \mathrm{Ba}, \mathrm{Ce}, \mathrm{Nd}, \mathrm{Sm}$, and $\mathrm{Gd}$ isotopic chains are compared in Figs. 16 and 17. One can see that, on average, the experimental data are well reproduced.

For the nuclei with $N<82$, the experimental $\delta\left\langle r^{2}\right\rangle^{N, 82}$ curves diverge away from each other with de- creasing neutron number (see Fig. 17). The only exceptions from this trend are the $\mathrm{Cd}(Z=48)$ and $\mathrm{Sn}$ $(Z=50)$ isotopic chains, for which the experimental differential charge radii are almost the same. These features are best reproduced by the NL3* functional [compare panels (a) and (d) of Fig. 17]. The similarity of differential charge radii in the $\mathrm{Cd}$ and $\mathrm{Sn}$ isotopic chains is reproduced in all functionals. Note that the ground states of all $\mathrm{Sn}$ isotopes are predicted to be spherical (see Figs. 4 and 12 in the supplemental material). On the contrary, the $N=56-62 \mathrm{Cd}$ isotopes are predicted to be slightly prolate with $\beta_{2} \approx 0.15$ (see Fig. 4 in the supplemental material), but the PECs of many of them are soft in quadrupole deformation (see Fig. 13 in the supplemental material) so that the effects beyond mean-field could play a role in the definition of the exact ground state deformation. The relative properties of differential charge radii of the Te $(Z=52)$ and Sn isotopes are reproduced rather well in all functionals. Note that the calculations predict that many of the Te nuclei are soft in their ground states (see Fig. 11 in the supplemental material).

The evolution of differential charge radii in the $\mathrm{Xe}$ $(Z=54)$ isotopes and the kink at $N=82$ are reasonably well described by all functionals [see Fig. 16(d)]. However, some functionals slightly underestimate the experimental data in the $N<82$ nuclei. The relative properties of differential radii of the $\mathrm{Xe}$ and Te isotopic chains are somewhat better described by the DD-ME $\delta$ functional (compare panels (c) and (a) in Fig. 17). Note that in this functional the $N=64-68$ isotopes are predicted to be oblate with $\beta_{2} \approx-0.2$, while other isotopes are prolate with $\beta_{2} \approx 0.2$ (see Fig. $4(\mathrm{~b})$ in the supplemental material). In contrast, in other functionals the calculated ground state deformation is prolate at $N=64-68$, and it reaches a maximum value at $N \approx 66$ (see Figs. 4(a), (c), (d) and (e) in the supplemental material). As a result, the differential radii show a small peak at these neutron numbers (see Figs. 17(b), (d), (e) and (f)). However, the excited oblate minimum in those functionals is only slightly higher in energy than the prolate one (see Fig. 10 in the supplemental material). If one associates this oblate minimum with the ground state in the $N=64-68$ nuclei, then the experimental differential charge radii will be well reproduced by model calculations [see dashed green lines in Figs. 17(b), (d), (e) and (f)].

Experimental data on differential charge radii of the $\mathrm{Ba}(Z=56)$ isotopes extends down to $N=64$ with $\delta\left\langle r^{2}\right\rangle^{N, 82}$ values being nearly constant but slightly decreasing with decreasing neutron number [see Fig. 16(e)]. Similar trends are observed in the calculations with all CEDFs which reasonably well reproduce the evolution of experimental $\delta\left\langle r^{2}\right\rangle^{N, 82}$ curve as well as the kink at $N=82$. However, the calculated curves are disturbed by a small peak at $N=68$ in the calculations with DDME2, NL3*, DD-PC1, and PC-PK1 [see Figs. 17(b), (d), (e) and (f)] and a substantial downslope of the $\delta\left\langle r^{2}\right\rangle^{N, 82}$ curve with decreasing neutron number which starts at 
$\mathrm{Hg}[\mathrm{Z}=80]$

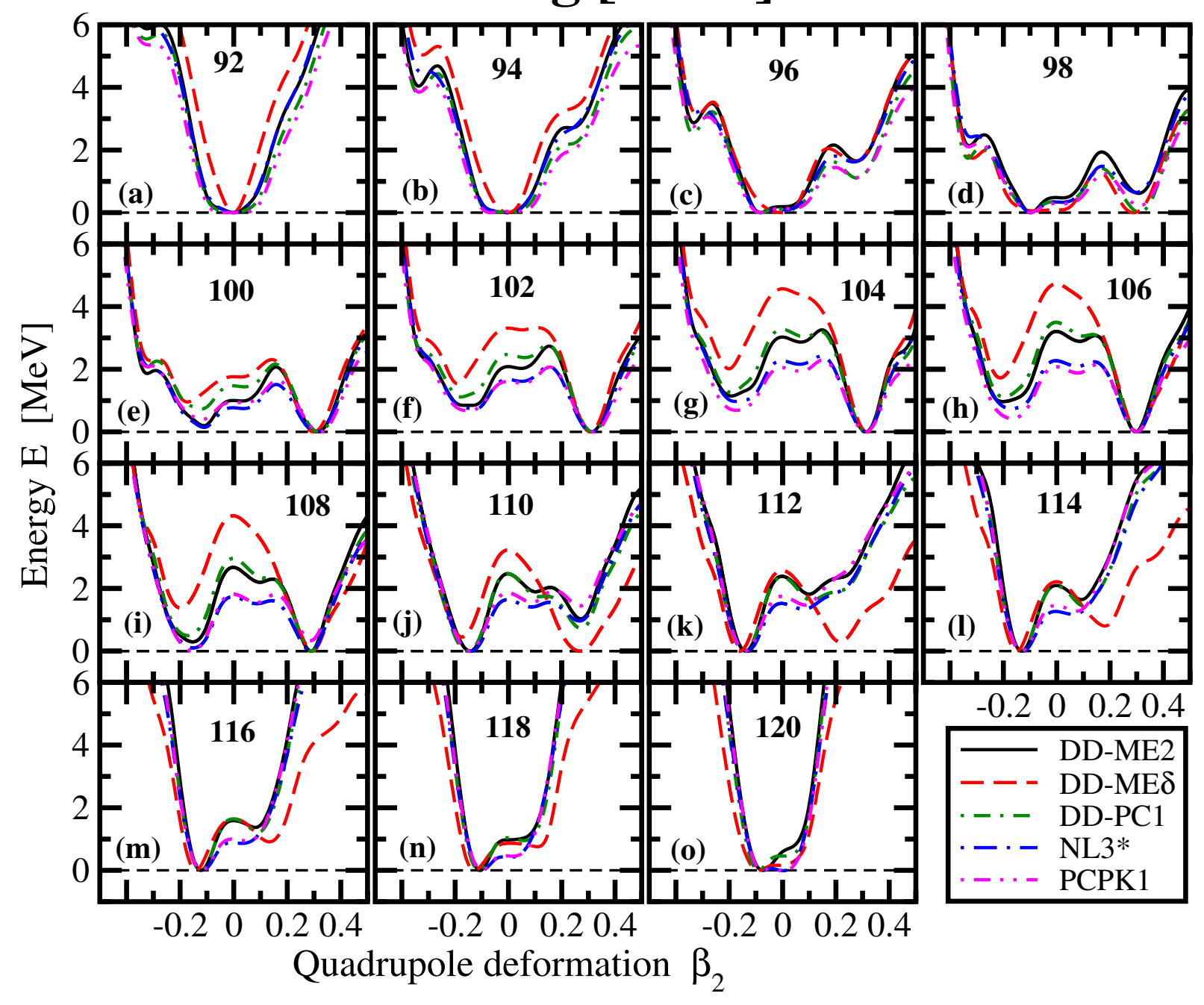

FIG. 11. The same as Fig. 9 but for the $\mathrm{Hg}$ isotopes with $N=100-120$.

$N=64$ in the calculations with DD-ME $\delta$ [see Figs. 16(e) and Fig. 17(c)]. This peak takes place at neutron numbers where the rate of the increase of prolate deformation with decreasing neutron number is enhanced (see Fig. 4 in the supplemental material).

In the $N<82$ nuclei, the experimental data for eveneven Ce $(Z=58)$ isotopes are available only for $N=78$ and 80 . It is reasonably well described in all model calculations [see Fig. 16(f)]. The experimental $\delta\left\langle r^{2}\right\rangle^{N, 82}$ values of the $\mathrm{Nd}(Z=60)$ isotopes are close to zero for $N=72-82$ and this feature is described by all functionals [see Fig. 16(g)]. The only exception is the $N=72$ isotope in the calculations with the CEDFs DD-ME2 and DD-PC1 for which a substantial increase of differential charge radii is predicted [see Figs. 16(g) and 17(b), (e)]. This is caused by the drift of the prolate minimum from $\beta_{2} \approx 0.2$ to $\beta_{2} \approx 0.4$ (see Fig. 7 in the supplemental material). The experimental $\delta\left\langle r^{2}\right\rangle^{N, 82}$ values of the $\mathrm{Sm}$ $(Z=62)$ isotopes are slightly higher than those of the $\mathrm{Nd}$ ones and are decreasing with increasing neutron number in the $N=76-80$ range [see 16(h)]. These two features are reasonably well described in the model calculations. The only exception is the DD-ME $\delta$ functional which does not predict this decreasing trend [see Figs. 17(c)].

Let us consider the evolution of differential charge radii in the $N>82$ nuclei. Note that experimental data for the $\mathrm{Cd}$ isotopes stops at $N=82$ and the one for the $\mathrm{Sn}$ and Te isotopic chains at $N=84$. The isotopic Ce and $\mathrm{Nd}$ chains extend up to $N=90$, those of $\mathrm{Xe}, \mathrm{Ba}$, and Sm up to $N=92$, and the Gd isotopic chain up to $N=96$. The substantial kink in charge radii at $N=$ 82 is present in all these isotopic chains; the $\delta\left\langle r^{2}\right\rangle^{84,82}$ values range from $0.226 \mathrm{fm}^{2}$ in $\mathrm{Sn}$ up to $0.297 \mathrm{fm}^{2}$ in Gd. These kinks are reasonably well described in the majority of the calculations (see Fig. 16). Note also that the calculations reasonably well reproduce the $N>82$ branches of differential charge radii.

The experimental differential charge radii of the in- 


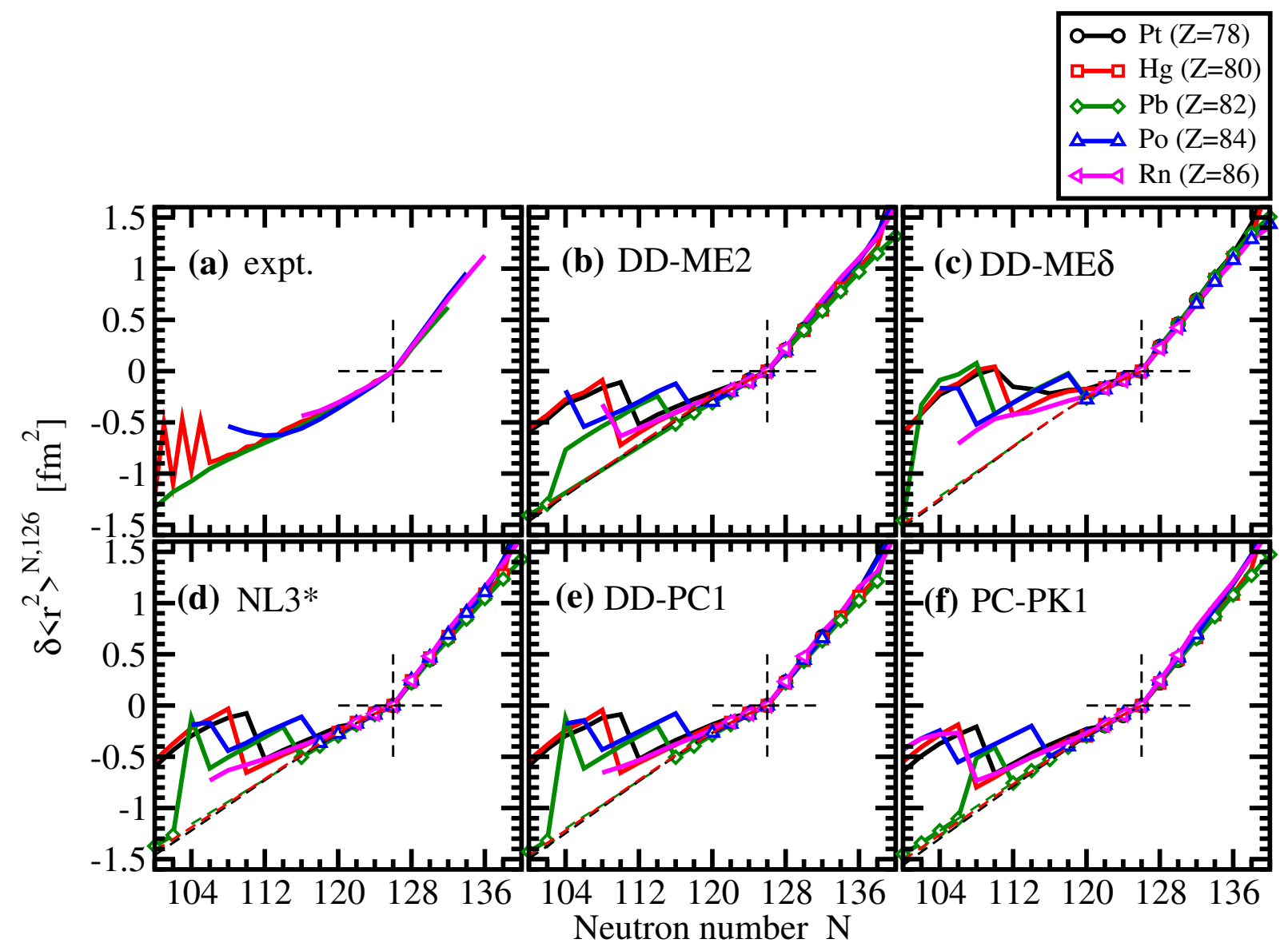

FIG. 12. The $\delta\left\langle r^{2}\right\rangle^{N, 126}$ values of the $\mathrm{Pt}(Z=78), \mathrm{Hg}(Z=80), \mathrm{Pb}(Z=82), \mathrm{Po}(Z=84)$ and Rn $(Z=86)$ isotopes as a function of neutron number. Thick lines (with open symbols for spherical of quasi-spherical solutions) show the $\delta\left\langle r^{2}\right\rangle^{N, 126}$ values obtained for the lowest in energy solutions in each isotopes. Thin dashed lines show the charge radii of spherical solutions in neutron-poor $\mathrm{Pt}, \mathrm{Hg}$ and $\mathrm{Pb}$ isotopes. Vertical black dashed line indicates $N=126$ and horizontal dashed line $\delta\left\langle r^{2}\right\rangle^{N, 126}=0$.

dicated isotopic chains cluster in the $N>82$ nuclei [see Fig. 17(a)] and this feature is reproduced well only in the calculations with DD-ME $\delta$ (compare panels (c) and (a) in Fig. 17). The spread of the $\delta\left\langle r^{2}\right\rangle^{90,82}$ values obtained in the calculations with DD-ME2, NL3*, DD-PC1, and PC-PK1 for the isotopic chains under study is larger by a factor of approximately two than that seen in the experiment (compare panels (b), (d), (e), and (f) with panel (a) in Fig. 17). Note that theoretical results for the Cd, Sn, and Te isotopic chains have to be ignored in such a comparison since experimental data in these chains extends only up to either $N=82$ or 84 .

The calculations indicate that only the $N=82$ and $84 \mathrm{Xe}, \mathrm{Ba}, \mathrm{Ce}, \mathrm{Nd}, \mathrm{Sm}$, and $\mathrm{Gd}$ isotopes are spherical in their ground states, while higher $N$ isotopes are prolate [with a pair of exceptions for DD-ME $\delta$ ] (see Fig. 4 in the supplemental material). This suggests that the clustering of differential charge radii of these isotopic chains has to be, in part, traced back to the similarity of calculated deformations. Indeed, in the neutron number range of $82-92$, the smallest spread of calculated deformations is seen in the calculations with DD-ME $\delta$ (see Fig. 4(b) in the supplemental material), and this functional provides the best description of the clustering (see Fig. 17). However, this is probably not the complete picture since the spread of calculated deformations for DD-PC1 is only slightly higher than in the calculations with DD-ME $\delta$ (compare panels (b) and (d) in Fig. 4 of the supplemental material), but it does not produce a good description of clustering. As a result, alternative sources of the clustering of differential charge radii for the nuclei above shell closures may be possible. One possibility is that it is related to the lowering of the energy of the $1 h_{9 / 2}$ neutron subshell to the vicinity of the $N=82$ shell closure, which is present only for the DD-ME $\delta$ functional (see Fig. 14).

It is interesting to compare the situation with the clustering of differential charge radii above the neutron shell closure in the $\mathrm{Pb}$ and $\mathrm{Sn}$ regions. This clustering in the $\mathrm{Pb}$ region for $N>126$ is defined by only three isotopic chains, namely, $\mathrm{Pb}, \mathrm{Po}$, and $\mathrm{Rn}$ (see Fig. 12). Moreover, the calculations suggest that the nuclei in the $N=126-130$ range are spherical, and that only a weak 


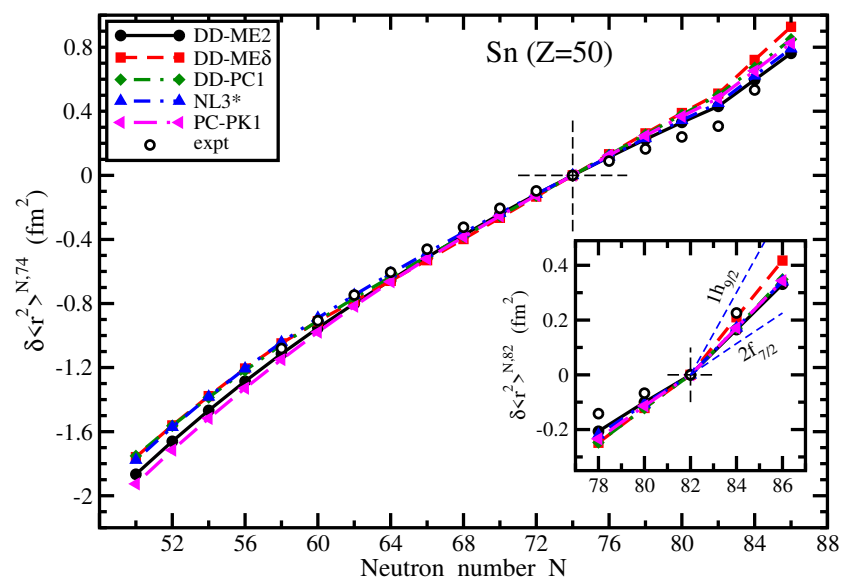

FIG. 13. The same as Fig. 3 but for the Sn isotopes. The experimental data are taken from Ref. [15]. The $N=74$ and $N=82$ nuclei are used as reference nuclei in the full figure and in the inset, respectively. The latter is done in order to show the kink at $N=82$ in more detail. The dashed lines in the inset show the results of the calculations without pairing and with the CEDF NL3* in which either only neutron $1 h_{9 / 2}$ or only neutron $2 f_{7 / 2}$ orbitals are occupied in the $N>82$ nuclei.

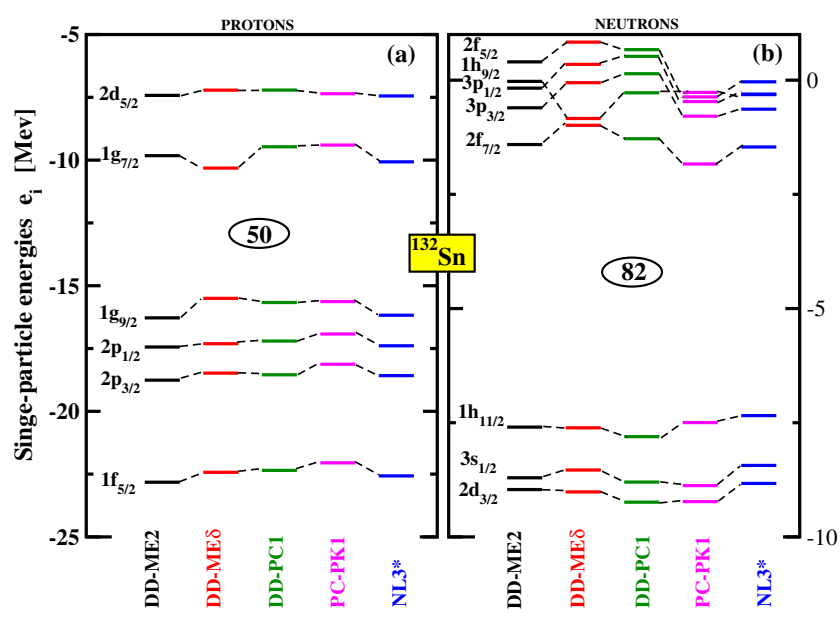

FIG. 14. The same as Fig.1 but for ${ }^{132} \mathrm{Sn}$.

deformation $\left(\left|\beta_{2} \approx 0.1\right|\right)$ develops for $N=132,134$, and 136 (see Fig. 8), the highest neutron numbers in the experimentally observed isotopic chains of $\mathrm{Pb}, \mathrm{Po}$, and $\mathrm{Rn}$, respectively. So, the latter nuclei remain quasi-spherical. All these factors explain why it is easier to reproduce the clustering of differential charge radii in the $\mathrm{Pb}$ region as compared with the $\mathrm{Sn} / \mathrm{Gd}$ one.

\section{THE CHARGE RADII IN THE ISOTOPIC CHAINS OF THE SR REGION}

The isotopic chains of $\mathrm{Kr}, \mathrm{Sr}$, and Mo allow us to test how the differential radii are modified when the $N=$ 50 shell closure is crossed. In Fig. 18 the experimental

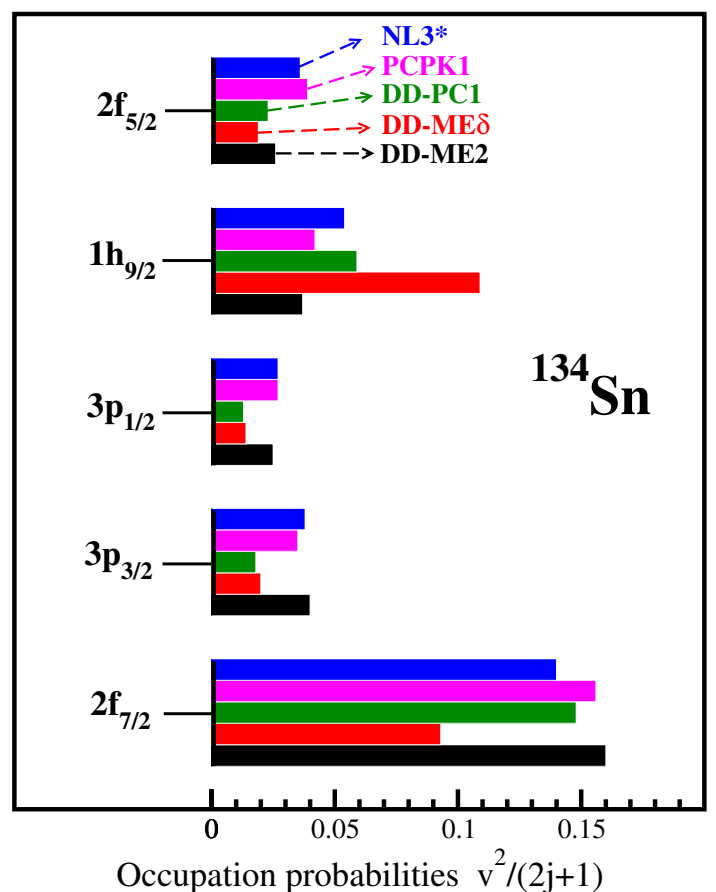

FIG. 15. The occupation probabilities $v^{2} /(2 j+1)$ of the neutron orbitals of ${ }^{134} \mathrm{Sn}$ located above the $N=82$ shell closure.

data for these chains are compared with the results of the calculations.

Let us first consider the differential radii for the $N>50$ isotopes. In the $\mathrm{Kr}$ isotopes, the calculations reproduce rather well the experimental data for $N=52$ and 54 , but start to overestimate it for $N=56,58$ and 60 with the biggest overestimate given by the $\mathrm{DD}^{*}$ functionals [see Fig. 18(a)]. All functionals rather well reproduce the experimental differential radii of the $\mathrm{Sr}$ isotopes for $N=$ 50-58 [see Fig. 18(b)]. Note that in both isotopic chains, the ground states have moderately oblate deformation with $\beta_{2} \approx-0.18$ above $N=52$ (see Figs. 14 and 16 in the supplemental material). Except for PC-PK1, the differential charge radii of the Mo isotopes are also rather well reproduced for $N=50-58$ [see Fig. 18(c)]. Note that the calculations show a competition of prolate and oblate shapes in this neutron number range (see Fig. 14 in the supplemental material).

The differential charge radii drastically increase for $N=60$ and 62 in the $\mathrm{Sr}$ isotopes and $N=60,62$, and 64 in the Mo isotopes [see Figs. 18(b) and (c)]. This increase is related to the transition of the ground states to a prolate minimum with $\beta_{2} \approx 0.4$. Such a minimum is excited by $100-200 \mathrm{keV}$ in the $\mathrm{Sr} N=60$ and 62 isotopes in the calculations with DD-ME2, DD-PC1, and NL3* (see Figs. 16 (n) and (o) in the supplemental material), and by approximately $1 \mathrm{MeV}$ as compared with the oblate ground state minimum in the $N=60,62$, and 64 Mo isotopes (see Figs. 15 (l), (m) and (n) in the supplemental material). Note that for some functionals and 


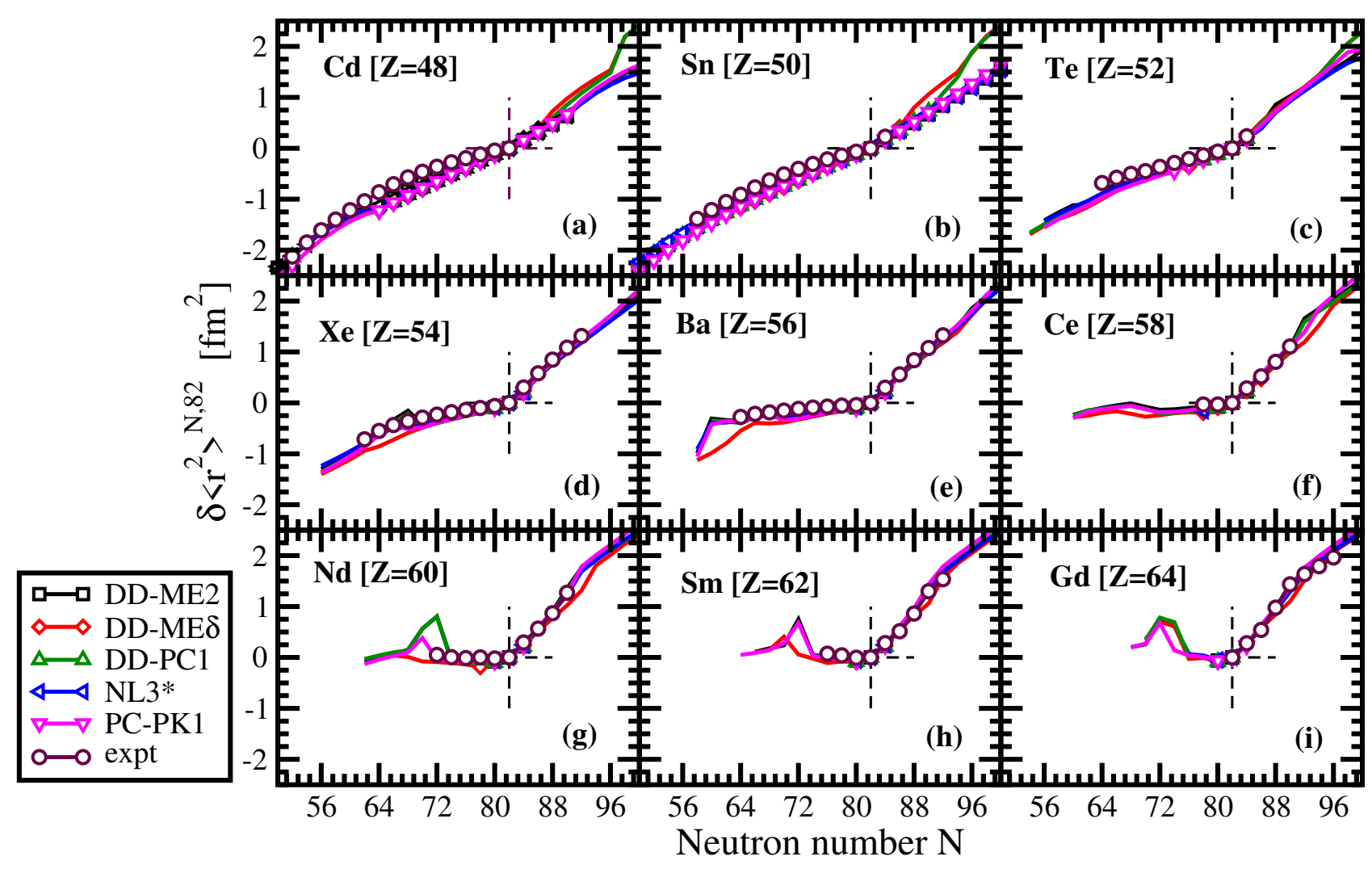

FIG. 16. The $\delta\left\langle r^{2}\right\rangle^{N, 82}$ values of the isotopic chains in the $\mathrm{Sn} / \mathrm{Gd}$ region. The experimental data are taken from Ref. [2].

some neutron numbers such a minimum is either nonexistent [appears as shoulder in the PEC] or separated by a very small barrier from the minima with lower deformation. Figs. 18(b) and (c) illustrates that experimental $\delta\left\langle r^{2}\right\rangle^{N, 50}$ values for these neutron numbers are rather well reproduced if the calculated charge radii in this minimum are used for comparison.

It is interesting to compare the performance of relativistic and non-relativistic functionals in the description of differential charge radii in the $N>50$ nuclei of the $\mathrm{Sr}$ region. The results of the calculations with nonrelativistic Skyrme functionals for the Sr isotopes are presented in Fig. 20. The best reproduction of experimental data in the $N=50-58$ range is provided by the SLy4 functional, but in general, the Skyrme functionals provide a less accurate description of the $\delta\left\langle r^{2}\right\rangle^{N, 50}$ values in this neutron number range as compared with the CEDFs. At higher neutron number, except for the SkP functional, the prolate minimum with $\beta_{2} \approx 0.37$ is the lowest in energy in the Skyrme DFT calculations, and this allows explaining the experimental data at $N=60$ and 62 .

Similar results have also been obtained in Ref. [32] in calculations with Gogny D1S for the Sr, Mo, and $\mathrm{Zr}$ isotopic chains. They underestimate experimental $\delta\left\langle r^{2}\right\rangle^{N, 50}$ values for $N=52-58$ but correctly predict the existence of a highly deformed prolate minimum above
$N=58$. This minimum is the lowest in energy in the $\mathrm{Sr}$ isotopes but an excited configuration in Mo. Moreover, similar to our results for these shapes, they overestimate $\delta\left\langle r^{2}\right\rangle^{N, 50}$ values for the Sr isotopes, but correctly reproduce them for Mo. Despite all these differences, both relativistic and non-relativistic functionals predict a similar trend of the evolution of charge radii with increasing neutron numbers above $N=50$ provided that the correct minimum is associated with the experimental data.

Both covariant and non-relativistic DFTs fail to reproduce the evolution of experimental $\delta\left\langle r^{2}\right\rangle^{N, 50}$ curve in the $N=40-50 \mathrm{Kr}$ and $\mathrm{Sr}$ isotopes (see Figs. 18 and 20 in the present paper and Fig. 7 in Ref. [72]). This is, because in the absolute majority of the cases, these models predict spherical ground states for these isotopes (see Figs. 14, 15, 16 and 17 in the supplemental material for CDFT results, Fig. 20 and Mass Explorer at FRIB [37] for Skyrme DFT results and Ref. [73] for Gogny DFT results). However, the PECs obtained in the CDFT calculations indicate the presence of a prolate minimum with $\beta_{2} \approx 0.5$ in the $N=36-42 \mathrm{Sr}$ isotopes (see Figs. 16 (b), (c), (d) and (e) in the supplemental material) which [with the exception of the calculations with a few functionals in the $N=38$ isotope] is the excited one. The calculated charge radii in this minimum somewhat overestimate experimental data [see the lines without symbols in Fig. 18(b)]. The existence of similar prolate minima is seen also in the Skyrme DFT calcula- 


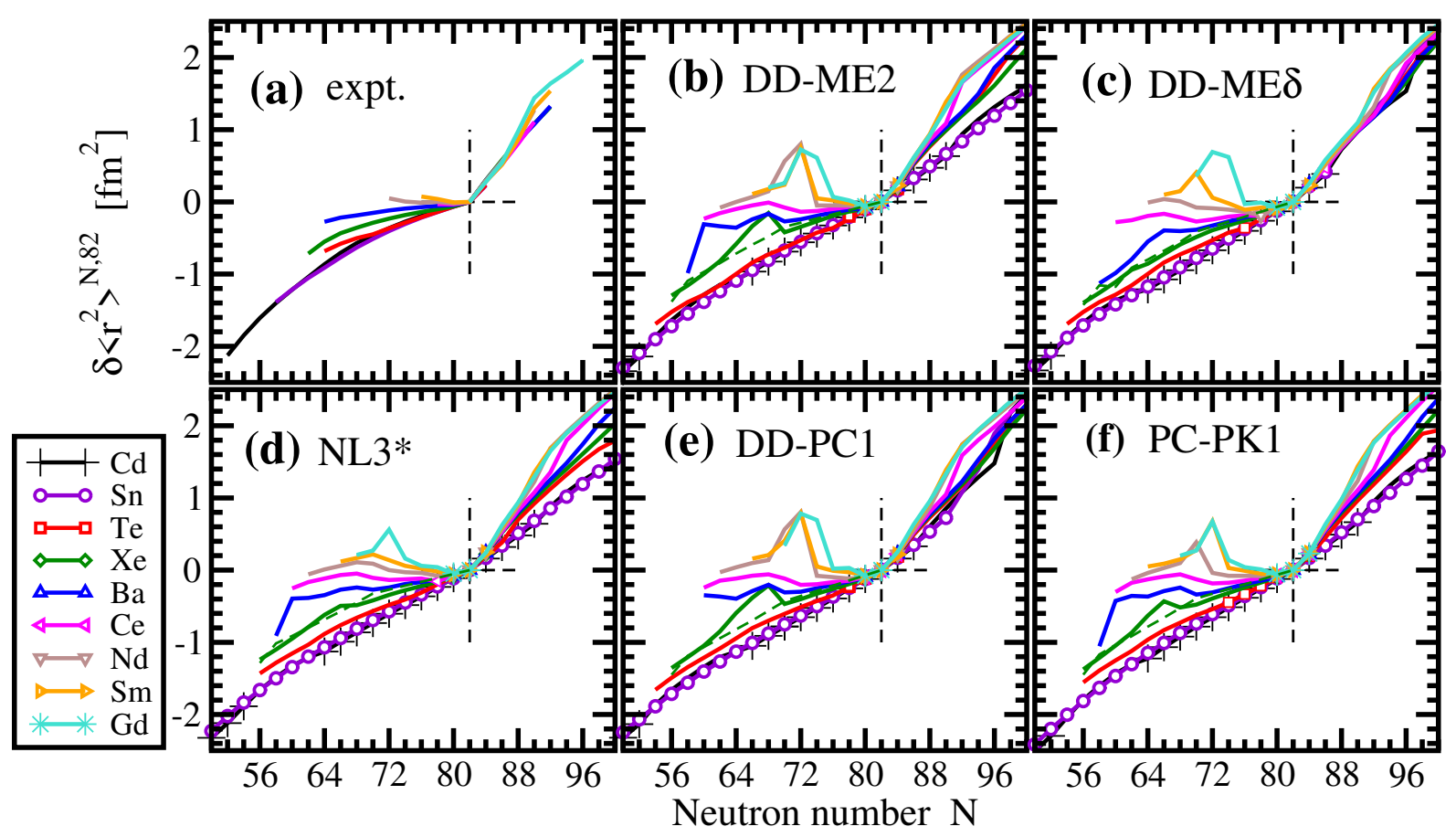

FIG. 17. The same as Fig. 12 but for the $\delta\left\langle r^{2}\right\rangle^{N, 82}$ values of the $\operatorname{Sn}(Z=50)$, Te $(Z=52)$, Xe $(Z=54)$, Ba $(Z=56)$, Ce $(Z=58), \mathrm{Nd}(Z=60), \mathrm{Sm}(Z=52)$, and $\mathrm{Gd}(Z=54)$ isotopes. The experimental data are taken from Ref. [2].

tions: it becomes the lowest in energy in the calculations with $\mathrm{SkM}^{*}$ for $N=34-44$, with UNEDF1 and SLy4 at $N=38$ and with UNEDF0 at $N=38-40$ (see Fig. 20). Similar to our results, the Skyrme calculations for this minimum somewhat overestimate experimental $\delta\left\langle r^{2}\right\rangle^{N, 50}$ values. An excited prolate minimum exists also in PECs of the $\mathrm{Kr}$ isotopes in the CDFT calculations: its deformation drifts from $\beta_{2} \approx 0.45$ for $N=40$ and 42 down to $\beta_{2} \approx 0.25$ for $N=34$ and 36 (see Fig. 17 in the supplemental material). This drift explains the experimentally observed decrease of $\delta\left\langle r^{2}\right\rangle^{N, 50}$ on going from $N=40$ to $N=36$ [see 18(b)].

Note that the PECs obtained for the $\mathrm{Kr}$ and $\mathrm{Sr}$ isotopes in the Gogny DFT calculations with the D1S force (see Ref. [73]) are very similar to those obtained in our calculations. The inclusion of the correlations beyond mean-field within the framework of a five-dimensional collective quadrupole Hamiltonian based on the Gogny DFT allows to improve the description of charge radii in the $N<50 \mathrm{Sr}$ isotopes (see the discussion of Fig. 7 in Ref. [72]). Considering the similarity of mean-field PECs obtained in the CDFT and Gogny DFT calculations, it is reasonable to expect that the inclusion of the correlations beyond mean-field will also improve the description of charge radii in the $N<50$ nuclei of the $\mathrm{Sr}$ region in the CDFT framework.

Finally, Fig. 19 compares the relative properties of differential charge radii of the $\mathrm{Kr}, \mathrm{Sr}$, and Mo isotopic chains obtained in the calculations with the employed functionals. One can see that the clustering of these radii seen for $N=50-58$ in the experiment is reasonably well reproduced in the model calculations.

\section{THE CHARGE RADII IN THE ISOTOPIC CHAINS OF THE CA REGION}

The Ca isotopes have been in the focus of extensive experimental and theoretical studies over the years. In the context of the studies of charge radii, there are two puzzling features of these isotopes, namely, (i) almost exactly the same charge radii of the ${ }^{40,48} \mathrm{Ca}$ isotopes and (ii) a large and unexpected increase of charge radii in neutron-rich beyond $N=28$ nuclei (see Ref. [11]).

First, let us consider the similarity of the charge radii in the ${ }^{40,48} \mathrm{Ca}$ nuclei. These two nuclei are doubly magic with a proton shell closure at $Z=20$ and the neutron shell closures at $N=20$ and 28 , respectively (see Fig. 21 ). As a consequence, proton and neutron pairings are expected either to collapse or to be extremely weak and thus, these two nuclei are ideal candidates for testing of the particle-hole channel of DFTs, underlying EDFs and their isovector dependencies. This is because the theoretical results will not be polluted by the uncertainties in the treatment of pairing. In addition, the PECs presented in Figs. 22(e) and (i) of the supplemental material indicate extreme localization of the ground states of these two nuclei at spherical shape with little or no expected impact from beyond mean-field effects; these features also do not depend on the CEDF.

Table IV and Fig. 25 present the summary of published 


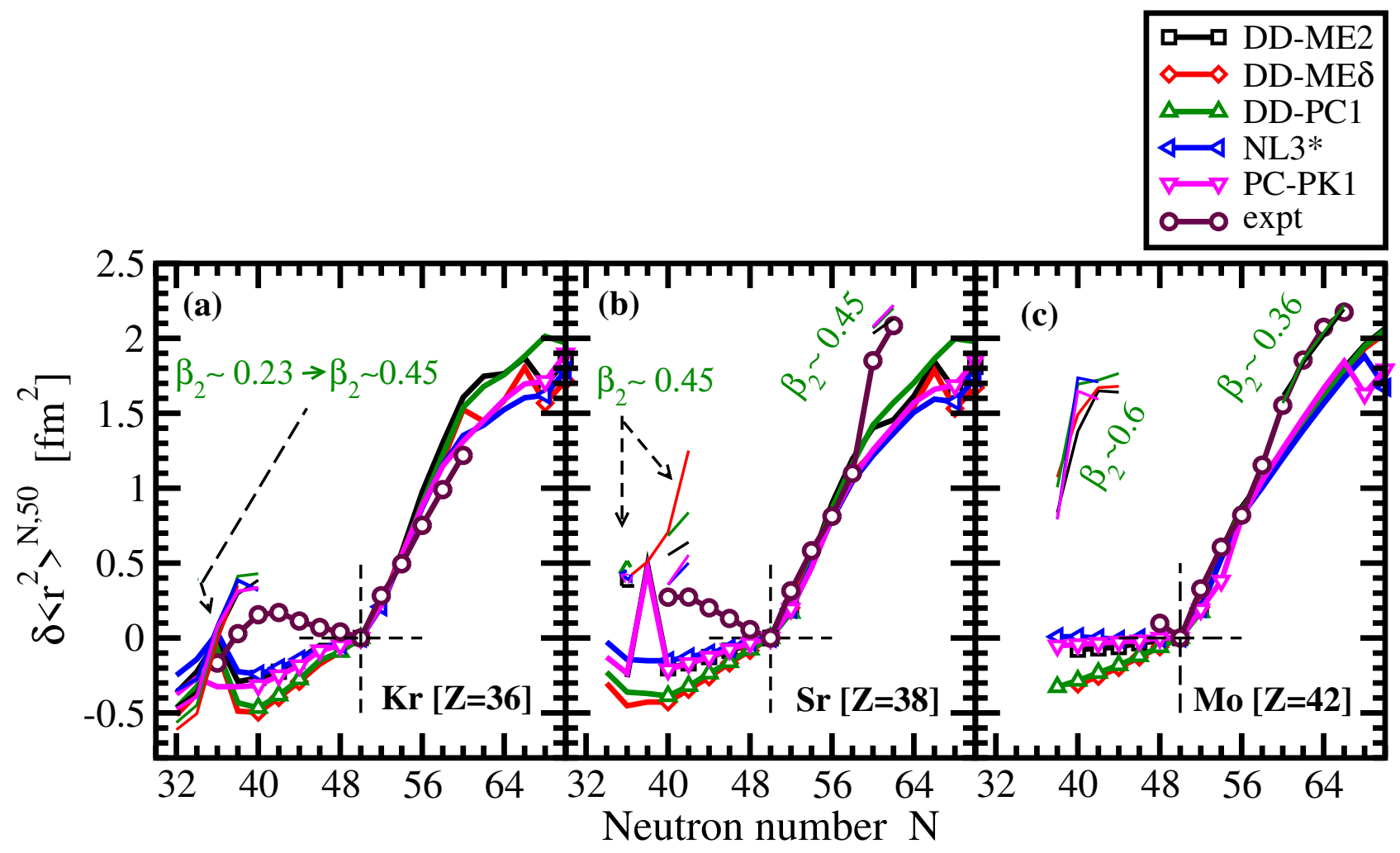

FIG. 18. The $\delta\left\langle r^{2}\right\rangle^{N, 50}$ values of the isotopic chains in the Sr region. The calculated charge radii in excited prolate minima are shown by the thin lines without symbols. The typical deformation in these minima are indicated.

and newly calculated $\delta\left\langle r^{2}\right\rangle^{20,28}$ values for the $\mathrm{Ca}$ isotopes and their connections with nuclear matter properties of the functionals. One can see that $\delta\left\langle r^{2}\right\rangle^{28,20} \approx 0$ is produced by the functionals (such as NL3) characterized by large values of the symmetry energy $J \approx 40 \mathrm{MeV}$ and its slope $L_{0} \approx 110 \mathrm{MeV}$ while the large negative values of $\delta\left\langle r^{2}\right\rangle^{20,28}$ are produced by the CEDFs with low values of $J$ and $L_{0}$. The latter feature is also seen for traditional non-relativistic Skyrme EDFs such as SkM*, SkP, SLy4, SV-min, UNEDF0 and UNEDF1 which are characterized by low values of the symmetry energy $J \approx 32$ $\mathrm{MeV}$ and its slope $L_{0} \approx 50 \mathrm{MeV}$ (see Fig. 24) ${ }^{10}$. It is not likely that the fact that $\delta\left\langle r^{2}\right\rangle^{28,20} \approx 0$ in the Ca isotopes can be related to the details of the single-particle spectra since they are very similar (especially for the proton subsystem) for the functionals which provide different predictions for $\delta\left\langle r^{2}\right\rangle^{28,20}$ (see Fig. 21).

Although there are the correlations between nuclear matter properties and the $\delta\left\langle r^{2}\right\rangle^{20,28}$ values in the Ca isotopes, other factors contribute to experimental $\delta\left\langle r^{2}\right\rangle^{20,28} \approx 0$ value.

10 The Fayans functionals of Ref. [31] are also able to describe $\delta\left\langle r^{2}\right\rangle^{28,20} \approx 0$ but they are specifically designed for that by the use of experimental value of $\delta\left\langle r^{2}\right\rangle^{28,20}$ in the fitting protocol.
First, non-relativistic HFB calculations of Ref. [27] with the semi-realistic M3Y-P6a interaction can reproduce $\delta\left\langle r^{2}\right\rangle^{28,20}$ and $\delta\left\langle r^{2}\right\rangle^{32,28}$ values in the $\mathrm{Ca}$ isotopes: this is attributed to the density dependence of the three-nucleon spin-orbit interaction and its impact on the density distributions of specific single-particle orbitals. However, these calculations fail to reproduce the inverted parabola-like behavior of differential charge radii for $N=22-26$.

Second, it is necessary to recognize that the functionals used in the present paper have been fitted in a time when the importance of the fine structure in the charge radii and alternative mechanisms contributing to them have not been completely recognized. As a result, an approximate Eq. (5) has been used for charge radii in the absolute majority of the publications within the CDFT framework. However, a more general expression for a charge radius in the CDFT is given by $[80,81]$

$$
r_{c h}^{2}=\left\langle r_{p}^{2}\right\rangle+r_{p}^{2}+\left\langle r_{p}^{2}\right\rangle_{S O}+\frac{N}{Z}\left(r_{n}^{2}+\left\langle r_{n}^{2}\right\rangle_{S O}\right)
$$

where $\left\langle r_{p}^{2}>\right.$ stands for proton mean square point radius, $r_{p}$ and $r_{n}$ for single proton and neutron radii, respectively, and $\left\langle r_{p}^{2}\right\rangle_{S O}$ and $\left\langle r_{n}^{2}\right\rangle_{S O}$ for proton and neutron spin-orbit contributions to the charge radius. So, Eq. (5) takes into account only the first two terms of this general expression.

It turns out that Eq. (5) is a quite reasonable approx- 


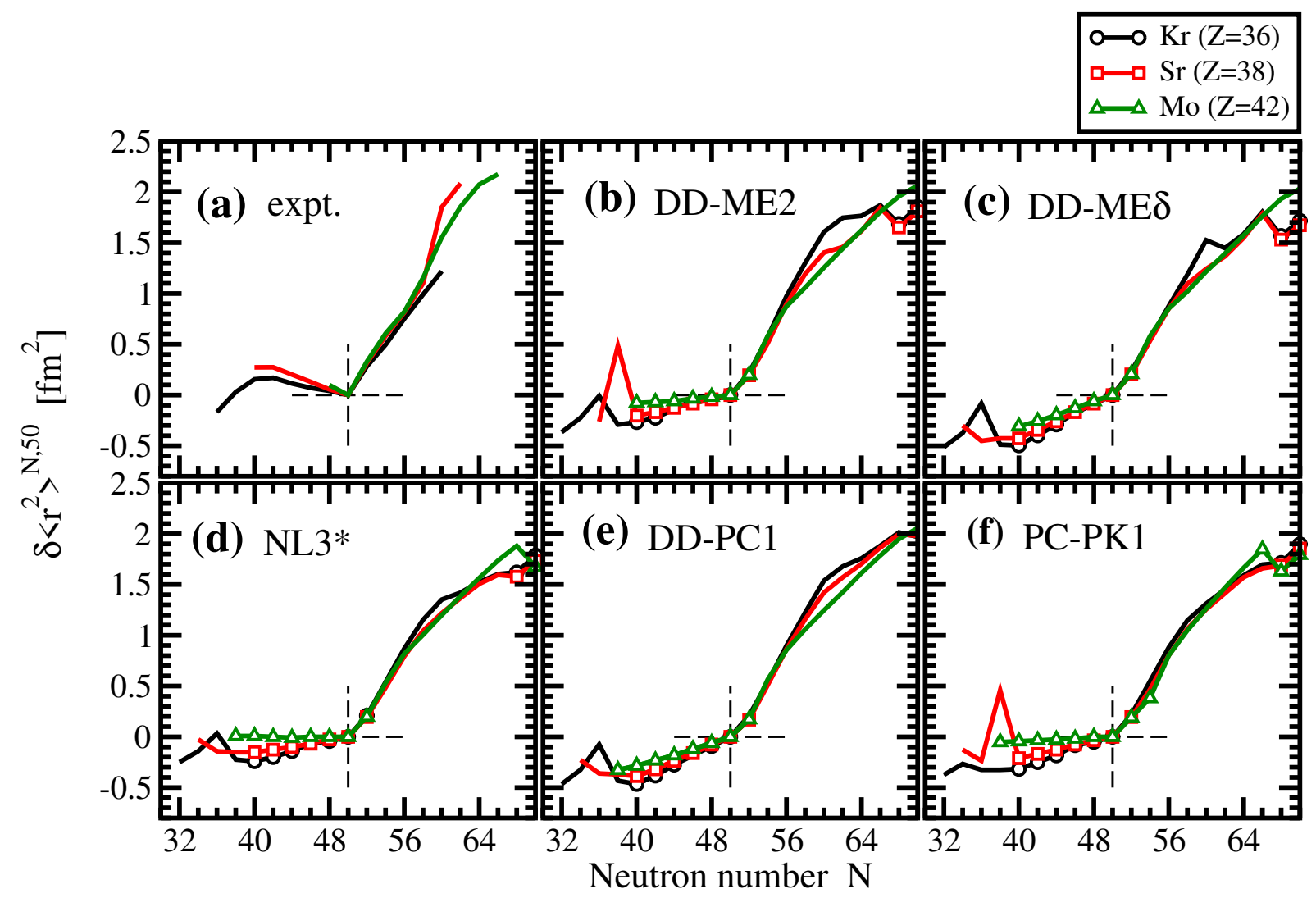

FIG. 19. The same as Fig. 12 but for the $\delta\left\langle r^{2}\right\rangle^{N, 50}$ values of the $\operatorname{Kr}(Z=36)$, $\operatorname{Sr}(Z=38)$, and Mo $(Z=42)$ isotopic chains.

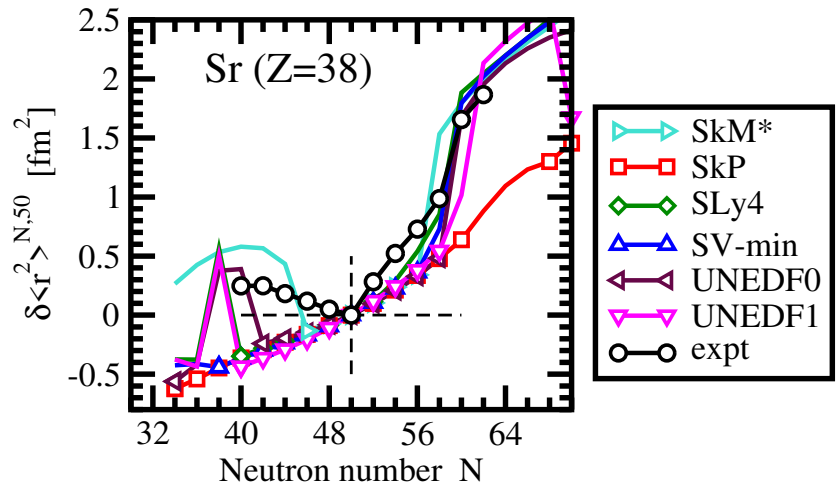

FIG. 20. The $\delta\left\langle r^{2}\right\rangle^{N, 50}$ values for the $\operatorname{Sr}(Z=38)$ isotopes. The experimental data are taken from Ref. [2] and the results of the Skyrme DFT calculations from Mass Explorer [37].

imation to Eq. (10) for medium and heavy mass nuclei especially for differential charge radii. This is because the spin-orbit contribution to charge radii decreases with increasing the mass of nuclei and it almost does not depend on the CEDF. These features are illustrated in Table II of Ref. [80]. Since the calculations of this reference are restricted to spherical shape and neglect pairing correlations, the values quoted for spin-orbit contribution to charge radii in this table for non-doubly magic nuclei

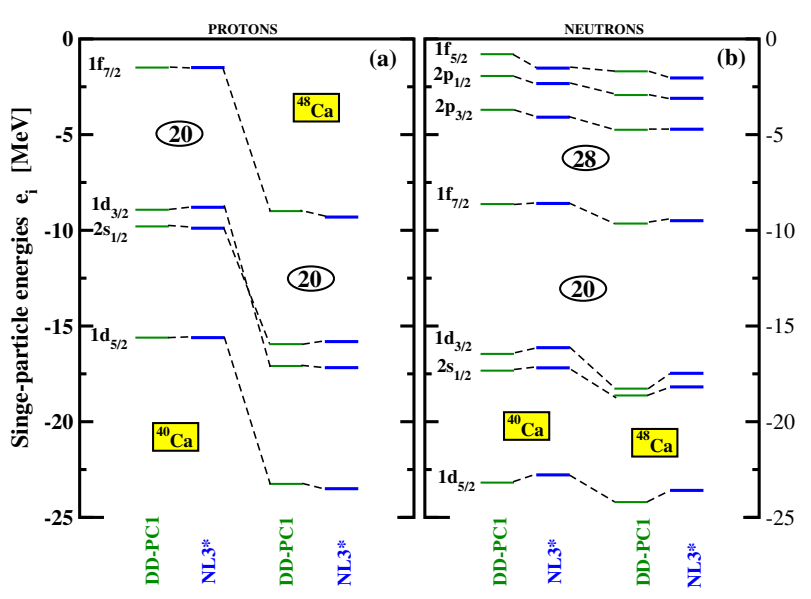

FIG. 21. The same as Fig. 1 but for the ${ }^{40,48} \mathrm{Ca}$ isotopes.

have to be considered as an upper limit. This is because deformation and pairing give rise to the fragmentation of the spin-orbit strength which results in a smoothing of the spin-orbit correction to charge radii as a function of particle number [82]. In addition, for existing experimental data the range of the variation of the $\frac{N}{Z} r_{n}^{2}$ term is significantly smaller in medium and heavy mass nuclei as compared with light nuclei and its contribution to the differential charge radii is cancelled to a large degree. 
TABLE IV. The experimental and calculated $\delta\left\langle r^{2}\right\rangle^{28,20}$ values [in $\left.\mathrm{fm}^{2}\right]$ of the $\mathrm{Ca}(Z=20)$ isotopes and their connection to the nuclear matter properties (such as symmetry energy $J$ and its slope $L_{0}$ ) for the employed CEDFs. "N/A" means that the data are either not applicable or not available. The functionals are arranged in such a way that the calculated $\delta\left\langle r^{2}\right\rangle^{28,20}$ values decrease. References for either the functionals or for related results are shown in the first column.

\begin{tabular}{l|c|c|c|}
\hline \hline CEDF & $\delta\left\langle r^{2}\right\rangle^{28,20}\left[\mathrm{fm}^{2}\right]$ & $J[\mathrm{MeV}]$ & $L_{0}[\mathrm{MeV}]$ \\
\hline exper. [11] & -0.001 & N $/ \mathrm{A}$ & N/A \\
NL-IT [5] & $\approx 0.06$ & 39.4 & N/A \\
NL-SH [74] & 0.040 & 36.13 & 113.68 \\
NL5(E) [75] & 0.031 & 38.93 & 124.96 \\
NL5(D) [75] & 0.003 & 38.87 & 123.98 \\
NL1 [76] & -0.006 & 43.46 & 140.07 \\
NL-I [5] & $\approx-0.01$ & 39.7 & N /A \\
NL3 [77] & -0.014 & 37.40 & 118.53 \\
NL-Z [5] & -0.015 & 41.72 & 133.91 \\
NL3* [44] & -0.028 & 38.68 & 122.60 \\
DD-MEX [78] & -0.056 & 32.87 & 47.81 \\
NL5(A) [75] & -0.088 & 34.92 & 108.85 \\
NL5(C) [75] & -0.092 & 35.925 & 112.31 \\
NL5(B) [75] & -0.094 & 34.92 & 108.33 \\
PCPK1 [48] & -0.098 & 35.60 & 113.00 \\
DDME2 [46] & -0.111 & 32.40 & 49.40 \\
DD-PCX [39] & -0.178 & 31.12 & 46.32 \\
DD-PC1 [45] & -0.229 & 33.00 & 68.40 \\
DDME $\delta[47]$ & -0.296 & 32.35 & 52.90 \\
\hline
\end{tabular}

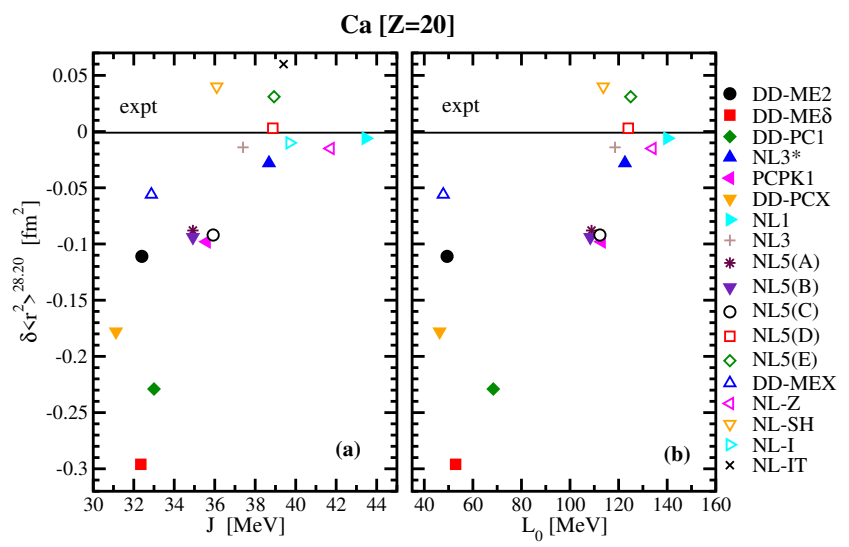

FIG. 22. The relations between the calculated $\delta\left\langle r^{2}\right\rangle^{28,20}$ values in the $\mathrm{Ca}$ isotopes and the symmetry energy $J$ and its slope $L_{0}$ of employed functionals. See Table IV for additional details.

However, the last two terms of Eq. (10) are important in light nuclei (see Refs. [80, 81]). For example, when these terms are taken into account the differential charge radius $\delta\left\langle r^{2}\right\rangle^{20,28}$ for the $\mathrm{Ca}$ isotopes changes from $-0.013 \mathrm{fm}^{2}$ to $+0.164 \mathrm{fm}^{2}$ in the NL3 CEDF and from $-0.07 \mathrm{fm}^{2}$ to $+0.108 \mathrm{fm}^{2}$ in the NLSH one (see Table 1 in Ref. [81]) moving away from experimental value of $-0.001 \mathrm{fm}^{2}$. Let us assume a similar range of correc-
TABLE V. The experimental and calculated $\delta\left\langle r^{2}\right\rangle^{32,28}$ values [in $\left.\mathrm{fm}^{2}\right]$ of the $\mathrm{Ca}(Z=20)$ and $\mathrm{Fe}(Z=26)$ isotopes.

\begin{tabular}{l|c|c}
\hline \hline CEDF & $\mathrm{Ca}$ & $\mathrm{Fe}$ \\
\hline exper. & 0.530 & 0.606 \\
DDME2 & 0.407 & 0.670 \\
DDME $\delta$ & 0.377 & 0.633 \\
DD-PC1 & 0.302 & 0.591 \\
NL3 ${ }^{*}$ & 0.336 & 0.645 \\
PCPK1 & 0.317 & 0.697 \\
\hline
\end{tabular}

tions by these two terms of Eq. (10) for other functionals. Then the accuracy of the description of experimental $\delta\left\langle r^{2}\right\rangle^{20,28} \approx 0$ value after inclusion of these corrections would degrade for the functionals listed in upper part (down to $\mathrm{NL}^{*}$ ) of Table IV, would remain similar for DD-MEX and would improve for the functionals located in the bottom part of Table IV.

An analysis of the contributions of spin-orbit densities and other terms to charge radii has also been performed in the non-relativistic framework (see Refs. [8284]). There are some differences between relativistic and non-relativistic treatments of these terms (see detailed discussion in Ref. [81]), however, in general a comparable modification of charge radii is generated by such terms in non-relativistic DFTs. For example, these contributions change $\delta\left\langle r^{2}\right\rangle^{20,28}$ of the Ca isotopes from $-0.198 \mathrm{fm}^{2}$ to $-0.048 \mathrm{fm}^{2}$ (see Table 1 in Ref. [81] and Fig. 1 in Ref. [84] in the Skyrme DFT calculations with the SLy4 functional bringing them closer to experimental data. Similar results are obtained for the Skyrme SV-bas and Fayans $\mathrm{Fy}(\Delta r, \mathrm{HFB})$ functionals in Ref. [82] with spin-orbit density and $\frac{N}{Z} r_{n}^{2}$ term providing approximately $0.1 \mathrm{fm}^{2}$ and $0.04 \mathrm{fm}^{2}$ contributions to the $\delta\left\langle r^{2}\right\rangle^{20,28}$ value, respectively. Assuming that similar corrections appear for other non-relativistic functionals shown in Fig. 24, it is clear that their addition will improve the description of experimental $\delta\left\langle r^{2}\right\rangle^{20,28}$ value in these functionals. However, their addition do not allow either to reproduce the inverted parabola-like behavior of differential charge radii for $N=22-26$ or improve the description of the large $\delta\left\langle r^{2}\right\rangle^{32,28}$ experimental value in the Ca isotopes (see Fig. 1 in Ref. [84]). The latter is because spin-orbit densities do not modify substantially the calculated $\delta\left\langle r^{2}\right\rangle^{32,28}$ value.

Since the experimental data on charge radii is included in the fitting protocols (which rely on Eq. (5) for the definition of charge radii) of the CEDFs employed in the present paper, they partially include the corrections provided by additional terms of Eq. (10). Thus, to avoid double counting of these corrections, new fits of CEDFs with charge radii defined by Eq. (10) are needed. They will provide a more consistent (and, hopefully, more accurate) description of charge radii. However, in the context of the present study with the employed functionals 


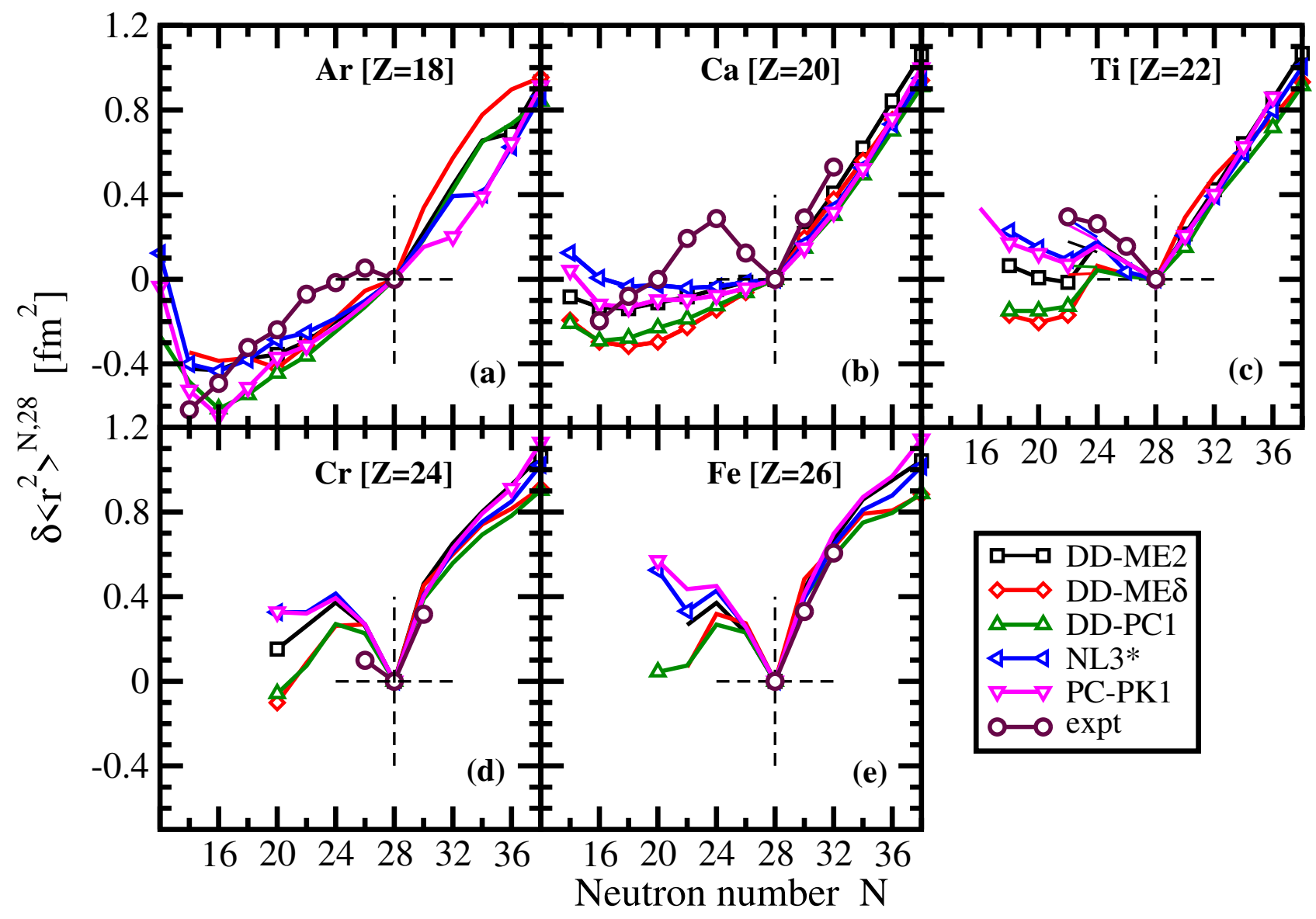

FIG. 23. The $\delta\left\langle r^{2}\right\rangle^{N, 28}$ values of the isotopic chains in the Ca region. The experimental data for the Ar, Ti, Cr and Fe isotopes are taken from Ref. [2]. The experimental data for the Ca isotopes are mostly taken from Ref. [11] while that for the ${ }^{39,41} \mathrm{Ca}$ isotopes from Ref. [79] and for ${ }^{36,37,38}$ Ca from Ref. [12].

one can conclude that for a given isotopic chain the relative properties of charge radii and differential charge radii provided by two CEDFs should not be very much affected by these corrections. This is because existing studies indicate their weak dependence on the functional. On the other hand, the calculated absolute values of these radii are expected to be partially affected by these corrections and this fact is taken into account in further discussion. However, this effect is expected to be reasonably small for the calculated differential charge radii of isotopic chains in the experimentally available range in neutron number if this range is rather short (see Ref. [82]). These are Ti, $\mathrm{Cr}$ and Fe isotopic chains (see, for example, Fig. 23).

These new functionals are also needed to answer the question on whether $\delta\left\langle r^{2}\right\rangle^{20,28} \approx 0$ of the $\mathrm{Ca}$ isotopes can provide a meaningful constraint on nuclear matter properties. The discussion provided above on that issue is not conclusive. The analysis of proton density distributions determined by electron scattering and the neutron density distributions determined by proton elastic scattering in the ${ }^{40,48} \mathrm{Ca}$ nuclei supplemented by the studies within Skyrme DFT and CDFT support low values of $J \approx 28 \mathrm{MeV}$ and $L=28-50 \mathrm{MeV}$ [85]. In contrast, the recent PREX-II measurements of the neutron skin in ${ }^{208} \mathrm{~Pb}$ [61] imply a stiff equation of state with large $J=38.1 \pm 4.7$ and $L_{0}=106 \pm 37 \mathrm{MeV}$ values [86].

The calculated and experimental differential charge radii are compared in Figs. 23 and 25. One can see that all employed CEDFs fail to describe the evolution of differential charge radii in the $\mathrm{Ca}$ isotopes in the neutron number range $N=16-28$ and especially the peak at $N=24$ [see Fig. 23(b)]. However, the same problem exists also in all non-relativistic Skyrme EDFs (see Fig. 24). As discussed above, the accounting of spin-orbit densities and the $\frac{N}{Z}\left\langle r_{n}^{2}\right\rangle$ term in Eq. (10) can somewhat modify this situation but in no way will it correct the problem with the description of the peak at $N=24$. The analysis of the occupation probabilities indicates that mostly neutron $1 f_{7 / 2}$ states are occupied in the transition from ${ }^{40} \mathrm{Ca}$ to ${ }^{48} \mathrm{Ca}$, and this leads either to a linear increase or nearly constant differential charge radii in conventional functionals. This figure also indicates that the inverted parabola-like behavior of differential charge radii in the $N=20-28$ isotopes is reproduced on average only in the Fayans $\mathrm{Fy}(\Delta \mathrm{r})$ functional which includes gradients both in surface and pairing terms and was fitted to ex- 


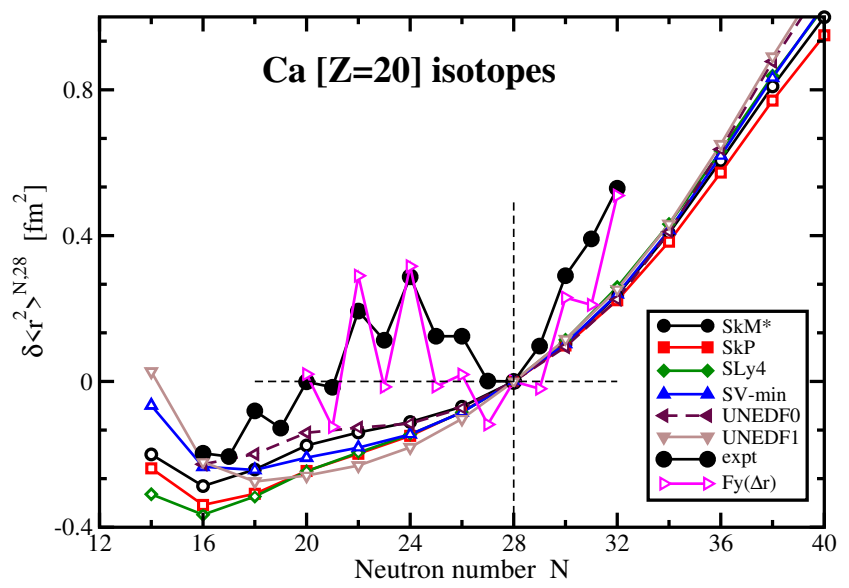

FIG. 24. The $\delta\left\langle r^{2}\right\rangle^{N, 28}$ values of the Ca $(Z=20)$ isotopes relative to the $N=28$ isotope. The experimental data for the Ca isotopes are mostly taken from Ref. [11] while that for the ${ }^{39,41} \mathrm{Ca}$ isotopes from Ref. [79] and for ${ }^{36,37,38} \mathrm{Ca}$ from Ref. [12]. The results of the Skyrme DFT calculations are taken from Mass Explorer at FRIB [37]. The results of the Fayans $\mathrm{Fy}(\Delta \mathrm{r}, \mathrm{HFB})$ functional are taken from Fig. 4 of Ref. [31].

perimental (absolute and relative) data on charge radii in ${ }^{40,44,48} \mathrm{Ca}$ in Ref. [31].

The rise in the differential charge radii above the $N=28$ shell closure is underestimated in the model calculations (see Fig. 23(b) and Table V). The nonrelativistic results of Ref. [84] indicate that the $\delta\left\langle r^{2}\right\rangle^{32,28}$ value is only moderately affected by spin-orbit densities. If that result holds also in the CDFT, then the best reproduction of experimental $\delta\left\langle r^{2}\right\rangle^{28,32}$ values is achieved by the DD-ME2 functional which underestimates it only by $23 \%$ (see Table V). Note that the problem with the description of the rise in charge radii in the $\mathrm{Ca}$ isotopes above $N=28$ also exist in non-relativistic Skyrme and Gogny calculations and in non-relativistic ab initio calculations (see, for example, Fig. 24 in the present paper and Fig. 3 in Ref. [11]).

The slope of the experimental $\delta\left\langle r^{2}\right\rangle^{N, 28}$ curve for the $\operatorname{Ar}(Z=18)$ isotopes is best reproduced in the calculations with the DD-PC1 and PC-PK1 functionals [see Fig. 23(a)]. However, even these functionals underestimate the experimental data by $\approx 0.2 \mathrm{fm}^{2}$. Based on available estimates in this mass region (see Refs. [80$82,84]$ ), the contribution of spin-orbit densities to the differential charge is below this value. Note that in the calculations, many of the Ar isotopes are oblate in their ground states (see Fig. 18 in the supplemental material) and that the PECs of almost all Ar isotopes are extremely soft in quadrupole deformation (see Fig. 23 in the supplemental material). In such a situation, the correlations beyond mean-field are expected to play an important role.

The differential charge radii in the $\mathrm{Ti}(Z=22)$ isotopes are gradually decreasing from $\delta\left\langle r^{2}\right\rangle^{N, 28} \approx 0.3 \mathrm{fm}^{2}$ at $N=22$ down to zero at $N=28$ (see Fig. $23(\mathrm{c})$ ). This trend is reasonably well reproduced for $N=24-28$ by the NL3*, PC-PK1, and DD-ME2 functionals. This is due to two facts. First, the $N=24$ and 26 isotopes are prolate in the calculations with $\beta_{2} \approx 0.15$ (see Fig. 18 in the supplemental material), so their charge radii are larger than those at the spherical shape. Second, these are two functionals that reproduce reasonably well the near equality of charge radii in ${ }^{40,48} \mathrm{Ca}$ (see Table IV). Other functionals (DD-PC1 and DD-ME $\delta$ ), which cannot reproduce this feature, also fail to describe the evolution of charge radii in the $\mathrm{Ti}$ isotopes. Note that in the calculations, the PEC of the $\mathrm{N}=22,24$, and 26 isotopes are soft in quadrupole deformation, which suggests that beyond mean-field effects could play an important role in the reproduction of experimental data. Assuming that these effects could lead to the creation of a prolate minimum with $\beta_{2} \approx 0.2$ in the $N=22$ isotope, this would also allow explaining the charge radius of this isotope in the calculations with PC-PK1, DD-ME2, and NL3* (see results presented by thin solid magenta, black and blue lines in Fig. 23(c)).

The experimental differential charge radii of the $\mathrm{Cr}$ $(Z=24)$ isotopes show asymmetric parabola-like features at $N=26-30$ with the minimum at $N=28$ [see Fig. 23(d)]. The experimental $\delta\left\langle r^{2}\right\rangle^{26,28}$ value is overestimated by a factor of approximately 1.5 in all calculations. Note that in the model calculations, the $N=26$ and $N=30$ isotopes are prolate with $\beta_{2} \approx 0.22$, while the $N=28$ isotope is spherical (see Fig. 18 in the supplemental material). The PECs of these isotopes are soft in quadrupole deformation (see Fig. 19 in the supplemental material), so the ground-state properties of these nuclei could be affected somewhat by beyond mean-field correlations.

The experimental differential charge radii of the $\mathrm{Fe}$ $(Z=26)$ isotopes are available for $N=28-32$ [see Fig. 23(e)]. The large increase in $\delta\left\langle r^{2}\right\rangle^{N, 28}$ observed in the experiment above $N=28$ is rather well reproduced in model calculations (see above mentioned figure and Table $\mathrm{V})$. The $N=28$ isotope is spherical or quasi-spherical in model calculations, while the $N=30$ and 32 isotopes are prolate with a quadrupole deformation of $\beta_{2} \approx 0.23$ (see Figs. 18 and 19 in the supplemental material).

It is clear that the description of differential charge radii in the Ca region represents a case of the "hit-ormiss" situation when some data are rather well described while others are difficult to reproduce. This is especially the case for the relative properties of differential charge radii of different isotopic chains. Figure 25 shows that it is more difficult to reproduce them in the Ca region as compared with other regions. It is expected that this conclusion will not be affected by the inclusion of the spin-orbit densities or the $\frac{N}{Z}\left\langle r_{n}^{2}\right\rangle$ term.

It is interesting that the $N=22-28 \mathrm{Ca}$ and Ti isotopes show very similar trends in differential charge radii [see Fig. 25(a)]. It is also likely that the Cr isotopes will follow the same trend if experimental data are extended 
to lower neutron numbers. The challenge here lies in the fact that in the model calculations, the $N=22-26$ isotopes are prolate and spherical in the $\mathrm{Ti}$ and $\mathrm{Ca}$ isotopic chains, respectively (see Fig. 18 in the supplemental material). In contrast to the experiment, this suggests a quite substantial difference in charge radii. Indeed, the model calculations with some functionals can reproduce the data in the $\mathrm{Ti}$ isotopes but fail to do that in the $\mathrm{Ca}$ ones.

One possible exit from this contradiction is the possibility that the $N=22-26 \mathrm{Ca}$ isotopes are much softer in quadrupole deformation as compared with the suggestion provided by PECs obtained at the mean-field level (see Fig. 22 of the supplemental material). The low excitation energy of the superdeformed (SD) band in ${ }^{40} \mathrm{Ca}$ (see Ref. [87]), which is the lowest amongst all SD bands in the nuclear chart, may point to a substantial softness of the $\mathrm{Ca}$ isotopes. To our knowledge, mean-field calculations substantially overestimate the excitation energy of this SD band and thus, quite likely, predict stiffer Ca isotopes. The enhanced influence of the correlations beyond meanfield on the charge radii of the $\mathrm{Ca}$ isotopes as compared with the $\mathrm{Sn}$ and $\mathrm{Pb}$ isotopes has already been pointed in Refs. [5, 30]. However, its magnitude and neutron number dependence are not sufficient to reproduce the peak in the differential charge radii at $N=24$. If the meanfield PECs are softened (especially for the $N \neq 20,28$ isotopes) then the correlations beyond mean-field could be enhanced and maybe the experimental peak in $\delta\left\langle r^{2}\right\rangle^{N, 20}$ at $N=24$ could be reproduced. In contrast, the analysis of Ref. [88] partially based on the QRPA calculations indicates that the ground state correlations associated with the surface modes of the Ca isotopes are important and that they qualitatively explain the observed inverted parabola-like behavior of differential charge radii with neutron number for $N=20-28$. However, these calculations rely on a number assumptions which have to be verified in more microscopic calculations. All these calculations point to potential limitations of the mean field approximation in the description of the ground state properties of light nuclei such as the $\mathrm{Ca}$ isotopes and the need to include correlations beyond mean field.

\section{ODD-EVEN STAGGERING IN CHARGE RADII}

The compilation of all available experimental data on OES in charge radii is presented in Figs. 26 and 27. In most of the cases, the charge radius of an odd- $N$ nucleus is smaller than the average of its even- $N$ neighbors. This corresponds to positive and negative values of the $\Delta\left\langle r^{2}\right\rangle^{(3)}(N)$ indicators at odd- and even- $N$ values, respectively. However, in approximately $25 \%$ of the cases (indicated by dashed circles in Figs. 26 and 27), this order is inverted. Then we speak about inverted OES in charge radii. The origin of this inversion depends on the neutron number.
The full or near-complete collapse of neutron pairing at magic neutron shell closures at $N=28$ in the $\mathrm{Ca}$ isotopes [Fig. 26(c)], at $N=50$ in the Kr, Sr [Figs. 26(e) and (f)] and Rb [Fig. 27(c)], at $N=82$ in the Sn, Ba, Nd, and Sm [Figs. 26(h), (j), (k) and (l)], Cs and Eu [Figs. 27(e) and (f)] isotopes, at $N=126$ in the $\mathrm{Pb}$ [Fig. 26(r)] and $\mathrm{Bi}$ [Fig. 27(n)] is one of such sources of the inversion of OES in charge radii. Note that this kind of inversion is mostly localized at neutron numbers corresponding to the shell closures in these isotopic chains.

The transition from spherical or quasi-spherical nuclei to deformed ones taking place with increasing neutron number at $N \approx 88$ triggers the inversion of OES in charge radii of the $\mathrm{Dy}, \mathrm{Tb}, \mathrm{Eu}$, and $\mathrm{Tm}$ isotopic chains [see Fig. 26(m) and Fig. 27(g), (f) and (i)]. A similar transition at $N \approx 58$ is responsible for the inversion of OES in the Rb isotopic chain [see Fig. 27(c)]. Note that not in all cases this kind of transition triggers the inversion of OES: the magnitude of OES in charge radii is simply increased in the vicinity of these neutron numbers as compared with the ones for lower/higher $N$ values in isotopic chains of the Kr, Sr, Sm, and Ho [Fig. 26(e), (f) and (m) and Fig. $27(\mathrm{~g})]$.

In addition, several other mechanisms of OES in charge radii and its inversion have been suggested earlier. They will be discussed below using the results obtained in the CDFT framework. However, the mechanism presented in Sec. IX C is completely new.

\section{A. Shape coexistence as a source of OES in charge radii and its inversion}

Significant odd-even staggering in the $\mathrm{Hg}$ charge radii exists at $N=100-106$ [see Fig. 7 (a) and Fig. 28]. Several scenarios have been suggested for an explanation of this OES (see overviews in Sec. 4.7 of Ref. [69] and in Ref. [89]), but the one which agrees most with experimental data on OES in charge radii was first suggested in Ref. [96]. This paper, together with the analysis in the Skyrme DFT (Ref. [89]) and CDFT (present paper) suggest the following scenario: the even- $N$ isotopes should have a weakly deformed oblate minimum (quasi-spherical in the language of Ref. [69]) while the odd- $N$ nuclei in the region should have large prolate deformations. Two such minima (oblate with $\beta_{2} \approx-0.15$ and prolate with $\beta_{2} \approx 0.3$ ) coexists in the isotopes of interest (see Fig. 11). Note that the latter values are close to experimental estimates of the deformations in ${ }^{181,183,185} \mathrm{Hg}$ (see Ref. [69]). Under such a scenario the evolution of the $\delta\left\langle r^{2}\right\rangle^{N, 126}$ values for even- $N$ numbers is reasonably well described, especially with the NL3* and PC-PK1 functionals (see Fig. 28). In addition, the magnitude of OES (as the difference of the charge radii in prolate and oblate minima) is not far away from experimental values.

The only caveat in this CDFT interpretation is the fact that the prolate minimum is the lowest in energy in the nuclei for which OES in charge radii is observed (see Fig. 


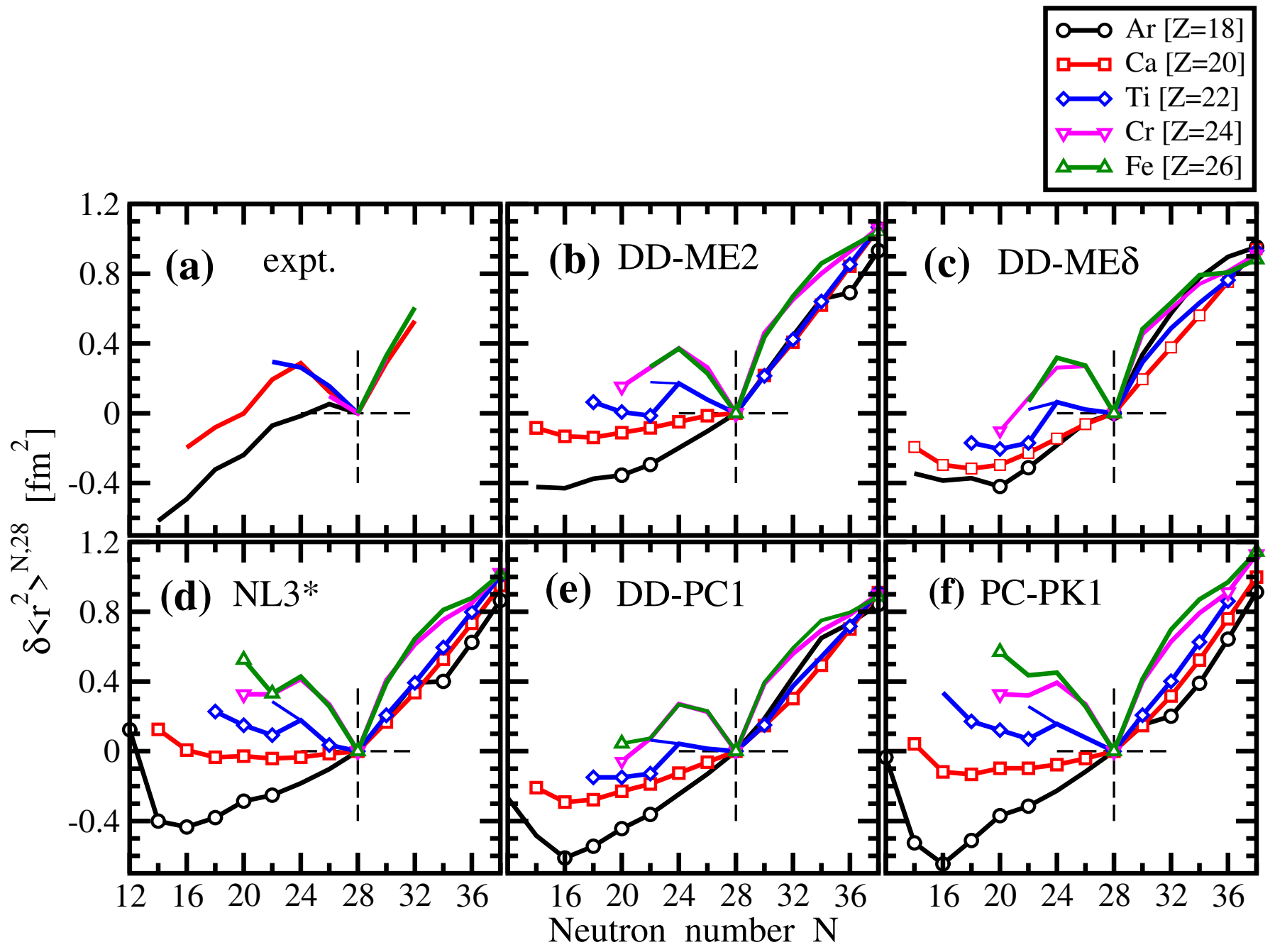

FIG. 25. The same as Fig. 12 but for the $\delta\left\langle r^{2}\right\rangle^{N, 28}$ values of the $\operatorname{Ar}(Z=18)$, Ca $(Z=20)$, Ti $(Z=22)$ and Fe $(Z=26)$ isotopic chains. Thin blue solid line connecting $N=22$ and $N=24$ illustrates the situation which would emerge if the $N=22$ Ti nucleus would be deformed with $\beta_{2}=0.2$ instead of being spherical.

11). However, the oblate minimum is only by approximately $1 \mathrm{MeV}$ higher in energy than the prolate one for most functionals. The only exception is the DD-ME $\delta$ functional, for which this difference is more significant. One should note that this energy difference between the minima is extremely sensitive to the fine details of the functional and that most non-relativistic models also fail to reproduce this difference (see review in Sec. IVD of Ref. [89] and Ref. [97]). Note also that the PECs in $\mathrm{Hg}$ nuclei with $N \leq 100$ show that the oblate minimum becomes the lowest in energy, and the prolate minimum starts to disappear. This is consistent with the disappearance of OES in charge radii seen in the experiment at low $N$ (see Fig. 28).

Since the deformation and thus the charge radii are larger in odd- $N$ isotopes as compared with even- $N$ ones, the OES of charge radii in the light $\mathrm{Hg}$ isotopes is in- verted $^{11}$ [see Fig. 26(q)]. Note that this is the largest OES of charge radii in the whole nuclear chart. A similar, but somewhat smaller, inverted OES is observed in the neighboring Pt (for $N=101-110$ ) [see Fig. 26(p)] and Au (for $N=105-108$ ) [see Fig. 27(l)] isotopic chains. Considering the magnitude of OES as well as its localization in neutron number, it is quite likely that it has the same origin as in the $\mathrm{Hg}$ isotopes. It may be that the small inverted OES seen at $N=101-104$ in the $\mathrm{Pb}$ isotopes [see Fig. 26(r)] has a similar origin.

11 One can easily imagine a situation where the absolute values of deformation (and thus charge radii) are smaller in odd- $N$ isotopes as compared with even- $N$ ones. This will lead to a regular OES. Thus, a sensitive energy balance between two local minima with different deformations and deformation driving properties of the unpaired orbital in the odd- $N$ nucleus will define whether OES is regular or inverted. 

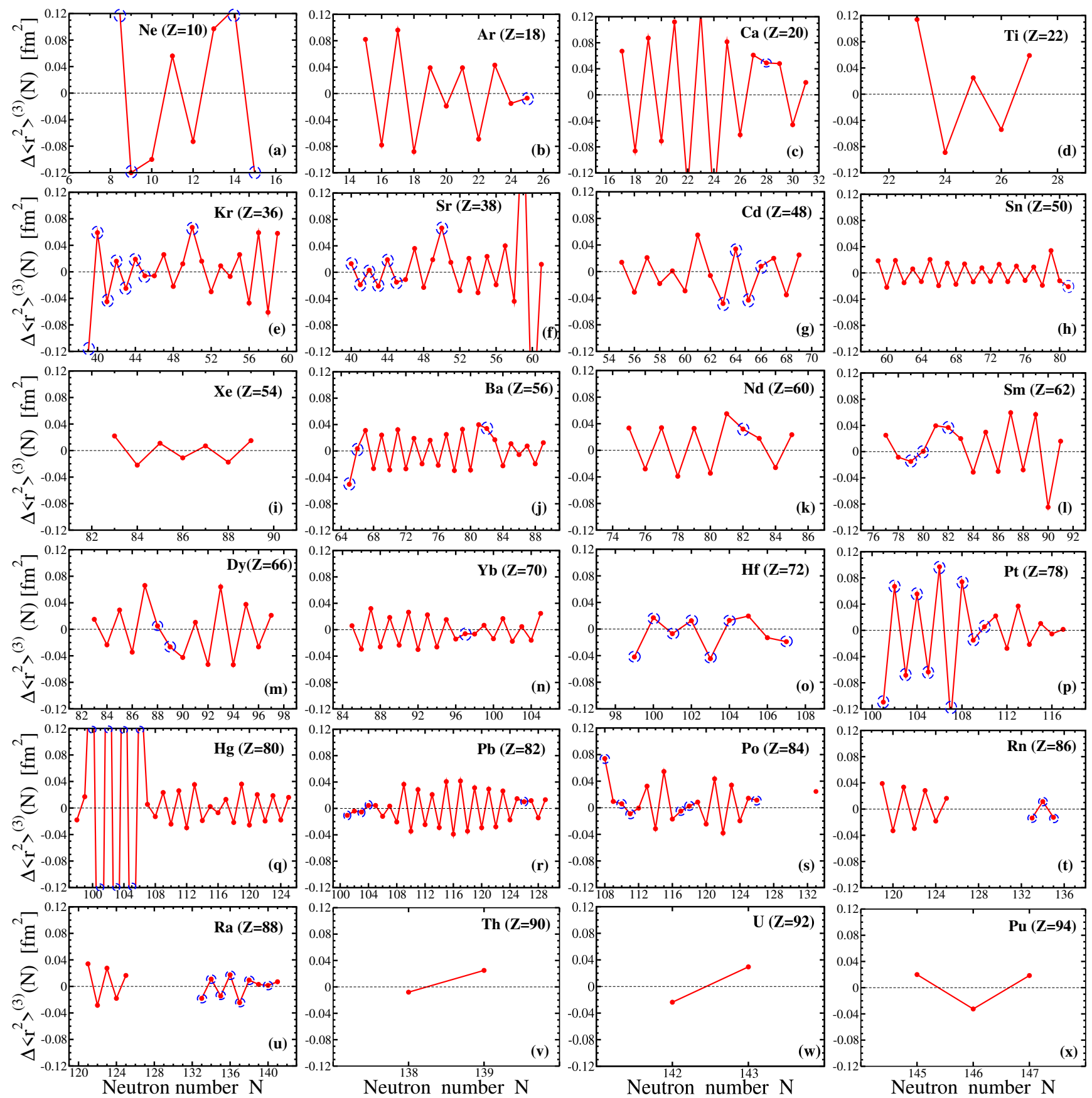

FIG. 26. Experimental OES of charge radii of even- $Z$ nuclei. The data points are encircled by blue dashed circles when they deviate from the regular pattern [regular OES], namely, from $\Delta\left\langle r^{2}\right\rangle^{(3)}(N)>0$ for odd values of $N$ and from $\Delta\left\langle r^{2}\right\rangle^{(3)}(N)<0$ for even values of $N$. These encircled points correspond to inverted OESs. The experimental data are taken from Ref. [2]. Note that, with the exception of the Ca isotopes, we use the same range of $\Delta\left\langle r^{2}\right\rangle^{(3)}$ on the vertical axis of all panels. Until specified otherwise, the experimental data are taken from Ref. [2]. Only for the Ca isotopes they are mostly taken from Ref. [11], from Ref. [79] for ${ }^{39,41} \mathrm{Ca}$ and from Ref. [12] for ${ }^{36,37,38} \mathrm{Ca}$. The experimental data for the radii are taken from Ref. [89] for the Hg isotopes with $N<106$ and from [90,91] for the Po isotopes.

\section{B. Pairing as a source of OES in charge radii}

As illustrated in previous examples, the charge radii are increasing nearly linearly with increasing neutron number when the single-particle states of the same spher- ical neutron subshell (let us call it a $j$-shell) are occupied. This trend is schematically illustrated as a dashed line in Fig. 32. In the calculations with pairing, the blocking effect in odd- $N$ nuclei leads to an additional redistribution of the occupation of the single-particle orbitals 

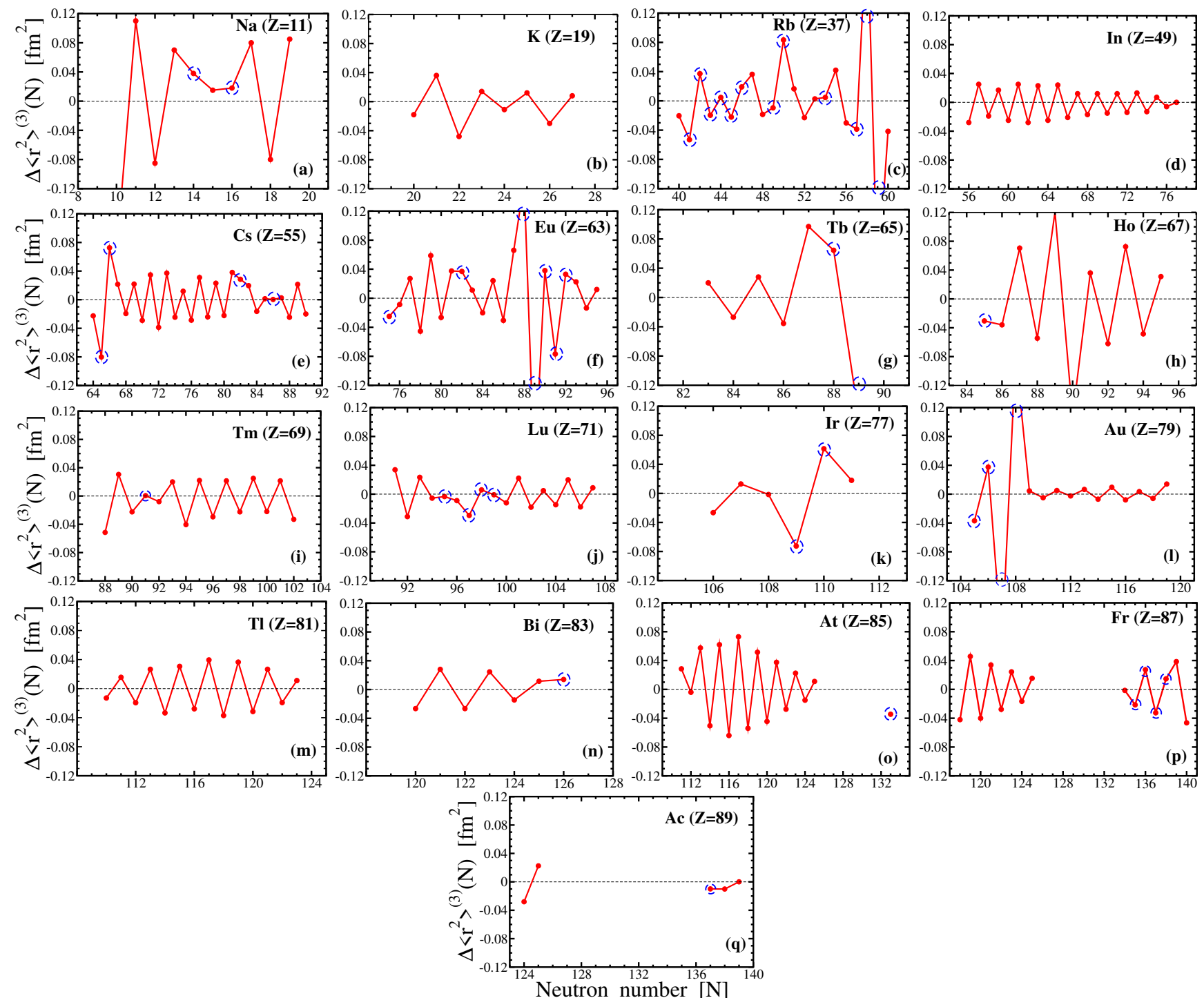

FIG. 27. The same as Fig. 26 but for odd- $Z$ nuclei. The experimental data are taken from Refs. [2] (compilation), [92] (Fr isotopes), [19, 93] (At isotopes) and [94, 95] (Ac isotopes).

and typically to a decrease of the charge radius of the odd- $N$ nucleus below the average given by two even-even neighbors. As a consequence, the increase in charge radii $\Delta\left(r_{c h}\right)_{M F}^{q p}$ on going from even- $N$ to odd- $A$ is typically smaller as compared both with the increase corresponding to a linear increase defined by $r_{c h 1}$ and $r_{c h 3}$ and that obtained in the calculations without pairing (see Fig. 32). This leads to a regular OES in charge radii and, so far, the pairing has been considered to be its dominant source in isotopic chains which do not undergo significant shape changes like those discussed in Sec. IX A (see Refs. $[30,31,69])$.

Let us consider an example of realistic calculations in the $\mathrm{Sn}$ isotopes. In the RHB calculations, two different procedures labeled as LES (lowest in energy solution) and EGS (experimental ground-state) are used for the blocking in odd-A nuclei ${ }^{12}$, and these abbreviations label the results of the respective calculations. In the LES procedure, the lowest in energy configuration is used. It has been applied in all earlier calculations of OES with non-relativistic DFTs [30, 31]. In the EGS procedure, the configuration with the spin and parity of the blocked state corresponding to those of the experimental ground state is employed, although it is not necessarily the lowest in energy.

In Fig. 29 the results of calculations with different functionals are compared with experimental data. One can see that the results of the RHB calculations with the LES procedure significantly underestimate the mag-

\footnotetext{
12 They were first employed in the studies of OES of differential radii in the $\mathrm{Pb}$ and $\mathrm{Hg}$ isotopic chains in Ref. [17].
} 


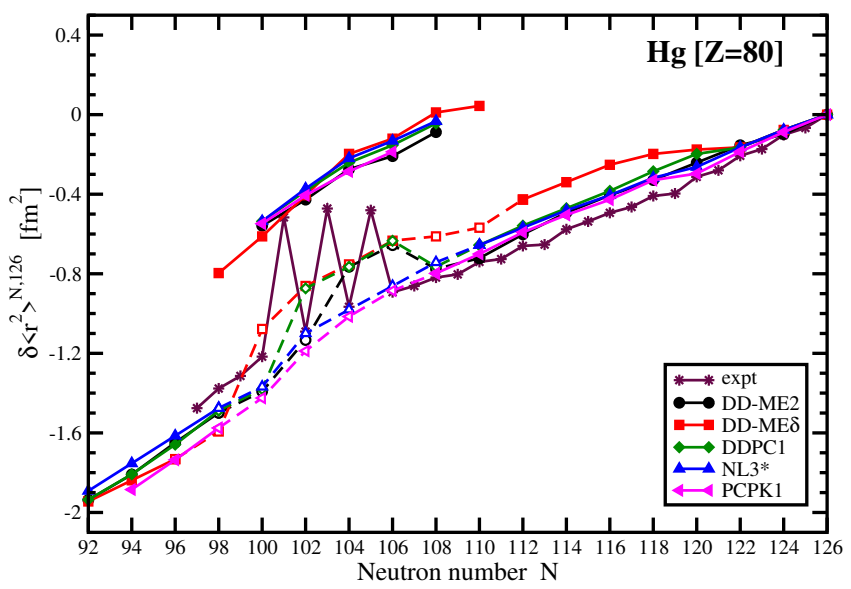

FIG. 28. Calculated and experimental $\delta\left\langle r^{2}\right\rangle^{N, 126}$ values of the $\mathrm{Hg}$ isotopes. The values corresponding to the lowest in energy solutions are shown by solid lines, while those for excited ones by dashed lines.

nitude of experimental OES and occasionally provide a wrong phase of the OES. The use of the EGS procedure significantly improves the description of both the phases and the magnitude of OES, especially for DD$\mathrm{ME} \delta$. However, even in that case, the magnitude of OES is underestimated by a factor of approximately two. This suggests that an important part of physics is still missing; it is addressed in Sec. IX C. Note that the analysis of the $\mathrm{Pb}$ and $\mathrm{Hg}$ isotopic chains performed both in relativistic and non-relativistic frameworks indicates that the EGS procedure is needed for a proper description of OES in charge radii (see Ref. [17]).

A significant underestimate of OES in charge radii is also observed in Skyrme DFT calculations with conventional functionals. The suggested resolution of this problem lies in the use of Fayans functionals which include gradient terms in both surface term and pairing [29, 30]. The latest functional of this type is $\mathrm{Fy}(\Delta r)$ [31]. However, it overestimates the magnitude of OES in the Sn [14] and other isotopic chains [31].

\section{Particle-vibration coupling as a source of OES in charge radii}

There is a principal difference between the ground states in even-even and odd nuclei which is schematically illustrated in Fig. 30 and which has been ignored in the studies of differential charge radii before. It is related to a substantial fragmentation of the wavefunction of the ground states in odd- $A$ nuclei (especially in spherical nuclei) due to the coupling of single-particle motion with phonons [particle-vibration coupling (PVC)]. In even-even nuclei, the correlations beyond mean-field can affect the binding energies and equilibrium deformations [and thus the charge radii] of the ground states, but they do not lead to a significant fragmentation of their wavefunctions in the nuclei with stiff parabola-like PECs (such as $\mathrm{Pb}$ and $\mathrm{Sn}$ isotopes [see, for example, Fig. 12 in the supplemental material]). Indeed, such correlations are rather small in the ground states of the even-even spherical $\mathrm{Pb}$ and Sn nuclei $[5,30,98]$ and do not modify their charge radii substantially $[5,30]$. Thus, the treatment of the ground states of such nuclei at the mean-field level represents a reasonable approximation. In this approximation the physical observables of interest are defined by the single-particle properties and the occupation probabilities $\mathrm{v}_{\text {state }}^{2}(N)$ of these states (see left and right columns of Fig. 30).

In contrast, PVC in odd- $A$ nuclei leads to a substantial fragmentation of many single-particle states (including the ground state) which is experimentally observed (see Refs. [66-68, 99-103] and references quote therein). As schematically shown in the middle part of Fig. 30, each mean-field state $k$ ( $k=i$ or $j$ in this figure) with energy $\varepsilon_{k}$ is split into many levels due to PVC, so the single-particle strength is fragmented over many levels. In the diagonal approximation for the nucleonic selfenergy, these levels have the same quantum numbers as the original mean-field state $k$, but different energies $\varepsilon_{k}^{\nu}$ and spectroscopic factors $S_{k}^{\nu}$. In the PVC model, the spectroscopic factors, which are the real numbers between zero and one, play a role of the occupation probabilities of these fragmented states satisfying the sum rule $\sum_{\nu} S_{k}^{\nu}=1$. For the states in the vicinity of the Fermi surface, one dominant level with $0.5 \leq S_{k}^{\nu} \leq 1.0$ and many other levels with small $S_{k}^{\nu}$ are usually obtained. Both in experiment and in the calculations, the dominant singleparticle state is typically the lowest in energy among the set of fragmented states originating from the mean-field state $k$. However, for the mean-field states located far away from the Fermi surface, one observes a very strong splitting over many levels with much smaller and comparable spectroscopic factors.

The detailed global analysis of the impact of the occupation of neutron single-particle orbitals in the vicinity of spherical neutron shell closures presented in Fig. 31 reveals a strong correlation between the principal quantum number $n$ of the single neutron orbital occupied above the neutron shell closure and the impact of the occupation of this orbital on differential charge radii. One can see that in a given isotopic chain the largest impact on $\delta\left\langle r^{2}\right\rangle^{N, N^{\prime}}$ is provided by the occupation of the orbital with the lowest $n$. This feature has already been revealed for the $\mathrm{Pb}$ isotopes in Ref. [6] but the present study generalizes it to a larger set of the nuclei and exposes new features. For example, it uncovers that for the $n=1$ orbitals this feature is strictly speaking true only for the occupation of the orbitals located above the shell closure the spin-orbit partner orbitals of which are fully occupied below this shell closure. Indeed, this is the case for the " $j-1 / 2 "$ type $1 i_{11 / 2}, 1 h_{9 / 2}, 1 g_{7 / 2}$ and $1 f_{5 / 2}$ orbitals the " $j+1 / 2$ " spin-orbit partners of which, namely, $1 i_{13 / 2}, 1 h_{11 / 2}, 1 g_{9 / 2}$ and $1 f_{7 / 2}$, are fully occupied below the $N^{\prime}=126,82,50$ and 28 neutron shell closures in the $\mathrm{Pb}, \mathrm{Sn}, \mathrm{Sr}$ and $\mathrm{Ca}$ 


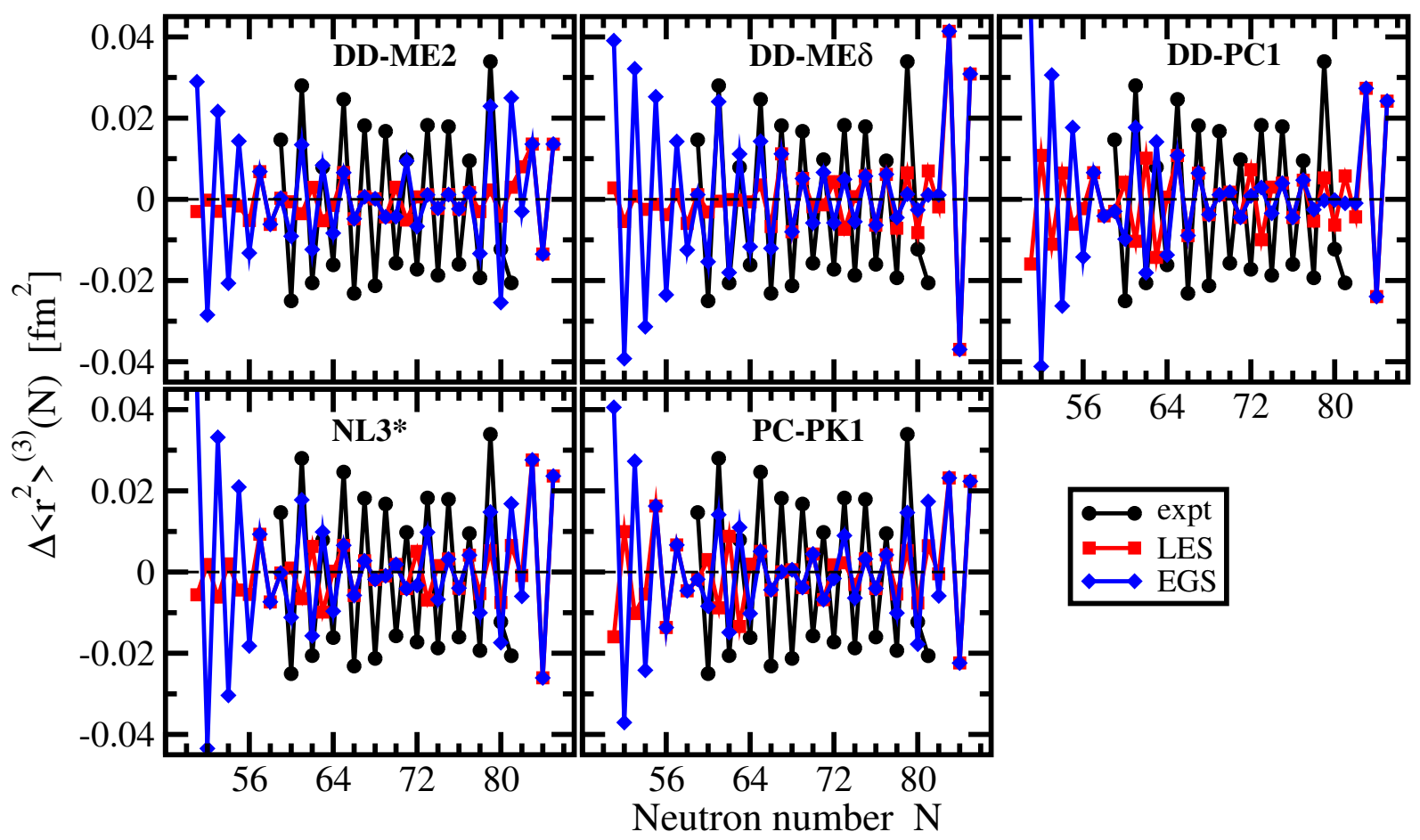

FIG. 29. The OES in charge radii of the Sn isotopes. The experimental data are taken from Ref. [2].

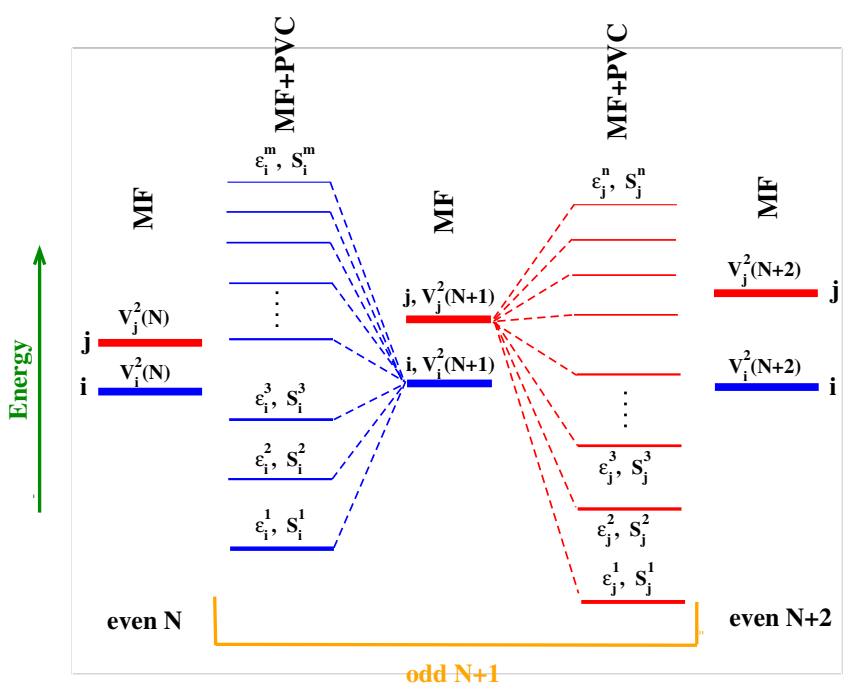

FIG. 30. Schematic illustration of the difference of the approximations ( $\mathrm{MF}$ and $\mathrm{MF}+\mathrm{PVC}$ ) used in the description of even-even and odd mass nuclei (see text for details). "MF" and "MF+PVC" stands for "mean-field" and "mean-field + particle vibration coupling" approximations, respectively. The thickness of the horizontal lines is proportional to spectroscopic factors $S_{k}^{m}$ in the $\mathrm{MF}+\mathrm{PVC}$ columns.

nuclei under study. However, the differential charge radius in the $\mathrm{Pb}$ isotopes provided by the occupation of the $n=11 j_{15 / 2}$ orbital located above the $N^{\prime}=126$ shell closure (see Fig. 1(b)) is only approximately half of that provided by the $1 i_{11 / 2}$ one and it is not far away from differential charge radii generated by occupation of the $n=2$ orbitals (see Fig. 31). In addition, Fig. 31 shows that there are no clear correlations between $\delta\left\langle r^{2}\right\rangle^{N, N^{\prime}}$ and $r_{\text {neu }}^{s p}$. It is also interesting that the differential charge radii for a given $n$ only weakly depend both on the mass of the nucleus and $r_{n e u}^{s p}$. In addition, within the spinorbit doublet the occupation of the lower lying partner orbital with $j+1 / 2$ provides smaller differential charge radii than the occupation of the higher lying partner orbital with $j-1 / 2$ (see Fig. 31). This is because the latter ones have large neutron radii as compared with former ones.

As a consequence, the single-particle content of unpaired neutron states in odd- $A$ nuclei plays an important role in our understanding of OES in charge radii since it defines the pull on charge densities (see also the discussion in the introduction). One of the ways to modify this content is via the pairing interaction (see Sec. IX B). Another is via the fragmentation of the single-particle states by means of PVC. Indeed it reduces substantially (down to $60-90 \%[67,68,102])$ their full single-particle content and this fact is experimentally confirmed. A strict way to calculate the OES effect in charge radii in the presence of beyond mean field effects would be to perform quasiparticle random phase approximation (QRPA) calculations in even-even $(Z, N)$ nucleus and then PVC calculations in odd- $A(Z, N+1)$ nucleus and then to define the differential charge radius. Note that the PVC calculations in the latter nucleus use the $(Z, N)$ core with QRPA correlations included and then adds particle-vibration coupling $[67,68,102]$. Existing calculations show that away 


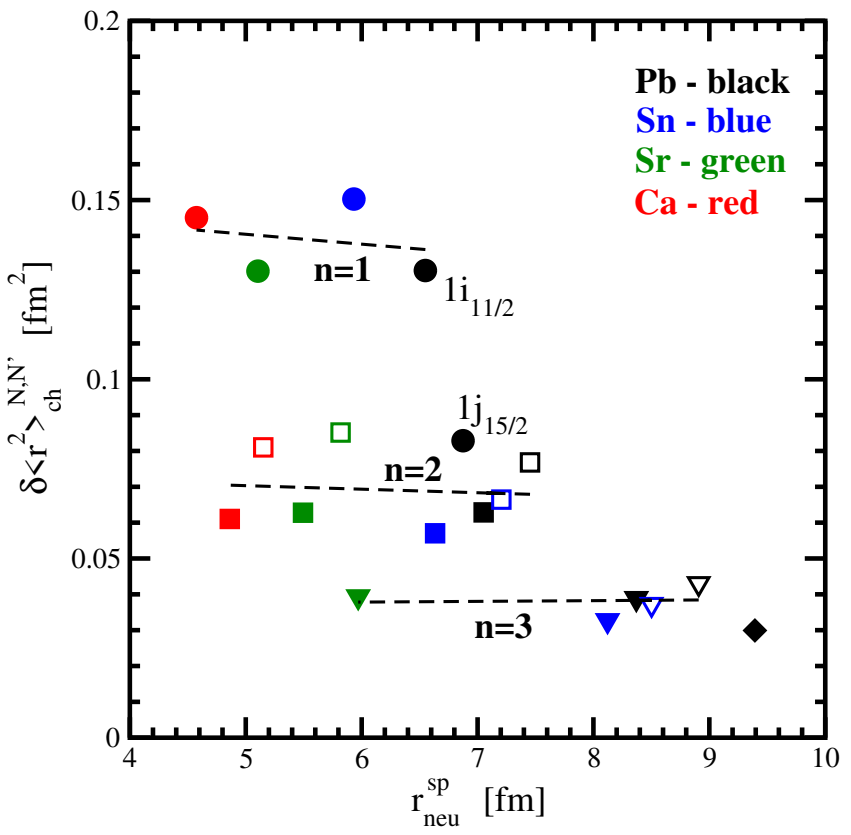

FIG. 31. The correlations between the differential charge radii $\delta\left\langle r^{2}\right\rangle^{N, N^{\prime}}\left(N=N^{\prime}+1\right)$ generated by the occupation of a given single neutron orbital above the neutron shell closure located at $N^{\prime}$ and the rms neutron radius $r_{n e u}^{s p}=\sqrt{\left\langle r^{2}\right\rangle^{s p}}$ of this orbital. The results presented for the ${ }^{48,49} \mathrm{Ca}\left(N^{\prime}=\right.$ $28),{ }^{88,89} \mathrm{Sr}\left(N^{\prime}=50\right),{ }^{132,133} \mathrm{Sn}\left(N^{\prime}=82\right)$ and ${ }^{208,209} \mathrm{~Pb}$ $\left(N^{\prime}=126\right)$ are based on the calculations without pairing and the NL3* CEDF. The calculations are restricted to spherical shapes. Circles, squares, triangles and diamonds are used for the orbitals with principal quantum numbers $n=1,2,3$ and 4 , respectively. If spin-orbit partner orbitals appear above the shell closure, then solid (open) symbols of the same type are used for the $j+1 / 2(j-1 / 2)$ ones. The following neutron orbitals are considered: $1 i_{11 / 2}, 1 j_{15 / 2}, 2 g_{9 / 2}, 2 g_{7 / 2}, 3 d_{5 / 2}$, $3 d_{3 / 2}$ and $4 s_{1 / 2}$ in ${ }^{208,209} \mathrm{~Pb}$ (see Fig. $1(\mathrm{~b})$ ), $1 h_{9 / 2}, 2 f_{7 / 2}$, $2 f_{5 / 2}, 3 p_{3 / 2}$ and $3 p_{1 / 2}$ in ${ }^{132,133} \mathrm{Sn}$ (see Fig. $14(\mathrm{~b})$ ), $1 g_{7 / 2}$, $2 d_{5 / 2}, 2 d_{3 / 2}$ and $3 s_{1 / 2}$ in ${ }^{88,89} \mathrm{Sr}$ and $1 f_{5 / 2}, 2 p_{3 / 2}$ and $2 p_{1 / 2}$ in ${ }^{48,49} \mathrm{Ca}$ (see Fig. 21(b)). Dashed lines show average trends for different values of $n$.

from the vicinity of doubly magic shell closures eveneven cores supplemented by QRPA correlations behave smoothly as a function of neutron number (see Ref. [30]) and their contributions to charge radii are rather modest. Because of these reasons, the addition of the neutron is not expected to modify the proton part of the core in the PVC calculations. The detailed investigation of OES in differential charge radii requires a fully fledged PVC calculations which will be undertaken in a future.

At this point, we want to estimate whether the depletion of the single-particle content of the wave function in odd- $N$ nuclei due to fragmentation could lead to a right phase (defined as a the sign of $\Delta\left\langle r^{2}\right\rangle^{(3)}(N)$ at given $N)$ and magnitude of OES in charge radii. The basic assumptions behind the discussion below are the following. First, we use the fact that beyond mean field ground

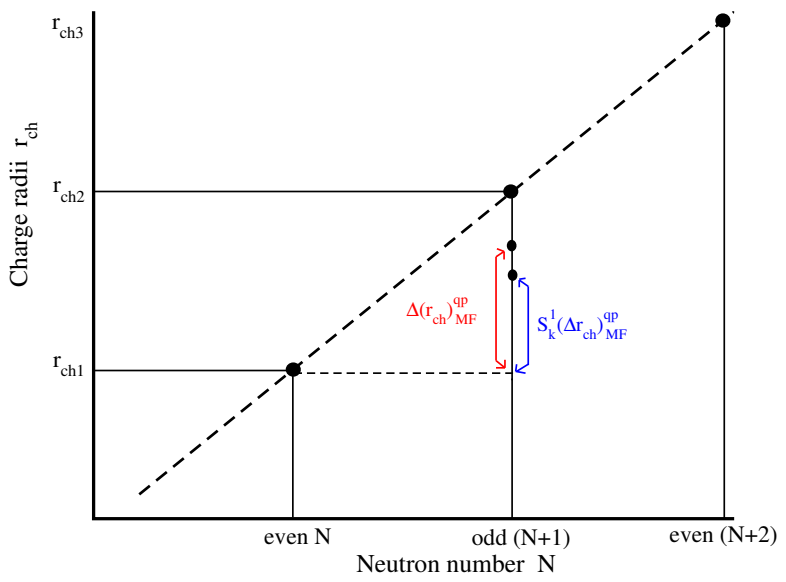

FIG. 32. Schematic illustration of the impact of pairing and particle-vibration coupling on charge radii in odd- $N$ nuclei. The dashed straight line corresponds to a linear increase of charge radii due to a sequential occupation of the single-particle subshell in the calculations without pairing. $\Delta\left(r_{c h}\right)_{M F}^{q p}$ corresponds to an increase of the charge radii in odd- $N$ as compared with the one given in the system with even- $N$ neutrons when one neutron is added in the calculations with pairing. $S_{k}^{1} \Delta\left(r_{c h}\right)_{M F}^{q p}$ provides a similar quantity for the case when the depletion of the single-particle content due to fragmentation is additionally taken into account (see text for details).

state correlations in even-even spherical nuclei and their impact on charge radii are rather small and that they behave smoothly as a function of neutron number (see Refs. $[5,30,98])$. Second, the wavefunction of the odd-neutron ground state in the odd- $N$ nucleus represents a superposition of single-particle and vibrational contributions. The pull on proton densities is provided predominantly by the former while the latter is not expected to provide a significant contribution to the differential charge radii. This is because these vibrational contributions (i) are the superposition of two-quasiparticle states the wavefunctions of which have only a small overlap with that of the ground states of even-even cores and (ii) even-even cores are very similar in neighbouring even-even and odd- $A$ nuclei.

In this kind of situation it is reasonable to expect that (i) the average behavior of charge radii as a function of neutron number can be reasonably well approximated by the mean field [since anyway it does not provide a contribution to OES of charge radii] and (ii) only the part of the single neutron in the odd- $N$ nucleus defined by the spectroscopic factor $S_{k}^{1}$ of the dominant single-particle level provides a pull on proton densities and thus the leading contribution to the oscillating part of $\Delta\left\langle r^{2}\right\rangle^{(3)}(N)$. The latter leads to a reduction of the increase of charge radii in going from the even- $N$ to the odd- $N$ nucleus from $\Delta\left(r_{c h}\right)_{M F}^{q p}$ at the mean-field level to approximately $S_{k}^{1} \Delta\left(r_{c h}\right)_{M F}^{q p}$ when the fragmentation of the dominant single-particle level is taken into account (see Fig. 30).

The impact of this modification for representative val- 
ues of the spectroscopic factors $S_{k}^{1}=0.9,0.8$ and 0.7 is illustrated in Fig. 33. One can see that additional fragmentation of the structure of the unpaired neutron in odd- $N$ nuclei leads to an increase of the magnitudes of OES in charge radii and correct phases of the OES both in the LES and EGS procedures. With the spectroscopic factors $S_{k}^{1}$ being in the vicinity of those calculated in Refs. $[67,68,98,102]$, the calculated OES are close to experimental ones in the Sn isotopes (see Fig. 33).

Note that in some cases, PVC leads to a change of the relative order of the single-particle states obtained at the mean-field level. Such a possibility is illustrated in Fig. 30 . In the odd- $N$ nucleus, the state $i$ is lower in energy than the $j$ state at the MF level, and both states have single-particle nature. In contrast, in the $\mathrm{MF}+\mathrm{PVC}$ case, the fragmented level with a dominant single-particle $j$ state component and the energy $\varepsilon_{j}^{1}$ is lower in energy than the fragmented level with the dominant single-particle component $i$ and the energy $\varepsilon_{i}^{1}$. This feature has been used in Ref. [17] for a simultaneous explanation of the kink in charge radii at $N=126$ and the OES in charge radii of the $\mathrm{Pb}$ and $\mathrm{Hg}$ isotopes with $N>126$.

\section{Other sources of the inversion of OES}

The inverted OES is also observed in the At (for $N=133), \operatorname{Rn}$ (for $N=133-135), \operatorname{Fr}$ (for $N=135-138$ ), Ra (for $N=133-138$ and 140), and Ac (for $N=137$ ) isotopic chains [see Fig. 26(t) and (u) and Figs. 27(o), (p) and $(\mathrm{s})]$. It is frequently attributed to the effect of octupole deformation (see Refs. [19, 20, 69]. For example, it was suggested in Refs. $[104,105]$ that the OES inversion originates from the fact that octupole deformation should be more pronounced in odd than in even nuclei. This leads to a charge radius of the odd- $N$ nucleus being larger than the average charge radius of the two eveneven neighbors. However, non-relativistic Skyrme DFT calculations for the Ac isotopes presented in Ref. [20] show that this is not necessary the case since such an inversion appears at some neutron numbers even in the calculations without octupole deformation. Theoretical models also differ in the prediction of static octupole deformation in the Rn isotopes (see Table I in Ref. [106] and Ref. [107]). However, experimental data presented in Refs. [108, 109] strongly suggests that ${ }^{218-222} \mathrm{Rn} n u-$ clei behave like octupole vibrators and not like the nuclei with static octupole deformation. We also have to keep in mind that the picture of a static octupole deformation and the coupling to dynamic octupole vibrations has much in common. Both models describe in many ways, but not completely, the same physics of static and dynamic polarization effects (see, for instance, Ref. [110], where the same problem has been discussed in detail for static pairing correlations and pairing vibrations).

In addition, there are other isotopic chains in which the OES is inverted either locally (for a few neutron numbers only) or over a substantial range of neutron numbers.
These are isotopic chains of $\mathrm{Kr}, \mathrm{Sr}, \mathrm{Rb}, \mathrm{Ba}, \mathrm{Sm}, \mathrm{Cs}$, $\mathrm{Yb}, \mathrm{Hf}, \mathrm{Eu}, \mathrm{Lu}$, and Ir [see Figs.26(e), (f), (j), (l), (n), and (o) and Figs. 27(c), (e), (f), (j) and (k)]. The octupole deformation is not present in the ground states of these nuclei, so there should be other sources of the OES inversion different from octupole deformation. For example, it was speculated in Ref. [111] that OES in charge radii of light $\mathrm{Kr}, \mathrm{Rb}$, and $\mathrm{Sr}$ nuclei is due to a polarization effect of the even-even core by the unpaired neutron, driving the odd- $N$ nuclei toward stronger quadrupole deformation [as compared with the average given by even$N$ neighbors]. However, this was not supported by any model calculation.

Note that all above discussed cases involve deformed nuclei, and these isotopic chains include both odd and odd-odd nuclei. Because of the complexity of the description of such nuclei (see Refs. [64, 112]) a detailed investigation of OES and its inversion in these isotopic chains goes beyond the scope of the present paper, but it is planned for the future.

\section{CONCLUSIONS}

A systematic global investigation of differential charge radii has been performed within the CDFT framework for the first time. Theoretical results obtained with conventional covariant energy density functionals and the separable pairing interaction of Ref. [1] are compared with experimental differential charge radii in the regions of the nuclear chart where available experimental data crosses neutron shell closures at $N=28,50,82$, and 126 . The main results can be summarized as follows:

- In spherical nuclei, the appearance of the kinks in the $\delta\left\langle r^{2}\right\rangle^{N, N^{\prime}}$ curves at neutron shell closures is defined predominantly in the particle-hole channel of the CDFT with details of the single-particle structure above shell closures playing an important role. This conclusion is different from the one obtained in nonrelativistic Skyrme and Fayans DFTs in Ref. [15] which indicates that pairing is the dominant contributor to the kink. In the RHB approach, the kinks are already present in the calculations without pairing. Pairing acts only as an additional tool that mixes different configurations and somewhat softens the evolution of charge radii as a function of neutron number.

- The relative energies of the single-particle states and the patterns of their occupation with increasing neutron number have a significant impact on the evolution of the $\delta\left\langle r^{2}\right\rangle^{N, N^{\prime}}$ values even in the calculations with pairing included. Considering existing inaccuracies in the description of the energies of the single-particle states in the DFT calculations, the predictive power of such models for $\delta\left\langle r^{2}\right\rangle^{N, N^{\prime}}$ 

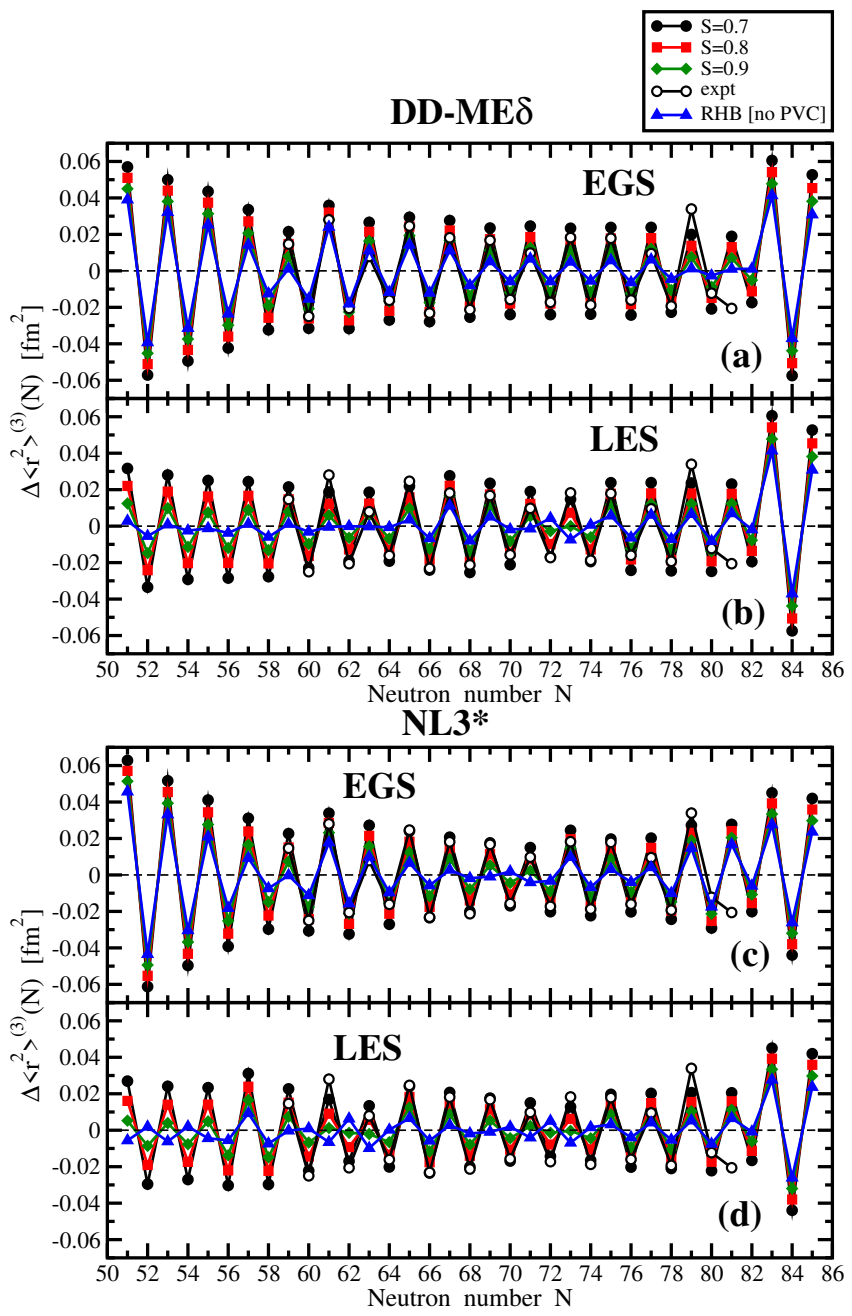

FIG. 33. OES in charge radii of the Sn isotopes corrected for the fragmentation of the single-particle content of the dominant single-particle state in odd- $N$ nuclei by PVC coupling within the framework of a schematic model discussed in the text for different values of the spectroscopic factor $S=S_{k}^{1}$.

is expected to decrease in the regions of high densities of the single-particle states of different origin.

- The analysis of absolute differential radii of different isotopic chains and their relative properties clearly indicate that such properties are reasonably well described in model calculations in cases where the mean-field approximation is justified. The analysis of potential energy curves provides the latter justification. However, it turns out that it is more difficult to describe the clusterization of the differential charge radii in the $\mathrm{Sn}$ and $\mathrm{Ca}$ regions for neutron numbers above shell closures at $N=82$ and 28 since it depends on the details of the underlying single-particle structure.

- There are regions of the nuclear chart where the description at the mean-field level faces difficulties in reproducing experimental data. In the CDFT calculations, these are the Ca isotopes, the $N<50$ and $N>58$ nuclei in the $\mathrm{Sr}$ region and the neutronpoor nuclei in the $\mathrm{Pb}$ region. The latter two regions are characterized by shape coexistence, and, in many cases, the assignment of the calculated excited prolate minimum to the experimental groundstate allows understanding the trends of the evolution of differential charge radii with neutron number. The inclusion of beyond mean-field effects could possibly improve the description of charge radii is these systems. As follows from the comparison of the calculated and experimental masses in Ref. [4] and from increased [as compared with heavy nuclei] OES in charge radii of very light nuclei [see Figs. 26(a), (b) and (c) and Figs. 27(a)], such effects are expected to play a significant role in the properties of the ground states of light nuclei. That was a reason why light nuclei have been excluded from the analysis in the present paper.

- It is usually assumed that pairing is the dominant contributor to OES in charge radii. Our analysis paints a more complicated picture and suggests a new additional mechanism where the fragmentation of the single-particle content of the ground state in odd-mass nuclei due to particle-vibration coupling provides a significant contribution to OES in charge radii. Note that similarly, the pairing indicators, which depend on OES of binding energies, are also expected to be affected by particle-vibration coupling with its impact to be especially pronounced in spherical nuclei (see Ref. [113]).

- The PECs curves obtained in the calculations with the CEDF DD-ME $\delta^{13}$ quite frequently deviate from those obtained with DD-ME2, DD-PC1, NL3* and PC-PK1. This could also affect the results of beyond mean field calculations making them in some nuclei significantly dissimilar for DD-ME $\delta$ as compared with above mentioned functionals.

This difference could be due to two factors, making DD-ME $\delta$ substantially different from the other conventional CDFTs:

First, DD-ME $\delta$ has less fit parameters, and therefore the adjustment of this CEDF could be less successful: DD-ME $\delta$ is the most microscopic CEDF. Only four parameters at the saturation density are fitted to finite nuclei and the full density dependence of the parameters is derived from ab-initio

13 Note that the DD-ME $\delta$ CEDF provides a reasonable global description of the ground state properties (see Ref. [4]), but it fails to predict octupole deformed actinides [106] and generates fission barriers in superheavy nuclei which are too small to make them relatively stable [114]. Thus, it is not recommended for applications to nuclei heavier than lead. 
calculations [47]. On the contrary, the other interactions contain an additional 2 (NL3*), 4 (DDME2), 5 (PC-PK1), and 6 (DD-PC1) phenomenological parameters for the fine-tuning of different channels of CEDFs and their density dependence. Note that not all of these additional parameters are independent (see Refs. [75, 78]).

Second, in addition to the three spin-isospin channels represented by the $\sigma-, \omega$-, and $\rho$-meson, DD$\mathrm{ME} \delta$ also contains, as the only parameter set considered here, an isovector scalar channel represented by the $\delta$-meson. This influences the isospin dependence of the spin-orbit field and, therefore, that of the single-particle energies. However, it is practically impossible to adjust the parameters of the $\delta$-meson to experimental data because (i) there is very little data on the isospin dependence of single-particle energies and the largely unknown influence of tensor forces and of particle vibrational coupling [68] forbids the fitting to single-particle levels anyhow, and (ii) it has been shown in Ref. [47], that the parameters of the $\delta$-meson cannot be determined by fitting to the usual bulk properties of finite nuclei, because here the changes in the parameters of the $\delta$-meson are completely com- pensated by corresponding changes in the remaining isovector channel, i.e. by the $\rho$-meson [47]. Therefore for the CEDF DD-ME $\delta$, in Ref. [47], the strength and the density dependence of the $\delta$ nucleon vertex have been adjusted to ab-initio results, i.e. to the isovector effective mass $m_{p}^{*}-m_{n}^{*}$, derived from relativistic Brueckner theory in Ref. [115]. These relativistic Brueckner-Hartree-Fock (RBHF) calculations suffer from the fact that the Thompson equation has not been treated in full Dirac space, and the coupling to negative energy solutions is only treated approximately. Only recently the RBHF calculations for symmetric nuclear matter have been carried out in full Dirac space [116], but corresponding solutions for asymmetric nuclear matter are still missing.

\section{ACKNOWLEDGMENTS}

This material is based upon work supported by the U.S. Department of Energy, Office of Science, Office of Nuclear Physics under Award No. DE-SC0013037. PR acknowledges partial support from the Deutsche Forschungsgemeinschaft (DFG, German Research Foundation) under under Germany Excellence Strategy EXC2094-390783311, ORIGINS.
[1] Y. Tian, Z. Y. Ma, and P. Ring, A finite range pairing force for density functional theory in superfluid nuclei, Phys. Lett. B 676, 44 (2009).

[2] I. Angeli and K. P. Marinova, Table of experimental nuclear ground state charge radii: An update, At. Data Nucl. Data Tables 99, 69 (2013).

[3] P. Campbell, I. Moore, and M. Pearson, Laser spectroscopy for nuclear structure physics, Prog. Part. Nucl. Phys. 86, 127 (2016).

[4] S. E. Agbemava, A. V. Afanasjev, D. Ray, and P. Ring, Global performance of covariant energy density functionals: Ground state observables of even-even nuclei and the estimate of theoretical uncertainties, Phys. Rev. C 89, 054320 (2014).

[5] P.-G. Reinhard and H. Flocard, Nuclear effective forces and isotope shifts, Nucl. Phys. A 584, 467 (1995).

[6] P. M. Goddard, P. D. Stevensson, and A. Rios, Charge radius isotope shift across the $n=126$ shell gap, Phys. Rev. Lett. 110, 032503 (2013).

[7] A. V. Afanasjev and H. Abusara, From cluster structures to nuclear molecules: The role of nodal structure of the single-particle wave functions, Phys. Rev. C 97, 024329 (2018).

[8] A. V. Afanasjev, Cluster structures, ellipsoidal shapes and nuclear molecules in light $a=12-50$ nuclei, Eur. Phys. J., Web of Conf. 194, 06001 (2018).

[9] S. G. Nilsson and I. Ragnarsson, Shapes and shells in nuclear structure, Shapes and shells in nuclear structure, (Cambridge University Press, 1995).
[10] A. Koszorús, X. F. Yang, W. G. Jiang, S. J. Novario, S. W. Bai, J. Billowes, C. L. Binnersley, M. L. Bissell, T. E. Cocolios, B. S. Cooper, R. P. de Groote, A. Ekström, K. T. Flanagan, C. Forssén, S. Franchoo, R. F. Garcia-Ruiz, F. P. Gustafsson, G. Hagen, G. R. Jansen, A. Kanellakopoulos, M. Kortelainen, W. Nazarewicz, G. Neyens, T. Papenbrock, P.-G. Reinhard, C. M. Ricketts, B. K. Sahoo, A. R. Vernon, and S. G. Wilkins, Charge radii of exotic potassium isotopes challenge nuclear theory and the magic character of $n=32$, Nature Physics 17, 539 (2021).

[11] R. F. Garcia-Ruiz, M. L. Bissell, K. Blaum, A. Schwenk, J. Simonis, K. A. Wendt, and D. T. Yordanov, Unexpectedly large charge radii of neutron-rich calcium isotopes, Nature Physics 12, 594 (2016).

[12] A. J. Miller, K. Minamisono, A. Klose, D. Garand, C. Kujawa, J. D. Lantis, Y. Liu, B. Maa, P. F. Mantica, W. Nazarewicz, W. Nörtershäuser, S. V. Pineda, P.-G. Reinhard, D. M. Rossi, F. Sommer, C. Sumithrarachchi, A. Teigelhöfer, and J. Watkins, Proton superfluidity and charge radii in proton-rich calcium isotopes, Nat. Phys. 15, 432 (2019).

[13] R. P. de Groote, J. Billowes, C. L. Binnersley, M. L. Bissell, T. E. Cocolios, T. D. Goodacre, G. J. FarooqSmith, D. V. Fedorov, K. T. Flanagan, S. Franchoo, R. F. Garcia-Ruiz, W. Gins, J. D. Holt, . Koszorús, K. M. Lynch, T. Miyagi, W. Nazarewicz, G. Neyens, P.-G. Reinhard, S. Rothe, H. H. Stroke, A. R. Vernon, K. D. A. Wendt, S. G. Wilkins, Z. Y. Xu, and X. F. Yang, Measurement and microscopic description of odd- 
even staggering of charge radii of exotic copper isotopes, Nature Phys. 16, 620 (2020).

[14] M. Hammen, W. Nörtershäuser, D. L. Balabanski, M. L. Bissell, K. Blaum, I. Budinčević, B. Cheal, K. T. Flanagan, N. Frömmgen, G. Georgiev, C. Geppert, M. Kowalska, K. Kreim, A. Krieger, W. Nazarewicz, R. Neugart, G. Neyens, J. Papuga, P.-G. Reinhard, M. M. Rajabali, S. Schmidt, and D. T. Yordanov, From calcium to cadmium: Testing the pairing functional through charge radii measurements of ${ }^{100-130} \mathrm{Cd}$, Phys. Rev. Lett. 121, 102501 (2018).

[15] C. Gorges, L. V. Rodríguez, D. L. Balabanski, M. L. Bissell, K. Blaum, B. Cheal, R. F. Garcia Ruiz, G. Georgiev, W. Gins, H. Heylen, A. Kanellakopoulos, S. Kaufmann, M. Kowalska, V. Lagaki, S. Lechner, B. Maaß, S. Malbrunot-Ettenauer, W. Nazarewicz, R. Neugart, G. Neyens, W. Nörtershäuser, P.-G. Reinhard, S. Sailer, R. Sánchez, S. Schmidt, L. Wehner, C. Wraith, L. Xie, Z. Y. Xu, X. F. Yang, and D. T. Yordanov, Laser spectroscopy of neutron-rich tin isotopes: A discontinuity in charge radii across the $n=82$ shell closure, Phys. Rev. Lett. 122, 192502 (2019).

[16] D. T. Yordanov, L. V. Rodríguez, D. L. Balabanski, J. Bieroń, M. L. Bissell, K. Blaum, B. Cheal, J. Ekman, G. Gaigalas, R. F. Garcia-Ruiz, G. Georgiev, W. Gins, M. R. Godefroid, C. Gorges, Z. Harman, H. Heylen, P. Jönsson, A. Kanellakopoulos, S. Kaufmann, C. H. Keitel, V. Lagaki, S. Lechner, B. Maa, S. Malbrunot-Ettenauer, W. Nazarewicz, R. Neugart, G. Neyens, W. Nörtershäuser, N. S. Oreshkina, A. Papoulia, P. Pyykkö, P.-G. Reinhard, S. Sailer, R.Sánchez, S. Schiffmann, S. Schmidt, L. Wehner, C. Wraith, L. X. Z. Xu, and X. Yang, Structural trends in atomic nuclei from laser spectroscopy of tin, Comm. Phys. 3, 107 (2020).

[17] T. D. Goodacre, A. V. Afanasjev, A. E. Barzakh, B. A. Marsh, S. Sels, P. Ring, H. Nakada, A. N. Andreyev, P. V. Duppen, N. A. Althubiti, B. Andel, D. Atanasov, J. Billowes, K. Blaum, T. E. Cocolios, J. G. Cubiss, G. J. Farooq-Smith, D. V. Fedorov, V. N. Fedosseev, K. T. Flanagan, L. P. Gaffney, L. Ghys, M. Huyse, S. Kreim, D. Lunney, K. M. Lynch, V. Manea, Y. M. Palenzuela, P. L. Molkanov, M. Rosenbusch, R. E. Rossel, S. Rothe, L. Schweikhard, M. D. Seliverstov, P. Spagnoletti, C. V. Beveren, M. Veinhard, E. Verstraelen, A. Welker, K. Wendt, F. Wienholtz, R. N. Wolf, A. Zadvornaya, and K. Zuber, Laser spectroscopy of neutron-rich ${ }^{207,208} \mathrm{hg}$ isotopes: Illuminating the kink and odd-even staggering in charge radii across the $\mathrm{n}=$ 126 shell closure, Phys. Rev. Let. 126, 032502 (2021).

[18] A. E. Barzakh, D. V. Fedorov, V. S. Ivanov, P. L. Molkanov, F. V. Moroz, S. Y. Orlov, V. N. Panteleev, M. D. Seliverstov, and Y. M. Volkov, Shell effect in the mean square charge radii and magnetic moments of bismuth isotopes near $n=126$, Phys. Rev. C 97, 014322 (2018).

[19] A. E. Barzakh, J. G. Cubiss, A. N. Andreyev, M. D. Seliverstov, B. Andel, S. Antalic, P. Ascher, D. Atanasov, D. Beck, J. Bieron, K. Blaum, C. Borgmann, M. Breitenfeldt, L. Capponi, T. E. Cocolios, T. Day Goodacre, X. Derkx, H. De Witte, J. Elseviers, D. V. Fedorov, V. N. Fedosseev, S. Fritzsche, L. P. Gaffney, S. George, L. Ghys, F. P. Heßberger, M. Huyse, N. Imai, Z. Kalaninová, D. Kisler, U. Köster, M. Kowalska, S. Kreim, J. F. W. Lane, V. Liberati, D. Lunney, K. M. Lynch,
V. Manea, B. A. Marsh, S. Mitsuoka, P. L. Molkanov, Y. Nagame, D. Neidherr, K. Nishio, S. Ota, D. Pauwels, L. Popescu, D. Radulov, E. Rapisarda, J. P. Revill, M. Rosenbusch, R. E. Rossel, S. Rothe, K. Sandhu, L. Schweikhard, S. Sels, V. L. Truesdale, C. Van Beveren, P. Van den Bergh, P. Van Duppen, Y. Wakabayashi, K. D. A. Wendt, F. Wienholtz, B. W. Whitmore, G. L. Wilson, R. N. Wolf, and K. Zuber, Inverse odd-even staggering in nuclear charge radii and possible octupole collectivity in ${ }^{217,218,219}$ At revealed by in-source laser spectroscopy, Phys. Rev. C 99, 054317 (2019).

[20] E. Verstraelen, A. Teigelhöfer, W. Ryssens, F. Ames, A. Barzakh, M. Bender, R. Ferrer, S. Goriely, P.-H. Heenen, M. Huyse, P. Kunz, J. Lassen, V. Manea, S. Raeder, and P. Van Duppen, Search for octupoledeformed actinium isotopes using resonance ionization spectroscopy, Phys. Rev. C 100, 044321 (2019).

[21] S. Raeder, D. Ackermann, H. Backe, R. Beerwerth, J. C. Berengut, M. Block, A. Borschevsky, B. Cheal, P. Chhetri, C. E. Düllmann, V. A. Dzuba, E. Eliav, J. Even, R. Ferrer, V. V. Flambaum, S. Fritzsche, F. Giacoppo, S. Götz, F. P. Heßberger, M. Huyse, U. Kaldor, O. Kaleja, J. Khuyagbaatar, P. Kunz, M. Laatiaoui, F. Lautenschläger, W. Lauth, A. K. Mistry, E. Minaya Ramirez, W. Nazarewicz, S. G. Porsev, M. S. Safronova, U. I. Safronova, B. Schuetrumpf, P. Van Duppen, T. Walther, C. Wraith, and A. Yakushev, Probing sizes and shapes of nobelium isotopes by laser spectroscopy, Phys. Rev. Lett. 120, 232503 (2018).

[22] S. O. Allehabi, V. A. Dzuba, V. V. Flambaum, A. V. Afanasjev, and S. E. Agbemava, Using isotope shift for testing nuclear theory: The case of nobelium isotopes, Phys. Rev. C 102, 024326 (2020).

[23] R. F. G. Ruiz and A. Vernon, Emergence of simple patterns in many-body systems: from macrosocpic objects to the atomic nucleus, Eur. Phys. J. A 56, 136 (2020).

[24] N. Tajima, P. Bonche, H. Flocard, P.-H. Heenen, and M. S. Weiss, Self-consistent calculation of charge radii of pb isotopes, Nucl. Phys. A 551, 434 (1993).

[25] M. M. Sharma, G. A. Lalazissis, and P. Ring, Anomaly in the charge radii of pb isotopes, Phys. Lett. B 317, 9 (1993).

[26] M. M. Sharma, G. Lalazissis, J. König, and P. Ring, Isospin dependence of the spin-orbit force and effective nuclear potentials, Phys. Rev. Lett. 74, 3744 (1995).

[27] H. Nakada, Further evidence for three-nucleon spinorbit interaction in isotope shifts of nuclei with magic proton numbers, Phys. Rev. C 92, 044307 (2015).

[28] S. A. Fayans, S. V. Tolokonnikov, E. L. Trykov, and D. Zawischa, Isotope shifts within the energy-density functional approach with density dependent pairing, Phys. Lett. B 338, 1994 (1).

[29] S. A. Fayans and D. Zawischa, Towards a better parametrization of the nuclear pairing force: density dependence with gradient term, Phys. Lett. B 383, 19 (1996).

[30] S. A. Fayans, S. V. Tolokonnikov, E. L. Trykov, and D. Zawischa, Nuclear isotope shifts within the local energy-density functional approach, Nucl. Phys. A 676, 49 (2000).

[31] P.-G. Reinhard and W. Nazarewicz, Toward a global description of nuclear charge radii: Exploring the fayans energy density functional, Phys. Rev. C 95, 064328 
(2017).

[32] R. Rodríguez-Guzmán, P. Sarriguren, L. M. Robledo, and S. Perez-Martin, Charge radii and structural evolution in sr, zr, and mo isotopes, Phys. Lett. B 691, 202 (2010).

[33] H. Nakada, Properties of exotic nuclei and their linkage to the nucleonic interaction, J. Phys. G 46, 013001 (2019).

[34] H. Nakada and T. Inakura, Effects of three-nucleon spinorbit interaction on isotope shifts of pb nuclei, Phys. Rev. C 91, 021302(R) (2015).

[35] H. Nakada, Irregularities in nuclear radii at magic numbers, Phys. Rev. C 100, 044310 (2019).

[36] A. V. Afanasjev and S. E. Agbemava, Covariant energy density functionals: Nuclear matter constraints and global ground state properties, Phys. Rev. C 93, 054310 (2016).

[37] Mass Explorer: DFT mass tables at http://massexplorer.frib.msu.edu/content/DFTMassTa

[38] R. Utama, W.-C. Chen, and J. Piekarewicz, Nuclear charge radii: density functional theory meets bayesian neural networks, J. Phys. G 43, 114002 (2016).

[39] E. Yüksel, T. Marketin, and N. Paar, Optimizing the relativistic energy density functional with nuclear ground state and collective excitation properties, Phys. Rev. C 99, 034318 (2019).

[40] R. An, L. Geng, and S.-S. Zhang, Novel ansatz for charge radii in density functional theories, Phys. Rev. C 102, 024307 (2020).

[41] A. V. Afanasjev and O. Abdurazakov, Pairing and rotational properties of actinides and superheavy nuclei in covariant density functional theory, Phys. Rev. C 88, 014320 (2013).

[42] Z.-H. Zhang, M. Huang, and A. V. Afanasjev, Rotational excitations in rare-earth nuclei: A comparative study within three cranking models with different mean fields and treatments of pairing correlations, Phys. Rev. C 101, 054303 (2020).

[43] D. Vretenar, A. V. Afanasjev, G. A. Lalazissis, and P. Ring, Relativistic hartree-bogoliubov theory: Static and dynamic aspects of exotic nuclear structure, Phys. Rep. 409, 101 (2005).

[44] G. A. Lalazissis, S. Karatzikos, R. Fossion, D. P. Arteaga, A. V. Afanasjev, and P. Ring, The effective force nl3 revisited, Phys. Lett. B671, 36 (2009).

[45] T. Nikšić, D. Vretenar, and P. Ring, Relativistic nuclear energy density functionals: adjusting parameters to binding energies, Phys. Rev. C 78, 034318 (2008).

[46] G. A. Lalazissis, T. Nikšić, D. Vretenar, and P. Ring, New relativistic mean-field interaction with densitydependent meson-nucleon couplings, Phys. Rev. C 71, $024312(2005)$.

[47] X. Roca-Maza, X. Viñas, M. Centelles, P. Ring, and P. Schuck, Relativistic mean-field interaction with density-dependent meson-nucleon vertices based on microscopical calculations, Phys. Rev. C 84, 054309 (2011).

[48] P. W. Zhao, Z. P. Li, J. M. Yao, and J. Meng, New parametrization for the nuclear covariant energy density functional with a point-coupling interaction, Phys. Rev. C 82, 054319 (2010).

[49] Q. S. Zhang, Z. M. Niu, Z. P. Li, J. M. Yao, and J. Meng, Global dynamical correlation energies in covariant density functional theory: cranking approximation, Fron- tiers of Physics 9, 529 (2014).

[50] S. Raman, C. H. Malarkey, W. T. Milner, C. W. Nestor, Jr., and P. H. Stelson, At. Data Nucl. Data Tables 36 (1987).

[51] W. Nazarewicz and I. Ragnarsson, Nuclear deformations in Handbook on nuclear properties, Eds. D. N. Poenaru and W. Greiner (Claredon Press, Oxford, 1996), 80 .

[52] W. Bertozzi, J. Friar, J. Heisenberg, and J. W. Negele, Phys. Lett. B 41, 408 (1972).

[53] M. Nishimura and D. W. L. Sprung, Prog. Theor. Phys. 77, 781 (1987).

[54] S. E. Agbemava, A. V. Afanasjev, T. Nakatsukasa, and P. Ring, Covariant density functional theory: Reexamining the structure of superheavy nuclei, Phys. Rev. C 92, $054310(2015)$

[55] M. B. Tsang, J. R. Stone, F. Camera, P. Danielewicz, S. Gandolfi, K. Hebeler, C. J. Horowitz, J. Lee, W. G. ables.html.Lynch, Z. Kohley, R. Lemmon, P. Möller, T. Murakami, S. Riordan, X. Roca-Maza, F. Sammarruca, A. W. Steiner, I. Vidaña, and S. J. Yennello, Constraints on the symmetry energy and neutron skins from experiments and theory, Phys. Rev. C 86, 015803 (2012).

[56] X. Roca-Maza and N. Paar, Nuclear equation of state from ground and collective excited state properties of nuclei, Prog. Part. Nucl. Phys. 101, 96 (2018).

[57] A. Krasznahorkay, N. Paar, D. Vretenar, and M. N. Harakeh, Neutron-skin thickness of 208pb from the energy of the anti-analogue giant dipole resonance, Phys. Scripta T154, 014018 (2013).

[58] A. Krasznahorkay, M. Csatlós, L. Csige, T. K. Eriksen, F. Giacoppo, A. Görgen, T. W. Hagen, M. N. Harakeh, R. Julin, P. Koehler, N. Paar, S. Siem, L. Stuhl, T. Tornyi, and D. Vretenar, Neutron-skin thickness of 208pb, and symmetry-energy constraints from the study of the anti-analog giant dipole resonance, AIP Conf. Proc. 1491, 190 (2013).

[59] C. M. Tarbert, D. P. Watts, D. I. Glazier, P. Aguar, J. Ahrens, J. R. M. Annand, H. J. Arends, R. Beck, V. Bekrenev, B. Boillat, A. Braghieri, D. Branford, W. J. Briscoe, J. Brudvik, S. Cherepnya, R. Codling, E. J. Downie, K. Foehl, P. Grabmayr, R. Gregor, E. Heid, D. Hornidge, O. Jahn, V. L. Kashevarov, A. Knezevic, R. Kondratiev, M. Korolija, M. Kotulla, D. Krambrich, B. Krusche, M. Lang, V. Lisin, K. Livingston, S. Lugert, I. J. D. MacGregor, D. M. Manley, M. Martinez, J. C. McGeorge, D. Mekterovic, V. Metag, B. M. K. Nefkens, A. Nikolaev, R. Novotny, R. O. Owens, P. Pedroni, A. Polonski, S. N. Prakhov, J. W. Price, G. Rosner, M. Rost, T. Rostomyan, S. Schadmand, S. Schumann, D. Sober, A. Starostin, I. Supek, A. Thomas, M. Unverzagt, T. Walcher, and F. Zehr, The neutron skin of 208pb from coherent pion photoproduction, Phys. Rev. Lett. 112, 242502 (2014).

[60] C. J. Horowitz, Parity violating elastic electron scattering and coulomb distortions, Phys. Rev. C 57, 3430 (1998).

[61] D. Adhikari, H. Albataineh, D. Androic, K. Aniol, D. S. Armstrong, T. Averett, C. Ayerbe Gayoso, S. Barcus, V. Bellini, R. S. Beminiwattha, J. F. Benesch, H. Bhatt, D. Bhatta Pathak, D. Bhetuwal, B. Blaikie, Q. Campagna, A. Camsonne, G. D. Cates, Y. Chen, C. Clarke, J. C. Cornejo, S. Covrig Dusa, P. Datta, A. Deshpande, D. Dutta, C. Feldman, E. Fuchey, C. Gal, D. Gaskell, 
T. Gautam, M. Gericke, C. Ghosh, I. Halilovic, J.O. Hansen, F. Hauenstein, W. Henry, C. J. Horowitz, C. Jantzi, S. Jian, S. Johnston, D. C. Jones, B. Karki, S. Katugampola, C. Keppel, P. M. King, D. E. King, M. Knauss, K. S. Kumar, T. Kutz, N. Lashley-Colthirst, G. Leverick, H. Liu, N. Liyange, S. Malace, R. Mammei, J. Mammei, M. McCaughan, D. McNulty, D. Meekins, C. Metts, R. Michaels, M. M. Mondal, J. Napolitano, A. Narayan, D. Nikolaev, M. N. H. Rashad, V. Owen, C. Palatchi, J. Pan, B. Pandey, S. Park, K. D. Paschke, M. Petrusky, M. L. Pitt, S. Premathilake, A. J. R. Puckett, B. Quinn, R. Radloff, S. Rahman, A. Rathnayake, B. T. Reed, P. E. Reimer, R. Richards, S. Riordan, Y. Roblin, S. Seeds, A. Shahinyan, P. Souder, L. Tang, M. Thiel, Y. Tian, G. M. Urciuoli, E. W. Wertz, B. Wojtsekhowski, B. Yale, T. Ye, A. Yoon, A. Zec, W. Zhang, J. Zhang, and X. Zheng (PREX Collaboration), Accurate determination of the neutron skin thickness of ${ }^{208} \mathrm{~Pb}$ through parity-violation in electron scattering, Phys. Rev. Lett. 126, 172502 (2021).

[62] A. V. Afanasjev, S. E. Agbemava, D. Ray, and P. Ring, Neutron drip line: Single-particle degrees of freedom and pairing properties as sources of theoretical uncertainties, Phys. Rev. C 91, 014324 (2015).

[63] L. Bonneau, P. Quentin, and P. Möller, Global microscopic calculations of ground-state spins and parities for odd-mass nuclei, Phys. Rev. C 76, 024320 (2007).

[64] A. V. Afanasjev and S. Shawaqfeh, Deformed onequasiparticle states in covariant density functional theory, Phys. Lett. B 706, 177 (2011).

[65] J. Dobaczewski, A. V. Afanasjev, M. Bender, L. M. Robledo, and Y. Shi, Properties of nuclei in the nobelium region studied within the covariant, skyrme, and gogny energy density functionals, Nucl. Phys. A 944, 388 (2015).

[66] G. Coló, H. Sagawa, and P. F. Bortignon, Effect of particle-vibration coupling on single-particle states: A consistent study within the skyrme framework, Phys. Rev. C 82, 064307 (2010).

[67] E. V. Litvinova and A. V. Afanasjev, Dynamics of nuclear single-particle structure in covariant theory of particle-vibration coupling: From light to superheavy nuclei, Phys. Rev. C 84, 014305 (2011).

[68] A. V. Afanasjev and E. Litvinova, Impact of collective vibrations on quasiparticle states of open-shell odd-mass nuclei and possible interference with the tensor force, Phys. Rev. C 92, 044317 (2015).

[69] E. W. Otten, Nuclear radii and moments of unstable isotopes, in Treatise on Heavy Ion Science: Volume 8: Nuclei Far From Stability, edited by D. A. Bromley (Springer, Boston, MA, (1989), pp. 517638.

[70] W. Myers and K.-H. Schmidt, An update on dropletmodel charge distributions, Nucl. Phys. A410, 61 (1983).

[71] T. D. Goodacre, A. V. Afanasjev, A. E. Barzakh, L. Nies, B. A. Marsh, S. Sels, U. C. Perera, P. Ring, F. W. A. N. Andreyev, P. V. Duppen, N. A. Althubiti, B. Andel, D. Atanasov, R. S. Augusto, J. Billowes, K. Blaum, T. E. Cocolios, J. G. Cubiss, G. J. Farooq-Smith, D. V. F. V. N. Fedosseev, K. T. Flanagan, L. P. Ganey, L. Ghys, A. Gottberg, M. Huyse, S. Kreim, P. Kunz, D. Lunney, . K. M. Lynch, V. Manea, Y. M. Palenzuela, T. M. Medonca, P. L. Molkanov, M. Mougeot, J. P. Ramos, M. Rosenbusch, R. E. Rossel,
S. Rothe, L. Schweikhard, M. D. Seliverstov, P. Spagnoletti, C. V. Beveren, M. Veinhard, E. Verstraelen, A. Welker, K. Wendt, R. N. Wolf, A. Zadvornaya, and K. Zuber, submitted to Phys. Rev. C.

[72] J.-P. Delaroche, M. Girod, J. Libert, H. Goutte, S. Hilaire, S. Peru, N. Pillet, and G. F. Bertsch, Structure of even-even nuclei using a mapped collective hamiltonian and the d1s gogny interaction, Phys. Rev. C 81, 014303 (2010).

[73] www-phynu.cea.fr/science_en_ligne/carte_potentiels _microscopiques/carte_potentiel_nucleaire_eng.htm.

[74] M. Sharma, M. Nagarajan, and P. Ring, Rho meson coupling in the relativistic mean field theory and description of exotic nuclei, Phys. Lett. B 312, 377 (1993).

[75] S. E. Agbemava, A. V. Afanasjev, and A. Taninah, Propagation of statistical uncertainties in covariant density functional theory: Ground state observables and single-particle properties, Phys. Rev. C 99, 014318 (2019).

[76] P.-G. Reinhard, M. Rufa, J. Maruhn, W. Greiner, and J. Friedrich, Nuclear ground state properties in a relativistic meson field model, Z. Phys. A 323, 13 (1986).

[77] G. A. Lalazissis, J. König, and P. Ring, Phys. Rev. C 55, 540 (1997).

[78] A. Taninah, S. E. Agbemava, A. V. Afanasjev, and P. Ring, Parametric correlations in energy density functionals, Phys. Lett. B 800, 135065 (2020).

[79] L. Vermeeren, P. Lievensz, R. E. Silverans, U. Georg, M. Keimx, A. Klein, R. Neugart, M. Neuroth, F. Buchinger, and the ISOLDE Collaboration, The mean square nuclear charge radius of ${ }^{39}$ ca, J. Phys. G 22, 1517 (1996).

[80] C. J. Horowitz and J. Piekarewicz, Impact of spin-orbit currents on the electroweak skin of neutron-rich nuclei, Phys. Rev. C 86, 045503 (2012).

[81] H. Kurasawa and T. Suzuki, The $n$ th-order moment of the nuclear charge density and contribution from the neutrons, Prog. Th. Exp. Phys., 113D01 (2019).

[82] P.-G. Reinhard and W. Nazarewicz, Nuclear charge densities in spherical and deformed nuclei: Toward precise calculations of charge radii, Phys. Rev. C 103, 054310 (2021).

[83] J. L. Friar and J. W. Negele, Theoretical and experimental determination of nuclear charge distributions, Adv. Nucl. Phys, 219 (1975).

[84] T. Naito, G. Colò, H. Liang, and X. Roca-Maza, Second and fourth moments of the charge density and neutron-skin thickness of atomic nuclei, Phys. Rev. C 104, 024316 (2021).

[85] J. Zenihiro, T. Uesaka, H. Sagawa, and S. Yoshida, Proton density polarization of the doubly magic ${ }^{40}$ ca core in ${ }^{48} \mathrm{ca}$ and eos parameters, Prog. Theor. Exp. Phys. $023 D 05$ (2021).

[86] B. T. Reed, F. J. Fattoyev, C. J. Horowitz, and J. Piekarewicz, Implications of prex-2 on the equation of state of neutron-rich matter, Phys. Rev. Lett. 126, 172503 (2021).

[87] E. Ideguchi, D. G. Sarantites, W. Reviol, A. V. Afanasjev, M. Devlin, c. Baktash, R. V. F. Janssens, d. Rudolph, A. Axelsson, M. P. Carpenter, A. GalindoUribarri, D. R. LaFosse, T. Lauritsen, F. Lerma, C. J. Lister, P. Reiter, D. Seweryniak, M. Weiszflog, and J. N. Wilson, Superdeformation in the doubly magic nucleus ${ }_{20}^{40} \mathrm{ca}_{20}$, Phys. Rev. Lett. 87, 222501 (2001). 
[88] F. Barranco and R. A. Broglia, Correlation between mean square radii and zero-point motions of the surface in the ca isotopes, Phys. Lett. 151B, 90 (1985).

[89] S. Sels, T. Day Goodacre, B. A. Marsh, A. Pastore, W. Ryssens, Y. Tsunoda, N. Althubiti, B. Andel, A. N. Andreyev, D. Atanasov, A. E. Barzakh, M. Bender, J. Billowes, K. Blaum, T. E. Cocolios, J. G. Cubiss, J. Dobaczewski, G. J. Farooq-Smith, D. V. Fedorov, V. N. Fedosseev, K. T. Flanagan, L. P. Gaffney, L. Ghys, P.-H. Heenen, M. Huyse, S. Kreim, D. Lunney, K. M. Lynch, V. Manea, Y. Martinez Palenzuela, T. M. Medonca, P. L. Molkanov, T. Otsuka, J. P. Ramos, R. E. Rossel, S. Rothe, L. Schweikhard, M. D. Seliverstov, P. Spagnoletti, C. Van Beveren, P. Van Duppen, M. Veinhard, E. Verstraelen, A. Welker, K. Wendt, F. Wienholtz, R. N. Wolf, and A. Zadvornaya, Shape staggering of midshell mercury isotopes from in-source laser spectroscopy compared with density-functionaltheory and monte carlo shell-model calculations, Phys. Rev. C 99, 044306 (2019).

[90] M. Seliverstov, T. Cocolios, W. Dexters, A. Andreyev, S. Antalic, A. Barzakh, B. Bastin, J. Büscher, I. Darby, D. Fedorov, V. Fedoseyev, K. Flanagan, S. Franchoo, S. Fritzsche, G. Huber, M. Huyse, M. Keupers, U. Köster, Y. Kudryavtsev, B. Marsh, P. Molkanov, R. Page, A. Sjodin, I. Stefan, J. Van de Walle, P. Van Duppen, M. Venhart, and S. Zemlyanoy, Charge radii of odd-a 191211po isotopes, Phys. Lett. B 719, 362 (2013).

[91] D. A. Fink, T. E. Cocolios, A. N. Andreyev, S. Antalic, A. E. Barzakh, B. Bastin, D. V. Fedorov, V. N. Fedosseev, K. T. Flanagan, L. Ghys, A. Gottberg, M. Huyse, N. Imai, T. Kron, N. Lecesne, K. M. Lynch, B. A. Marsh, D. Pauwels, E. Rapisarda, S. D. Richter, R. E. Rossel, S. Rothe, M. D. Seliverstov, A. M. Sjödin, C. Van Beveren, P. Van Duppen, and K. D. A. Wendt, In-source laser spectroscopy with the laser ion source and trap: First direct study of the ground-state properties of ${ }^{217,219}$ Po, Phys. Rev. X 5, 011018 (2015).

[92] A. Voss, F. Buchinger, B. Cheal, J. E. Crawford, J. Dilling, M. Kortelainen, A. A. Kwiatkowski, A. Leary, C. D. P. Levy, F. Mooshammer, M. L. Ojeda, M. R. Pearson, T. J. Procter, and W. A. Tamimi, Nuclear moments and charge radii of neutron-deficient francium isotopes and isomers, Phys. Rev. C 91, 044307 (2015).

[93] J. G. Cubiss, A. E. Barzakh, M. D. Seliverstov, A. N. Andreyev, B. Andel, S. Antalic, P. Ascher, D. Atanasov, D. Beck, J. Bieroń, K. Blaum, C. Borgmann, M. Breitenfeldt, L. Capponi, T. E. Cocolios, T. Day Goodacre, X. Derkx, H. De Witte, J. Elseviers, D. V. Fedorov, V. N. Fedosseev, S. Fritzsche, L. P. Gaffney, S. George, L. Ghys, F. P. Heßberger, M. Huyse, N. Imai, Z. Kalaninová, D. Kisler, U. Köster, M. Kowalska, S. Kreim, J. F. W. Lane, V. Liberati, D. Lunney, K. M. Lynch, V. Manea, B. A. Marsh, S. Mitsuoka, P. L. Molkanov, Y. Nagame, D. Neidherr, K. Nishio, S. Ota, D. Pauwels, L. Popescu, D. Radulov, E. Rapisarda, J. P. Revill, M. Rosenbusch, R. E. Rossel, S. Rothe, K. Sandhu, L. Schweikhard, S. Sels, V. L. Truesdale, C. Van Beveren, P. Van den Bergh, Y. Wakabayashi, P. Van Duppen, K. D. A. Wendt, F. Wienholtz, B. W. Whitmore, G. L. Wilson, R. N. Wolf, and K. Zuber, Charge radii and electromagnetic moments of ${ }^{195--211}$ At, Phys. Rev. C 97, 054327 (2018).
[94] E. Verstraelen, A. Teigelhöfer, W. Ryssens, F. Ames, A. Barzakh, M. Bender, R. Ferrer, S. Goriely, P.-H. Heenen, M. Huyse, P. Kunz, J. Lassen, V. Manea, S. Raeder, and P. Van Duppen, Search for octupoledeformed actinium isotopes using resonance ionization spectroscopy, Phys. Rev. C 100, 044321 (2019).

[95] R. Ferrer, A. Barzakh, B. Bastin, R. Beerwerth, M. Block, P. Creemers, H. Grawe, R. de Groote, P. Delahaye, X. Fléchard, S. Franchoo, S. Fritzsche, L. Gaffney, L. Ghys, W. Gins, C. Granados, R. Heinke, L. Hijazi, M. Huyse, T. Kron, Y. Kudryavtsev, M. Laatiaoui, N. Lecesne, M. Loiselet, F. Lutton, I. Moore, Y. Mart'nez, E. Mogilevskiy, P. Naubereit, J. Piot, S. Raeder, S. Rothe, H. Savajols, S. Sels, V. Sonnenschein, J.-C. Thomas, E. Traykov, C. V. Beveren, P. V. den Bergh, P. V. Duppen, K. Wendt, and A. Zadvornaya, Towards high-resolution laser ionization spectroscopy of the heaviest elements in supersonic gas jet expansion, Nat. Commun. 8, 14520 (2017).

[96] S.Frauendorf and V.V.Pashkevich, On oblate-prolate transition in the ground state rotational band of light mercury isotopes, Phys. Lett. B 55, 365 (1975).

[97] M. Girod and P. Reinhard, A microscopic calculation of the collective potential and mass in hg-isotopes, Physics Letters B 117, 1 (1982).

[98] E. Litvinova, Quasiparticle-vibration coupling in a relativistic framework: Shell structure of $\mathrm{z}=120$ isotopes, Phys. Rev. C 85, 021303(R) (2012).

[99] R. Broglia, J. Damgaard, and A. Molinari, Particlevibration coupling in 209bi, Nucl. Phys. A 127, 429 (1969).

[100] I. Hamamoto, Particle-vibration coupling in the nuclei 209bi and 209pb, Nucl. Phys. A 126, 545 (1969).

[101] C. Mahaux, P. F. Bortignon, R. A. Broglia, and C. H. Dasso, Dynamics of the shell model, Phys. Rep. 120, 1 (1985).

[102] E. Litvinova and P. Ring, Covariant theory of particlevibrational coupling and its effect on the single-particle spectrum., Phys. Rev. C 73, 044328 (2006).

[103] P. Ring and E. Litvinova, Particle vibrational coupling in covariant density functional theory., Phys. At. Nucl. 72, 1285 (2009), translated from Yadernaya Fisika 72 (2009) No. 8, 1.

[104] R. R. Chasmasn, Incipient octupole deformation and parity doublets in the odd mass light actinides, Phys. Let. B 96.

[105] G. Leander and R. Sheline, Intrinsic reflection asymmetry in odd-a nuclei, Nuclear Physics A 413, 375 (1984).

[106] S. E. Agbemava, A. V. Afanasjev, and P. Ring, Octupole deformation in the ground states of even-even nuclei: a global analysis within the covariant density functional theory, Phys. Rev. C 93, 044304 (2016).

[107] Y. Cao, S. E. Agbemava, A. V. Afanasjev, W. Nazarewicz, and E. Olsen, Landscape of pearshaped even-even nuclei, Phys. Rev. C 102, 024311 (2020).

[108] J. Cocks, D. Hawcroft, N. Amzal, P. Butler, K. Cann, P. Greenlees, G. Jones, S. Asztalos, R. Clark, M. Deleplanque, R. Diamond, P. Fallon, I. Lee, A. Macchiavelli, R. MacLeod, F. Stephens, P. Jones, R. Julin, R. Broda, B. Fornal, J. Smith, T. Lauritsen, P. Bhattacharyya, and C. Zhang, Spectroscopy of rn, ra and th isotopes using multi-nucleon transfer reactions, Nucl. Phys. A 645, 61 (1999). 
[109] L. P. Gaffney, P. A. Butler, M. Scheck, A. B. Hayes, F. Wenander, M. Albers, B. Bastin, C. Bauer, A. Blazhev, S. Bönig, N. Bree, J. Cederkäll, T. Chupp, D. C. T. E. Cocolios, T. Davinson, H. D. Witte, J. Diriken, T. Grahn, A. Herzan, M. Huyse, D. G. Jenkins, D. T. Joss, N. K. J. Konki, M. Kowalczyk, T. Kröll, E. Kwan, R. Lutter, K. Moschner, P. Napiorkowski, J. Pakarinen, M. Pfeiffer, D. Radeck, P. Reiter, K. Reynders, S. V. Rigby, L. M. Robledo, M. Rudigier, S. Sambi, M. Seidlitz, B. Siebeck, T. Stora, P. Thoele, P. V. Duppen, M. J. Vermeulen, M. von Schmid, D. Voulot, N. Warr, K. Wimmer, K. Wrzosek-Lipska, C. Y. W., and M. Zielinska, Studies of pear-shaped nuclei using accelerated radioactive beams, Nature 497, 199 (2013).

[110] Y. R. Shimizu, J. D. Garrett, R. A. Broglia, M. Gallardo, and E. Vigezzi, Pairing fluctuations in rapidly rotating nuclei, Rev. Mod. Phys. 61, 131 (1989).

[111] P. Lievens, E. Arnold, W. Borchers, U. Georg, M. Keim, A. Klein, R. Neugart, L. Vermeeren, and R. E. Silverans, On the odd-even staggering of mean-square charge radii in the light krypton and strontium region, Europhys. Lett. 33, 11 (1996).

[112] A. V. Afanasjev and H. Abusara, Time-odd mean fields in covariant density functional theory: nonrotating systems, Phys. Rev. C 81, 014309 (2010).

[113] S. Teeti and A. V. Afanasjev, Global study of separable pairing interaction in covariant density functional theory, Phys. Rev. C 103, 034310 (2021).

[114] S. E. Agbemava, A. V. Afanasjev, D. Ray, and P. Ring, Assessing theoretical uncertainties in fission barriers of superheavy nuclei, Phys. Rev. C 95, 054324 (2017).

[115] E. N. E. van Dalen, C. Fuchs, and A. Faessler, Diracbrueckner-hartree-fock calculations for isospin asymmetric nuclear matter based on improved approximation schemes, Eur. Phys. J. A 31, 29 (2007).

[116] S. Wang, Q. Zhao, P. Ring, and J. Meng, Nuclear matter in relativistic brueckner-hartree-fock theory with bonn potential in the full dirac space, Phys. Rev. C 103, 054319 (2021), 2103.12960. 